\title{
NMR model of the entire membrane-interacting region of HIV-1 fusion protein and its perturbation of membrane morphology
}

\author{
Alessandro Piai ${ }^{1}$, Qingshan Fu ${ }^{1}$, Amanda K. Sharp ${ }^{2}$, Beatrice Bighi ${ }^{1}$, \\ Anne M. Brown ${ }^{2,3,4, *}$, and James J. Chou ${ }^{1, *}$ \\ ${ }^{1}$ Department of Biological Chemistry and Molecular Pharmacology, Harvard Medical School, \\ Boston, Massachusetts 02115, USA \\ 2 Interdisciplinary program in Genetics, Bioinformatics, and Computational Biology (GBCB), \\ Virginia Tech, Blacksburg, Virginia, 24061, USA \\ ${ }^{3}$ Research and Informatics, University Libraries, Virginia Tech, Blacksburg, Virginia 24061, USA \\ ${ }^{4}$ Department of Biochemistry, Virginia Tech, Blacksburg, Virginia 24061, USA
}




\section{Methods}

\section{Protein expression and purification}

The gp41 fragment encompassing the TMD and the full-length CT (residues 677-856) from a clade D HIV-1 isolate 92UG024.2 was synthesized by GenScript (Piscataway, NJ). The final NMR construct, designated TMD-CT, was created by fusing the gp41 fragment to the C-terminus of the Hisg-TrpLE expression sequence in pMM-LR6 vector with an added methionine in-between for subsequent cleavage during protein purification, as previously described ${ }^{1-}$ ${ }^{2}$. As previously done for the TMD-CTLP2 (ref ${ }^{1}$ ), unstructured residues 726-736 of the KS were deleted to reduce NMR signal overlap, and the two palmitoylation sites C764 and C837 were mutated to serine (C764S and C837S) to avoid non-physiological disulfide formation. Accidentally, an additional mutation A823G was introduced.

The TMD-CT construct was expressed by growing transformed E. coli strain BL21 (DE3) cells in M9 minimal media. Cultures were grown at $37^{\circ} \mathrm{C}$ until they reached an optical density of $\sim 0.6$ and were then cooled to $20^{\circ} \mathrm{C}$ before induction with $0.1 \mathrm{mM}$ isopropyl $\beta$-D-thiogalatopyranoside (IPTG). Protein was expressed at $20^{\circ} \mathrm{C}$ for $\sim 18-24 \mathrm{~h}$.

After growth, cells were harvested, suspended in a lysis buffer (50 mM Tris, pH 8.0, and $200 \mathrm{mM} \mathrm{NaCl}$ ), and lysed by sonication. Inclusion bodies were separated by centrifugation at $25,400 \times \mathrm{g}$ and suspended in a denaturing buffer (1\% Triton X-100, $6 \mathrm{M}$ guanidine hydrochloride, $50 \mathrm{mM}$ Tris, $\mathrm{pH} 8.0$, and $200 \mathrm{mM} \mathrm{NaCl}$ ). Inclusion bodies were homogenized using a glass tissue grinder, dissolved and centrifuged at $25,400 \times \mathrm{g}$. The fusion protein was bound to nickel affinity resin (Sigma-Aldrich), washed with $8 \mathrm{M}$ Urea and $\mathrm{dH}_{2} \mathrm{O}$, and finally eluted with $90 \%$ formic acid (FA). The gp41 construct was cleaved from the TrpLE by hydrolyzing the peptide bond at the C-terminus of the methionine using cyanogen bromide (CNBr) $(\sim 0.1 \mathrm{~g} / \mathrm{mL})$ in $90 \%$ FA for $1 \mathrm{~h}$. The reaction mixture was dialyzed (MWCO $3.5 \mathrm{kDa})$ to remove the excess of $\mathrm{CNBr}$ and $\mathrm{FA}$ and then lyophilized. The protein powder was dissolved in $90 \% \mathrm{FA}$ and purified by reverse-phase high-performance liquid chromatography (RP-HPLC) in a Zorbax SB-C3 column (Agilent Technologies, Santa Clara, CA) with a gradient from $95 \% \mathrm{dH}_{2} \mathrm{O}, 5 \%$ isopropanol (IPA), $0.1 \%$ trifluoroacetic acid (TFA) (buffer A) to 75\% IPA, 25\% acetonitrile, 0.1\% TFA (buffer B). Fractions containing the pure gp41 construct (confirmed by SDS-PAGE analysis) were collected and lyophilized.

\section{Protein reconstitution}

The lyophilized TMD-CT construct was dissolved in 1,1,1,3,3,3-hexafluoro-2-propanol (HFIP) and mixed with 9 mg of 1,2-Dimyristoyl-sn-glycero-3-phosphocholine (DMPC) (from Avanti Polar Lipids, Alabaster, AL or from FB Reagents, Boston, MA, if deuterated) and $27 \mathrm{mg}$ of 1,2-Dihexanoyl-sn-glycero-3-phosphocholine (DHPC) (from Avanti Polar Lipids Alabaster, AL or from FB Reagents Boston, MA, if deuterated). The mixture was dried under a nitrogen stream until a thin film was achieved and lyophilized overnight to completely remove any trace of residual organic solvent. The thin film was then dissolved in $3 \mathrm{~mL}$ of $8 \mathrm{M}$ Urea and dialyzed (MWCO $3.5 \mathrm{kDa}$ ) against $40 \mathrm{mM} \mathrm{MES} \mathrm{buffer,} \mathrm{pH}$ 6.7 to remove the denaturant. During and after the dialysis, additional DHPC was added to make up for the DHPC lost during the dialysis, adjusting the DMPC:DHPC ratio $(q)$ to $\sim 0.5$. The reconstituted protein was then concentrated using a Centricon (EMD Millipore, Billerica, MA) (MWCO $3.5 \mathrm{kDa}$ ) to 300-350 $\mu \mathrm{L}$. The buffer of the final NMR sample contained $40 \mathrm{mM}$ DMPC, $80 \mathrm{mM} \mathrm{DHPC}, 40 \mathrm{mM}$ MES, pH 6.7, 1\% NaN3, and 10\% (v/v) $\mathrm{D}_{2} \mathrm{O}$ (for the NMR lock). Finally, the bicelle $q$ of the NMR sample was quantified by signal integration of the DMPC and DHPC methyl peaks in the 1D ${ }^{1} \mathrm{H}$ NMR spectrum and adjusted to exactly 0.5 .

\section{NMR data acquisition and processing}

The NMR experiments were performed at (a) 14.1 T on a Bruker Avance III HD spectrometer operating at $600.13 \mathrm{MHz}{ }^{1} \mathrm{H}, 150.90 \mathrm{MHz}{ }^{13} \mathrm{C}$, and $60.81 \mathrm{MHz}{ }^{15} \mathrm{~N}$ frequencies; (b) 16.4 T on a Bruker Avance Neo spectrometer operating at $699.71 \mathrm{MHz}{ }^{1} \mathrm{H}, 175.94 \mathrm{MHz}{ }^{13} \mathrm{C}$, and $70.90 \mathrm{MHz}{ }^{15} \mathrm{~N}$ frequencies; (c) $18.8 \mathrm{~T}$ on a Bruker Avance III spectrometer operating at $800.28 \mathrm{MHz}{ }^{1} \mathrm{H}, 201.23 \mathrm{MHz}{ }^{13} \mathrm{C}$, and $81.09 \mathrm{MHz}{ }^{15} \mathrm{~N}$ frequencies; (d) $21.1 \mathrm{~T}$ on a Bruker Avance III spectrometer operating at $900.17 \mathrm{MHz}{ }^{1} \mathrm{H}, 226.35 \mathrm{MHz}{ }^{13} \mathrm{C}$, and $91.21 \mathrm{MHz}{ }^{15} \mathrm{~N}$ frequencies. All the spectrometers were equipped with a cryogenic probe. All the measurements were performed at $308 \mathrm{~K}$. The most relevant acquisition parameters of the experiments are reported in Table S2. 
The NMR data sets were processed with nmrPipe ${ }^{3}$ and the resulting NMR spectra were analyzed with Sparky (T. D. Goddard and D. G. Kneller, SPARKY 3, University of California, San Francisco) and XEASY4. Peak intensities were measured at peak local maxima using quadratic interpolation to identify peak centers. Origin (OriginLab, Northampton, MA) was used to fit the experimental data. For comparison purpose, the chemical shift assignments of the TMD ${ }^{5}$, MPER-TMD ${ }^{6}$ and TMD-CT ${ }^{L P 2}\left(\mathrm{ref}^{1}\right)$ were taken from the Biological Magnetic Resonance Bank (BMRB) entries 30090, 30503 and 30678, respectively.

\section{NMR resonance and NOE assignment}

Sequence specific assignment of TMD-CT backbone chemical shifts (BMRB accession code 30855) was accomplished using a set of 3D TROSY-enhanced triple resonance experiments (HNCA, $\mathrm{HNCO})^{7-8}$, recorded with a $\left({ }^{15} \mathrm{~N},{ }^{13} \mathrm{C}, 85 \%{ }^{2} \mathrm{H}\right.$ )labeled sample. In addition, a 3D ${ }^{15} \mathrm{~N}$-edited NOESY-TROSY-HSQC and a 3D ${ }^{15} \mathrm{~N}$-edited "double-TROSY" TROSYNOESY-TROSY-HSQC $\left(\tau_{m i x}=150 \mathrm{~ms}\right)$ spectra were recorded for completing the backbone resonance assignment. These NOESY experiments were performed on a sample reconstituted in bicelles with deuterated DMPC and DHPC acyl chains. Due to the large size and consequently unfavorable relaxation properties of the TMD-CT trimer, the sample required heavy deuteration and therefore the regular combination of $3 \mathrm{D}^{15} \mathrm{~N}$-edited NOESY-TROSY-HSQC and ${ }^{13} \mathrm{C}$-edited NOESY-HSQC spectra of a $\left({ }^{15} \mathrm{~N},{ }^{13} \mathrm{C}\right)$-labeled sample was not suitable for assigning protein aliphatic resonances. Instead, these two experiments were acquired using a $\left({ }^{15} \mathrm{~N}, 85 \%{ }^{2} \mathrm{H}\right)$ - and (ILV) ${ }^{13} \mathrm{C}$-labeled sample ( $\tau_{\text {mix }}=150$ and $200 \mathrm{~ms}$, respectively) reconstituted in bicelles with deuterated DMPC and DHPC acyl chains.

NOE-derived intra- and inter-chain distance restraints for the TMD-CT were obtained from the $3 \mathrm{D}^{15} \mathrm{~N}$-edited and ${ }^{13} \mathrm{C}$ edited NOESY spectra used for methyl resonance assignment above. Since the sample was not completely deuterated (85\%), some NOEs to protons other than methyl protons could also be assigned too. Some of the most important assigned NOEs are shown in Fig. S3.

\section{NMR-based membrane partition analyses}

The membrane partition of the TMD-CT was determined using the paramagnetic probe titration (PPT) method ${ }^{2,9}$. As previously shown, DMPC/DHPC bicelle with sufficiently large $q(\geq 0.5)$ allows direct use of measurable paramagnetic relaxation enhancement (PRE) to probe residue-specific immersion depth of the protein in the bilayer region of the bicelle. Two PPT analyses were performed: titrating the bicelle-reconstituted TMD-CT with (1) the soluble paramagnetic agent Gd-DOTA, and (2) the lipophilic paramagnetic agent 16-Doxyl-stearic acid (16-DSA). The titrants were taken from concentrated stock solutions (600 mM Gd-DOTA and $24 \mathrm{mM} 16-\mathrm{DSA}$ ) in the same buffer as that of the protein sample and were added in small aliquots (few $\mu \mathrm{L}$ per step) to minimize sample dilution. The PRE increase was monitored by recording a $2 \mathrm{D}^{1} \mathrm{H}-{ }^{15} \mathrm{~N}$ TROSY-HSQC spectrum at each of the titrant concentrations: 0 (reference), 1.0, 2.0, 4.0, 6.0, 8.0, 10.0, 15.0, and $20.0 \mathrm{mM}$ for Gd-DOTA; 0 (reference), 0.6, 1.2, 1.8, 2.4, 3.0 and $3.6 \mathrm{mM}$ for 16DSA. The residue-specific $P R E_{a m p}$, which is the amplitude of the PRE experienced by an amide proton in the protein, was determined by fitting the peak intensity decay as a function of [paramagnetic probe] to the following exponential decay equation:

$$
\frac{I}{I_{0}}=1-P R E_{\text {amp }}\left(1-e^{-\frac{[\text { paramagnetic probe }]}{\tau}}\right)
$$

where $I$ and $I_{0}$ are the peak intensities in the presence and absence of the paramagnetic probe, respectively, [paramagnetic probe] is the concentration of the paramagnetic agent (Gd-DOTA or 16-DSA), $\tau$ is the decay constant and $P R E_{a m p}$ is the PRE amplitude. The residue-specific $P R E_{a m p}$ (Table S3) were then used to determine the membrane partition of the protein $\left(r_{z}\right)$ by the sigmoidal fitting method ${ }^{2,9}$, in which the position of the TMD-CT trimer along the bilayer normal was fitted to satisfy Eq. S2:

$$
P R E_{a m p}=P R E_{a m p}^{\min }+\frac{\left(P R E_{a m p}^{\max }-P R E_{a m p}^{\min }\right)}{1+e^{\left(r_{Z}^{I}-\left|r_{Z}\right|\right) / S L O P E}}
$$


where $P R E_{a m p}^{\min }$ and $P R E_{a m p}^{\max }$ are the limits within which $P R E_{a m p}$ can vary for a particular protein system, $r_{Z}^{I}$ is the inflection point (the distance from the bilayer center at which $P R E_{a m p}$ is halfway between $P R E_{a m p}^{\min }$ and $P R E_{a m p}^{\max }$ ), and SLOPE is a parameter which reports the steepness of the curve at the inflection point.

\section{Measurement of inter-chain PRES}

Inter-chain PREs were measured for cross validation of the NOE-derived TMD-CT structure (Fig. S5) as previously described ${ }^{1-2}$. Three mixed samples were prepared by mixing, at $\sim 1: 1$ molar ratio, the $\left({ }^{15} \mathrm{~N}, 85 \%{ }^{2} \mathrm{H}\right)$-labeled TMDCT with one of the following unlabeled TMD-CT construct containing a specific Cys mutation/addition: (a) S764C (reintroducing the native cysteine); (b) S813C ; (c) C857 (Cys added to the C-terminus). The mutant proteins were prepared as in the "Protein expression and purification" section above. After reconstitution in bicelles under reducing conditions, DTT was removed from the samples by dialysis at low $\mathrm{pH}$ (6.2). The $\mathrm{pH}$ was then rapidly raised to 7.4 and $100 \mathrm{mM}$ MTSL (in DMSO) was added to a final ratio of 10:1 (MTSL to Cys-mutant TMD-CT) and allowed to react at room temperature overnight. Excess MTSL was removed by extensive dialysis (pH 6.7). The samples were then concentrated to $360 \mu \mathrm{L}$ for NMR measurements. During the entire sample preparation, MTSL-containing solutions were shielded from light. To quantify the PREs, defined as the ratio of the peak intensities in the paramagnetic $(I)$ and diamagnetic state $\left(I_{0}\right)$, a $2 \mathrm{D}^{1} \mathrm{H}-{ }^{15} \mathrm{~N}$ TROSY-HSQC spectrum was recorded before and after reducing the spin-label by addition of $20 \mathrm{mM}$ sodium ascorbate ( $\mathrm{pH} \mathrm{6.7).}$

\section{Plane restraints}

The PPT method ${ }^{1-2,9}$ was used to derive a set of plane restraints to aid structure calculation. Provided that the bicelle is sufficiently large $(q \geq 0.5)$, the PPT method allows to determine the projection of each amide proton $\left(r_{z}\right)$ along the protein $\mathrm{C}_{3}$ symmetry axis, which is also parallel to the bicelle normal and aligned to the Cartesian $\mathrm{Z}$ axis for convenience. Therefore, $r_{z}$ can be assigned as residue-specific plane restraint if the $P R E_{a m p}$ values are calibrated against a known structure with known membrane partition. Since the TMD-CT and the TMD show remarkable chemical shift and structure similarity for residues 679-710 (Fig. S1), this region was used to calibrate the $P R E_{a m p}$ values of the TMD-CT (from both Gd-DOTA and 16-DSA) against the previously established structure and membrane partition of the TMD ${ }^{5,10}$. In brief, we generated the $P R E_{a m p}$ vs. $r_{z}$ plot for residues $679-710$ using the residue-specific $P R E_{a m p}$ from the TMD-CT and the known $r_{z}$ from the known TMD structure. The data were then fitted to the sigmoidal function (Eq. S2) to yield parameterized Eq. S2 for Gd-DOTA and 16-DSA, which were then used to determine $r_{z}$ for the other residues of the TMD-CT not used for the calibration (711-856). Out of the calculated $r_{z}$, only those in the sensitive region of the sigmoidal curves were retained. Finally, $r_{z}$ derived from Gd-DOTA and 16DSA data sets were averaged and merged into one single data set, yielding the final set of plane restraints. The plane restraints used for the TMD-CT structure calculation included $r_{z}$ from the published TMD structure (residues 677710) and the newly calculated $r_{z}$ for residues 711-856, with an uncertainty of $\pm 1 \AA$.

Using the same procedure, plane restraints were also assigned for the MPER and included in the MPER-TMDCT model calculation. In this case, the previously published PRE ${ }_{a m p}$ values of the MPER-TMD (from Gd-DOTA titration) ${ }^{6}$ were calibrated against residues $695-708$ of the TMD as previously described ${ }^{1}$ and used to derive plane restraints for residues 660-694.

The data used to generate the plane restraints for the TMD-CT and MPER-TMD-CT (Table S4) are summarized in Fig. S4.

\section{Structure calculation}

NMR structures were calculated using XPLOR-NIH ${ }^{11}$. Since the TMD-CT (677-856) is an extension of the previously studied TMD-CT ${ }^{\text {LP2 }}$ (677-788) and both constructs exhibit almost identical chemical shift for residues 677-780 (Fig. S1b), we used the structure of the TMD-CT $T^{\mathrm{LP2}}\left(\operatorname{ref}^{1}\right)$ as a starting point for the new calculation. To implement this strategy, we first performed TALOS+ ${ }^{12}$ analysis using the assigned backbone chemical shift values $\left({ }^{15} \mathrm{~N},{ }^{13} \mathrm{C}^{\alpha}\right.$ and $\left.{ }^{13} \mathrm{C}^{\prime}\right)$, and then used the "GOOD" dihedral angles generated by TALOS+ to build a secondary structural 
model of the TMD-CT. Second, we assembled a trimer using the previously assigned NOE restraints for residues 677788 (from PDB accession code 6UJU). Finally, we included the newly assigned intra- and inter-chain restraints (most of them from residues 788-856) to complete the trimer of the TMD-CT. For each inter-chain restraint between two adjacent protomers, three identical distance restraints were assigned respectively to all pairs of neighboring protomers to satisfy the condition of $\mathrm{C}_{3}$ symmetry. The assembled trimer was then refined against the complete set of NOE restraints (intra- and inter-chain) and dihedral angles using a simulated annealing (SA) protocol in which the temperature was lowered from 1000 to $200 \mathrm{~K}$ in steps of $50 \mathrm{~K}$. The NOE restraints were enforced by flat-well harmonic potentials, with the force constant ramped from 2 to $40 \mathrm{kcal} / \mathrm{mol} \AA^{-2}$ during annealing. Backbone dihedral angle restraints were enforced by flat-well ( \pm the corresponding uncertainties from TALOS+) harmonic potential with force constant ramped from 10 to $1000 \mathrm{kcal} / \mathrm{mol} \mathrm{rad}^{-2}$. The plane restraints were fixed in space and enforced by flatwell ( $\pm 1 \AA$ ) harmonic potentials, with force constant ramped from 1 to $5 \mathrm{kcal} / \mathrm{mol} \AA^{-2}$ during annealing. A total of 150 structures were calculated, and the 15 lowest energy structures were selected as the final structural ensemble (PDB accession code 7LOH) (Fig. S6a and Table S5).

The model of the MPER-TMD-CT was generated in a similar manner. The matching resonances of the TM core (residues 685-700) in the MPER-TMD (660-710), TMD (677-716), TMD-CT ${ }^{\text {LLP2 }}$ (677-788) and TMD-CT (677-856) (Fig. S1) allowed to merge the four structures at the TM core to generate a MPER-TMD-CT starting model for further refinement. NOEs and backbone dihedral angle restraints for the MPER, TMD and CT ${ }^{\text {LLP2 }}$ (residues 660-693, 694-710 and 711-785 respectively) were taken from previously published studies ${ }^{1,5-6}$, while those for the CT ${ }^{\text {LP3-LLP1 }}$ domain (789-856) were taken from the current study. Plane restraints from the three regions (MPER, TMD and CT) were also applied. The model was refined using identical parameters and potentials as those used for the TMD-CT (see above). A total of 150 structures were calculated, and the 15 lowest energy structures were selected as the final structural ensemble (PDB accession code 7LOI) (Fig. S6b and Table S6).

\section{Molecular dynamics simulations of gp41 MPER-TMD-CT in an asymmetric membrane}

Molecular dynamics (MD) simulations were built using the gp41 MPER-TMD-CT NMR model with the CHARMM-GUI membrane builder ${ }^{13}$ and simulated using GROMACS v2020.3 software package ${ }^{14-15}$. Lipids distribution per leaflet of the membrane is displayed in Table S1 and mimics previous simulation work ${ }^{16}$ and the native HIV membrane ${ }^{17}$. The CHARMM36m force field ${ }^{18}$ was used with the TIP3P water model ${ }^{19}$ and $0.150 \mathrm{M} \mathrm{KCl}$ solvent with charge neutralizing ions $^{20}$. Minimization was performed using the steepest decent integrator algorithm and a tolerance of $1000 \mathrm{~kJ} \mathrm{~mol}^{-}$ ${ }^{1} \mathrm{~nm}^{-1}$. Three replicates were generated at the beginning of equilibration with the MD leap-frog algorithm integrator. Equilibration was competed in six phases, first phase applied each replicate with different first phase random velocities using Berendsen thermostat temperature coupling method ${ }^{21}$, second phase as a continuation of phase one, third phase added in the Berendsen thermostat pressure coupling ${ }^{21}$ and applied the semi-isotropic isotropy pressure coupling method, while the fourth phase, fifth phase, and sixth phase is a continuation of the third while slowly decreasing the position restraints for the system. Production simulations were performed at $310 \mathrm{~K}$ and $1 \mathrm{bar}$, and used the Nose-Hoover temperature coupling method ${ }^{22-23}$, the Parrinello-Rahman pressure coupling method ${ }^{23-}$ 24 , the fourth-order P-LINCS constraint algorithm ${ }^{25}$, and the Verlet cutoff-scheme. All position restrains were removed in production simulations. Electrostatics were calculated using the Fast smooth Particle-Mesh Ewald algorithm ${ }^{26-27}$ with a coulomb cutoff of $1.2 \mathrm{~nm}$ and $0.16 \mathrm{~nm}$ Fourier grid space. Van der Waals were calculated using the Lennard Jones equation cut-off that switches forces to zero from 1.0-1.2 nm using a force-switch method. All three dimensions used periodic boundary conditions. Systems were simulated to $1 \mu \mathrm{s}$ (1000 ns), with three replicates, for a total of 3000 ns of simulation time. Analysis of protein structure and membrane stability were completed over the last 100 ns of the simulation based off determined convergence from root mean-square deviation (RMSD) analysis and RMSD clustering analysis. Structural visualizations were performed in PyMOL (The PyMOL Molecular Graphics System, Version 2.4.0; Schrödinger, LLC). Parameter files and in-house scripts available on our Open Science Framework page (https://osf.io/82n73/). 
Table S1. Lipid composition per leaflet with gp41 MPER-TMD-CT trimer inserted in the membrane.

\begin{tabular}{lcc}
\hline Lipid type & Exofacial leaflet number & Cytofacial leaflet number \\
\hline Cholesterol & 271 & 195 \\
POPC & 99 & 0 \\
POPE & 0 & 40 \\
SOPS & 0 & 80 \\
SDPE & 0 & 135 \\
PSM & 204 & 0 \\
\hline
\end{tabular}

POPC (1-palmitoyl-2-oleoyl-sn-phosphatidylcholine)

POPE (1-palmitoyl-2-oleoyl-sn-phosphatidylethanolamine)

SOPS (stearoyl-oleoyl-phosphatidylserine)

SDPE (1-Stearoyl-2-docosahexaenoyl-sn-glycerophosphoethanolamine)

PSM (Palmitoylsphingomyelin) 
Table S2. NMR acquisition parameters.

\begin{tabular}{|c|c|c|c|c|c|}
\hline Type of experiment & $\begin{array}{c}\text { Spectral widths and } \\
\text { chemical shift evolution } \\
\text { times }\end{array}$ & $\begin{array}{l}\text { \# of } \\
\text { scans }\end{array}$ & $\begin{array}{c}\text { Inter-scan } \\
\text { delay (s) }\end{array}$ & $\begin{array}{l}\text { Duration of } \\
\text { the } \\
\text { experiment }\end{array}$ & Notes \\
\hline \multicolumn{6}{|c|}{ Backbone, side-chain and NOE assignment } \\
\hline \multicolumn{6}{|c|}{$\sim 1.2 \mathrm{mM}\left({ }^{15} \mathrm{~N},{ }^{13} \mathrm{C}, 85 \%{ }^{2} \mathrm{H}\right)$-labeled TMD-CT; magnetic field: $21.1 \mathrm{~T}$} \\
\hline $2 \mathrm{D}^{1} \mathrm{H}-{ }^{15} \mathrm{~N}$ TROSY HSQC & $\begin{array}{c}10900 \mathrm{~Hz}, 94.0 \mathrm{~ms}\left({ }^{1} \mathrm{H}^{\mathrm{N}}\right) \\
1950 \mathrm{~Hz}, 76.9 \mathrm{~ms}\left({ }^{15} \mathrm{~N}\right)\end{array}$ & 24 & 1.2 & $3 \mathrm{~h}$ & \\
\hline 3D TROSY HNCO & $\begin{array}{c}10900 \mathrm{~Hz}, 94.0 \mathrm{~ms}\left({ }^{1} \mathrm{H}^{\mathrm{N}}\right) \\
1950 \mathrm{~Hz}, 40.0 \mathrm{~ms}\left({ }^{15} \mathrm{~N}\right) \\
1900 \mathrm{~Hz}, 7.9 \mathrm{~ms}\left({ }^{13} \mathrm{C}^{\prime}\right)\end{array}$ & 24 & 1.2 & $1 \mathrm{~d} 21 \mathrm{~h}$ & \\
\hline 3D TROSY HNCA & $\begin{array}{c}10900 \mathrm{~Hz}, 94.0 \mathrm{~ms}\left({ }^{1} \mathrm{H}^{\mathrm{N}}\right) \\
1950 \mathrm{~Hz}, 40.0 \mathrm{~ms}\left({ }^{15} \mathrm{~N}\right) \\
6000 \mathrm{~Hz}, 6.0 \mathrm{~ms}\left({ }^{13} \mathrm{C}^{\alpha}\right)\end{array}$ & 36 & 1.2 & $6 \mathrm{~d} 16 \mathrm{~h}$ & \\
\hline \multicolumn{6}{|c|}{$\sim 1.0 \mathrm{mM}\left({ }^{15} \mathrm{~N}, 85 \%{ }^{2} \mathrm{H}\right)$ - and $(\mathrm{ILV}){ }^{13} \mathrm{C}$-labeled TMD-CT; magnetic field: $21.1 \mathrm{~T}$} \\
\hline $2 \mathrm{D}^{1} \mathrm{H}-{ }^{15} \mathrm{~N}$ TROSY HSQC & $\begin{array}{c}10900 \mathrm{~Hz}, 94.0 \mathrm{~ms}\left({ }^{1} \mathrm{H}^{\mathrm{N}}\right) \\
1950 \mathrm{~Hz}, 76.9 \mathrm{~ms}\left({ }^{15} \mathrm{~N}\right)\end{array}$ & 24 & 1.2 & $3 \mathrm{~h}$ & \\
\hline $2 \mathrm{D}^{1} \mathrm{H}-{ }^{13} \mathrm{CHSQC}$ & $\begin{array}{c}10900 \mathrm{~Hz}, 47.0 \mathrm{~ms}\left({ }^{1} \mathrm{H}^{\mathrm{N}}\right) \\
4000 \mathrm{~Hz}, 56.0 \mathrm{~ms}\left({ }^{13} \mathrm{C}\right)\end{array}$ & 8 & 1.2 & $1 \mathrm{~h}$ & \\
\hline $\begin{array}{l}3 \mathrm{D}^{15} \mathrm{~N} \text {-edited NOESY- } \\
\text { TROSY-HSQC }\end{array}$ & $\begin{array}{c}10900 \mathrm{~Hz}, 94.0 \mathrm{~ms}\left({ }^{1} \mathrm{H}^{\mathrm{N}}\right) \\
1950 \mathrm{~Hz}, 30.3 \mathrm{~ms}\left({ }^{15} \mathrm{~N}\right) \\
11200 \mathrm{~Hz}, 8.6 \mathrm{~ms}\left({ }^{1} \mathrm{H}\right)\end{array}$ & 20 & 1.2 & $8 \mathrm{~d} 2 \mathrm{~h}$ & $\tau_{\text {mix }}=150 \mathrm{~ms}$ \\
\hline $3 \mathrm{D}^{13} \mathrm{C}$-edited NOESY & $\begin{array}{c}10900 \mathrm{~Hz}, 47.0 \mathrm{~ms}\left({ }^{1} \mathrm{H}^{\mathrm{N}}\right) \\
4000 \mathrm{~Hz}, 16.0 \mathrm{~ms}\left({ }^{13} \mathrm{C}\right) \\
11200 \mathrm{~Hz}, 8.6 \mathrm{~ms}\left({ }^{1} \mathrm{H}\right)\end{array}$ & 12 & 1.2 & $5 \mathrm{~d} 7 \mathrm{~h}$ & $\tau_{\text {mix }}=200 \mathrm{~ms}$ \\
\hline $\begin{array}{l}3 D^{15} \mathrm{~N} \text {-edited TROSY- } \\
\text { NOESY-TROSY-HSQC }\end{array}$ & $\begin{array}{c}10900 \mathrm{~Hz}, 94.0 \mathrm{~ms}\left({ }^{1} \mathrm{H}^{\mathrm{N}}\right) \\
1950 \mathrm{~Hz}, 20.5 \mathrm{~ms}\left({ }^{15} \mathrm{~N}\right) \\
1950 \mathrm{~Hz}, 20.5 \mathrm{~ms}\left({ }^{15} \mathrm{~N}\right)\end{array}$ & 116 & 1.2 & $13 \mathrm{~d} 9 \mathrm{~h}$ & $\tau_{\text {mix }}=150 \mathrm{~ms}$ \\
\hline \multicolumn{6}{|c|}{ PPT } \\
\hline \multicolumn{6}{|c|}{$\sim 0.7 \mathrm{mM}\left({ }^{15} \mathrm{~N}, 85 \%{ }^{2} \mathrm{H}\right)$-labeled TMD-CT; magnetic field: $14.1 \mathrm{~T}$} \\
\hline $2 \mathrm{D}^{1} \mathrm{H}-{ }^{15} \mathrm{~N}$ TROSY HSQC & $\begin{array}{c}9600 \mathrm{~Hz}, 106.0 \mathrm{~ms}\left({ }^{1} \mathrm{H}^{\mathrm{N}}\right) \\
1300 \mathrm{~Hz}, 76.9 \mathrm{~ms}\left({ }^{15} \mathrm{~N}\right)\end{array}$ & 48 & 3.5 & $\begin{array}{l}10 \mathrm{~h} \text { (each } \\
\text { spectrum) }\end{array}$ & $\begin{array}{c}\text { [Gd-DOTA] }= \\
0,1.0,2.0 \\
4.0,6.0,8.0 \\
10.0,15.0 \\
20.0 \mathrm{mM}\end{array}$ \\
\hline \multicolumn{6}{|c|}{$\sim 0.7 \mathrm{mM}\left({ }^{15} \mathrm{~N}, 85 \%{ }^{2} \mathrm{H}\right)$-labeled TMD-CT; magnetic field: $14.1 \mathrm{~T}$} \\
\hline $2 \mathrm{D}^{1} \mathrm{H}-{ }^{15} \mathrm{~N}$ TROSY HSQC & $\begin{array}{c}9600 \mathrm{~Hz}, 106.0 \mathrm{~ms}\left({ }^{1} \mathrm{H}^{\mathrm{N}}\right) \\
1300 \mathrm{~Hz}, 76.9 \mathrm{~ms}\left({ }^{15} \mathrm{~N}\right)\end{array}$ & 48 & 3.5 & $\begin{array}{l}10 \mathrm{~h} \text { (each } \\
\text { spectrum) }\end{array}$ & $\begin{array}{c}{[16-\mathrm{DSA}]=0} \\
0.6,1.2,1.8 \\
2.4,3.0,3.6 \\
\mathrm{mM}\end{array}$ \\
\hline
\end{tabular}


Table S2 (continued)

\begin{tabular}{|c|c|c|c|c|c|}
\hline Type of experiment & $\begin{array}{c}\text { Spectral widths and } \\
\text { chemical shift evolution } \\
\text { times }\end{array}$ & $\begin{array}{l}\text { \# of } \\
\text { scans }\end{array}$ & $\begin{array}{c}\text { Inter-scan } \\
\text { delay (s) }\end{array}$ & $\begin{array}{l}\begin{array}{c}\text { Duration of } \\
\text { the } \\
\text { experiment }\end{array} \\
\end{array}$ & Notes \\
\hline \multicolumn{6}{|c|}{ Inter-protomer PREs } \\
\hline \multicolumn{6}{|c|}{$\begin{array}{c}\sim 0.45 \mathrm{mM}\left({ }^{15} \mathrm{~N}, 85 \%{ }^{2} \mathrm{H}\right) \text {-labeled TMD-CT mixed with } \sim 0.45 \mathrm{mM} \text { unlabeled TMD-CT S764C ( 1:1); } \\
\text { magnetic field: } 16.4 \mathrm{~T}\end{array}$} \\
\hline $2 \mathrm{D}^{1} \mathrm{H}-{ }^{15} \mathrm{~N}$ TROSY HSQC & $\begin{array}{c}12500 \mathrm{~Hz}, 82.0 \mathrm{~ms}\left({ }^{1} \mathrm{H}^{\mathrm{N}}\right) \\
1520 \mathrm{~Hz}, 98.7 \mathrm{~ms}\left({ }^{15} \mathrm{~N}\right)\end{array}$ & 136 & 3.5 & $\begin{array}{c}1 \mathrm{~d} 18 \mathrm{~h} \\
\text { (each } \\
\text { spectrum) }\end{array}$ & $\begin{array}{c}\text { [Sodium } \\
\text { ascorbate] = } \\
0,20.0 \mathrm{mM}\end{array}$ \\
\hline \multicolumn{6}{|c|}{$\begin{array}{c}\sim 0.45 \mathrm{mM}\left({ }^{15} \mathrm{~N}, 85 \%{ }^{2} \mathrm{H}\right) \text {-labeled TMD-CT mixed with } \sim 0.45 \mathrm{mM} \text { unlabeled TMD-CT S813C ( 1:1); } \\
\text { magnetic field: } 16.4 \mathrm{~T}\end{array}$} \\
\hline $2 \mathrm{D}^{1} \mathrm{H}-{ }^{15} \mathrm{~N}$ TROSY HSQC & $\begin{array}{c}12500 \mathrm{~Hz}, 82.0 \mathrm{~ms}\left({ }^{1} \mathrm{H}^{\mathrm{N}}\right) \\
1520 \mathrm{~Hz}, 98.7 \mathrm{~ms}\left({ }^{15} \mathrm{~N}\right)\end{array}$ & 136 & 3.5 & $\begin{array}{c}1 \mathrm{~d} 18 \mathrm{~h} \\
\text { (each } \\
\text { spectrum) }\end{array}$ & $\begin{array}{c}\text { [Sodium } \\
\text { ascorbate] = } \\
0,20.0 \mathrm{mM}\end{array}$ \\
\hline \multicolumn{6}{|c|}{$\begin{array}{l}\sim 0.4 \mathrm{mM}\left({ }^{15} \mathrm{~N}, 85 \%{ }^{2} \mathrm{H}\right) \text {-labeled TMD-CT mixed with } \sim 0.4 \mathrm{mM} \text { unlabeled TMD-CT C857 ( 1:1); } \\
\text { magnetic field: } 14.1 \mathrm{~T}\end{array}$} \\
\hline $2 \mathrm{D}^{1} \mathrm{H}-{ }^{15} \mathrm{~N}$ TROSY HSQC & $\begin{array}{c}9600 \mathrm{~Hz}, 106.0 \mathrm{~ms}\left({ }^{1} \mathrm{H}^{\mathrm{N}}\right) \\
1300 \mathrm{~Hz}, 98.5 \mathrm{~ms}\left({ }^{15} \mathrm{~N}\right)\end{array}$ & 160 & 3.5 & $\begin{array}{l}1 \mathrm{~d} 18 \mathrm{~h} \\
\text { (each } \\
\text { spectrum) }\end{array}$ & $\begin{array}{c}\text { [Sodium } \\
\text { ascorbate] = } \\
0,20.0 \mathrm{mM}\end{array}$ \\
\hline \multicolumn{6}{|c|}{$\sim 0.6 \mathrm{mM}\left({ }^{15} \mathrm{~N},{ }^{13} \mathrm{C}, 85 \%{ }^{2} \mathrm{H}\right)$-labeled TMD-CT $\mathrm{CT}^{\mathrm{LL} 2}$; magnetic field: $18.8 \mathrm{~T}$} \\
\hline $2 \mathrm{D}^{1} \mathrm{H}-{ }^{15} \mathrm{~N}$ TROSY HSQC & $\begin{array}{c}11150 \mathrm{~Hz}, 92.0 \mathrm{~ms}\left({ }^{1} \mathrm{H}^{\mathrm{N}}\right) \\
1900 \mathrm{~Hz}, 79.0 \mathrm{~ms}\left({ }^{15} \mathrm{~N}\right)\end{array}$ & 32 & 1.2 & $4 \mathrm{~h}$ & \\
\hline 3D J $\mathrm{J}_{\mathrm{CH}}$-modulated NOESY & $\begin{array}{c}11500 \mathrm{~Hz}, 92.0 \mathrm{~ms}\left({ }^{1} \mathrm{H}^{\mathrm{N}}\right) \\
1900 \mathrm{~Hz}, 24.2 \mathrm{~ms}\left({ }^{15} \mathrm{~N}\right) \\
9600 \mathrm{~Hz}, 7.5 \mathrm{~ms}\left({ }^{1} \mathrm{H}\right)\end{array}$ & 20 & 1.2 & $9 \mathrm{~d} 4 \mathrm{~h}$ & $\tau_{\text {mix }}=100 \mathrm{~ms}$ \\
\hline
\end{tabular}


Table S3. Residue specific $P R E_{a m p}$ of TMD-CT in $q=0.5$ bicelles. The values were determined by fitting $I / I_{0}$ vs. [paramagnetic agent] to Eq. S1. PRE $E_{a m p}$ of non-proline residues not shown were discarded because of signal overlap or poor data fitting.

\begin{tabular}{|c|c|c|c|c|c|}
\hline Residue & $\begin{array}{c}\text { PRE } E_{a m p} \\
\text { (Gd-DOTA) }\end{array}$ & $\begin{array}{c}\text { PRE } E_{a m p} \\
\text { (16-DSA) }\end{array}$ & Residue & $\begin{array}{c}\text { PRE } E_{a m p} \\
\text { (Gd-DOTA) }\end{array}$ & $\begin{array}{c}\text { PRE } E_{a m p} \\
\text { (16-DSA) }\end{array}$ \\
\hline 679 & $0.895 \pm 0.031$ & $0.898 \pm 0.026$ & 738 & $0.949 \pm 0.046$ & $-2.272 \pm 0.062(*)$ \\
\hline 681 & $0.963 \pm 0.024$ & $0.920 \pm 0.012$ & 739 & $0.899 \pm 0.043$ & $-0.344 \pm 0.018(*)$ \\
\hline 684 & $0.868 \pm 0.027$ & $0.959 \pm 0.023$ & 742 & $0.901 \pm 0.023$ & $-0.0076 \pm 0.0028\left(^{*}\right)$ \\
\hline 685 & - & $0.954 \pm 0.031$ & 744 & $0.890 \pm 0.033$ & $0.445 \pm 0.027(*)$ \\
\hline 686 & $0.767 \pm 0.060$ & $0.9679 \pm 0.0082$ & 745 & $0.964 \pm 0.023$ & $0.767 \pm 0.040(*)$ \\
\hline 688 & $0.715 \pm 0.069$ & $0.991 \pm 0.018$ & 747 & $0.903 \pm 0.033$ & $0.893 \pm 0.023$ \\
\hline 690 & $0.671 \pm 0.083$ & $0.9899 \pm 0.0097$ & 748 & $0.940 \pm 0.027$ & $0.9479 \pm 0.0088$ \\
\hline 691 & $0.643 \pm 0.022$ & $0.982 \pm 0.021$ & 750 & $0.901 \pm 0.053$ & $0.945 \pm 0.032$ \\
\hline 693 & - & $0.979 \pm 0.018$ & 753 & $0.941 \pm 0.022$ & $0.963 \pm 0.014$ \\
\hline 694 & $0.648 \pm 0.0 .067$ & $0.982 \pm 0.014$ & 754 & $0.900 \pm 0.016$ & $0.924 \pm 0.015$ \\
\hline 695 & $0.656 \pm 0.060$ & $0.982 \pm 0.015$ & 755 & $0.914 \pm 0.043$ & $0.948 \pm 0.020$ \\
\hline 696 & $0.635 \pm 0.079$ & $0.984 \pm 0.015$ & 756 & $0.874 \pm 0.064$ & $0.951 \pm 0.011$ \\
\hline 698 & $0.688 \pm 0.069$ & $0.989 \pm 0.010$ & 758 & $0.880 \pm 0.049$ & $0.944 \pm 0.019$ \\
\hline 699 & $0.726 \pm 0.077$ & $0.9853 \pm 0.0029$ & 762 & $0.894 \pm 0.030$ & $0.933 \pm 0.019$ \\
\hline 700 & $0.729 \pm 0.056$ & $0.9903 \pm 0.0073$ & 763 & $0.853 \pm 0.045$ & - \\
\hline 702 & $0.799 \pm 0.075$ & $0.984 \pm 0.012$ & 770 & $0.927 \pm 0.042$ & $0.9653 \pm 0.0056$ \\
\hline 703 & $0.810 \pm 0.058$ & $0.975 \pm 0.012$ & 771 & $0.873 \pm 0.037$ & $0.919 \pm 0.043$ \\
\hline 704 & $0.868 \pm 0.066$ & $0.960 \pm 0.013$ & 772 & $0.885 \pm 0.028$ & $0.9784 \pm 0.0077$ \\
\hline 705 & - & $0.948 \pm 0.030$ & 774 & $0.859 \pm 0.044$ & $0.934 \pm 0.025$ \\
\hline 706 & $0.877 \pm 0.041$ & $0.897 \pm 0.024$ & 775 & - & $0.9739 \pm 0.0093$ \\
\hline 707 & $0.889 \pm 0.052$ & $0.896 \pm 0.013$ & 776 & $0.878 \pm 0.038$ & $0.934 \pm 0.020$ \\
\hline 710 & $0.934 \pm 0.033$ & $0.884 \pm 0.027$ & 779 & $0.836 \pm 0.048$ & $0.982 \pm 0.011$ \\
\hline 711 & $0.970 \pm 0.017$ & $0.856 \pm 0.038$ & 782 & $0.861 \pm 0.050$ & $0.981 \pm 0.010$ \\
\hline 712 & $0.918 \pm 0.034$ & $0.885 \pm 0.022$ & 785 & $0.933 \pm 0.085$ & $0.960 \pm 0.021$ \\
\hline 713 & $0.943 \pm 0.018$ & $0.871 \pm 0.034$ & 786 & $0.958 \pm 0.047$ & $0.961 \pm 0.022$ \\
\hline 714 & $0.910 \pm 0.021$ & - & 788 & $0.810 \pm 0.051$ & $0.929 \pm 0.014$ \\
\hline 715 & $0.962 \pm 0.021$ & $0.929 \pm 0.016$ & 789 & - & $0.901 \pm 0.022$ \\
\hline 716 & $0.928 \pm 0.030$ & $0.890 \pm 0.028$ & 790 & - & $0.912 \pm 0.052$ \\
\hline 717 & $0.959 \pm 0.029$ & $0.824 \pm 0.033(*)$ & 791 & $0.816 \pm 0.073$ & $0.942 \pm 0.035$ \\
\hline 719 & $0.953 \pm 0.013$ & $0.746 \pm 0.076(*)$ & 792 & $0.858 \pm 0.095$ & $0.915 \pm 0.038$ \\
\hline 721 & $0.974 \pm 0.024$ & $0.755 \pm 0.076(*)$ & 793 & $0.961 \pm 0.048$ & $0.974 \pm 0.019$ \\
\hline 723 & $0.970 \pm 0.022$ & $0.647 \pm 0.099(*)$ & 794 & $0.895 \pm 0.024$ & $0.973 \pm 0.033$ \\
\hline 725 & $0.929 \pm 0.037$ & $-0.060 \pm 0.087\left(^{*}\right)$ & 795 & $0.819 \pm 0.062$ & $0.908 \pm 0.056$ \\
\hline 737 & $0.931 \pm 0.036$ & $-2.445 \pm 0.092\left(^{*}\right)$ & 796 & $0.799 \pm 0.040$ & $0.933 \pm 0.034$ \\
\hline
\end{tabular}


Table S3 (continued)

\begin{tabular}{|c|c|c|c|c|c|}
\hline Residue & $\begin{array}{c}P_{R E_{a m p}} \\
\text { (Gd-DOTA) }\end{array}$ & $\begin{array}{c}P_{R E_{a m p}} \\
(16-D S A)\end{array}$ & Residue & $\begin{array}{c}\text { PRE }_{a m p} \\
\text { (Gd-DOTA) }\end{array}$ & $\begin{array}{c}P_{R E_{a m p}} \\
(16-D S A)\end{array}$ \\
\hline 797 & $0.919 \pm 0.073$ & $0.977 \pm 0.024$ & 826 & $0.936 \pm 0.077$ & $0.976 \pm 0.040$ \\
\hline 799 & $0863 . \pm 0.053$ & $0.973 \pm 0.025$ & 829 & $0.941 \pm 0.088$ & $0.943 \pm 0.037$ \\
\hline 800 & $0.892 \pm 0.045$ & $0.983 \pm 0.014$ & 832 & $0.894 \pm 0.060$ & $0.921 \pm 0.036$ \\
\hline 801 & $0.956 \pm 0.076$ & $0.896 \pm 0.036$ & 833 & $0.863 \pm 0.073$ & $0.957 \pm 0.027$ \\
\hline 802 & $0.799 \pm 0.061$ & $0.922 \pm 0.057$ & 834 & $0.908 \pm 0.054$ & $0.916 \pm 0.034$ \\
\hline 803 & $0.853 \pm 0.062$ & $0.974 \pm 0.024$ & 835 & $0.930 \pm 0.035$ & $0.893 \pm 0.046$ \\
\hline 804 & $0.879 \pm 0.037$ & $0.985 \pm 0.032$ & 836 & $0.860 \pm 0.048$ & $0.972 \pm 0.018$ \\
\hline 805 & $0.922 \pm 0.028$ & $0.954 \pm 0.023$ & 837 & $0.986 \pm 0.042$ & $0.973 \pm 0.027$ \\
\hline 806 & $0.884 \pm 0.020$ & $0.958 \pm 0.028$ & 840 & $0.941 \pm 0.035$ & $0.973 \pm 0.023$ \\
\hline 810 & $0.911 \pm 0.099$ & $0.946 \pm 0.033$ & 841 & $0.938 \pm 0.026$ & $0.916 \pm 0.047$ \\
\hline 811 & $0.846 \pm 0.060$ & $0.973 \pm 0.032$ & 846 & $0.941 \pm 0.026$ & $0.861 \pm 0.057$ \\
\hline 813 & $0.961 \pm 0.066$ & $0.930 \pm 0.035$ & 848 & $0.909 \pm 0.033$ & $0.790 \pm 0.028$ \\
\hline 814 & $0.924 \pm 0.077$ & $0.989 \pm 0.018$ & 849 & $0.953 \pm 0.030$ & $0.840 \pm 0.055$ \\
\hline 817 & $0.986 \pm 0.048$ & - & 850 & $0.905 \pm 0.038$ & $0.919 \pm 0.035$ \\
\hline 818 & $0.945 \pm 0.079$ & $0.957 \pm 0.016$ & 851 & $0.883 \pm 0.028$ & $0.881 \pm 0.039$ \\
\hline 819 & $0.845 \pm 0.089$ & $0.961 \pm 0.030$ & 852 & $0.874 \pm 0.051$ & $0.928 \pm 0.036$ \\
\hline 820 & $0.918 \pm 0.092$ & $0.945 \pm 0.028$ & 853 & $0.920 \pm 0.044$ & $0.830 \pm 0.047$ \\
\hline 821 & $0.812 \pm 0.092$ & $0.955 \pm 0.034$ & 854 & $0.873 \pm 0.063$ & $0.887 \pm 0.044$ \\
\hline 822 & $0.889 \pm 0.072$ & $0.967 \pm 0.029$ & 855 & $0.843 \pm 0.061$ & $0.933 \pm 0.030$ \\
\hline 823 & $0.872 \pm 0.071$ & $0.934 \pm 0.042$ & 856 & $0.867 \pm 0.063$ & $0.965 \pm 0.020$ \\
\hline 825 & $0.893 \pm 0.045$ & $0.917 \pm 0.034$ & & & \\
\hline
\end{tabular}

$\left({ }^{*}\right)$ These $P R E_{a m p}$ were not used in the PPT analysis since their values were significantly affected by the fast timescale of the highly flexible loop. 
Table S4. Plane restraints used for the TMD-CT and MPER-TMD-CT structure calculation. $r_{Z}=0$ corresponds to the bicelle center. Plane restraints for the MPER and CT are shown in light blue and green, respectively; those for the TMD structure (internal calibration) are shown in red.

\begin{tabular}{|c|c|c|c|c|c|c|c|c|}
\hline Residue & $\begin{array}{c}\text { TMD-CT } \\
r_{z}(\AA)\end{array}$ & $\begin{array}{c}\text { MPER- } \\
\text { TMD-CT } \\
r_{Z}(\AA)\end{array}$ & Residue & $\begin{array}{c}\text { TMD-CT } \\
r_{z}(\AA)\end{array}$ & $\begin{array}{c}\text { MPER- } \\
\text { TMD-CT } \\
r_{Z}(\AA ̊)\end{array}$ & Residue & $\begin{array}{c}\text { TMD-CT } \\
r_{Z}(\AA)\end{array}$ & $\begin{array}{c}\text { MPER- } \\
\text { TMD-CT } \\
r_{Z}(\AA)\end{array}$ \\
\hline 678 & - & $17.8 \pm 1.0$ & 712 & $-22.1 \pm 1.0$ & $-22.1 \pm 1.0$ & 796 & $-16.6 \pm 1.0$ & $-16.6 \pm 1.0$ \\
\hline 679 & $24.1 \pm 1.0$ & $18.4 \pm 1.0$ & 713 & $-23.5 \pm 1.0$ & $-23.5 \pm 1.0$ & 799 & $-17.0 \pm 1.0$ & $-17.0 \pm 1.0$ \\
\hline 680 & $23.5 \pm 1.0$ & $17.5 \pm 1.0$ & 715 & $19.0 \pm 1.0$ & $19.0 \pm 1.0$ & 800 & $-18.6 \pm 1.0$ & $-18.6 \pm 1.0$ \\
\hline 681 & $21.5 \pm 1.0$ & $18.1 \pm 1.0$ & 716 & $-21.7 \pm 1.0$ & $-21.7 \pm 1.0$ & 801 & $-21.2 \pm 1.0$ & $-21.2 \pm 1.0$ \\
\hline 682 & $19.7 \pm 1.0$ & $17.8 \pm 1.0$ & 739 & $-19.1 \pm 1.0$ & $-19.1 \pm 1.0$ & 802 & $-16.9 \pm 1.0$ & $-16.9 \pm 1.0$ \\
\hline 683 & $19.1 \pm 1.0$ & $16.5 \pm 1.0$ & 742 & $-19.3 \pm 1.0$ & $-19.3 \pm 1.0$ & 803 & $-16.5 \pm 1.0$ & $-16.5 \pm 1.0$ \\
\hline 684 & $17.7 \pm 1.0$ & $16.7 \pm 1.0$ & 744 & $-18.5 \pm 1.0$ & $-18.5 \pm 1.0$ & 804 & $-17.8 \pm 1.0$ & $-17.8 \pm 1.0$ \\
\hline 685 & $15.5 \pm 1.0$ & $15.3 \pm 1.0$ & 747 & $-20.5 \pm 1.0$ & $-20.5 \pm 1.0$ & 805 & $-17.1 \pm 1.0$ & $-17.1 \pm 1.0$ \\
\hline 686 & $14.0 \pm 1.0$ & $13.5 \pm 1.0$ & 748 & $-17.7 \pm 1.0$ & $-17.7 \pm 1.0$ & 806 & $-17.4 \pm 1.0$ & $-17.4 \pm 1.0$ \\
\hline 687 & $13.0 \pm 1.0$ & $12.6 \pm 1.0$ & 750 & $-18.6 \pm 1.0$ & $-18.6 \pm 1.0$ & 810 & $-17.8 \pm 1.0$ & $-17.8 \pm 1.0$ \\
\hline 688 & $11.2 \pm 1.0$ & - & 753 & $-16.3 \pm 1.0$ & $-16.3 \pm 1.0$ & 811 & $-16.2 \pm 1.0$ & $-16.2 \pm 1.0$ \\
\hline 689 & $9.3 \pm 1.0$ & - & 754 & $-19.3 \pm 1.0$ & $-19.3 \pm 1.0$ & 813 & $-20.2 \pm 1.0$ & $-20.2 \pm 1.0$ \\
\hline 690 & $8.5 \pm 1.0$ & - & 755 & $-17.6 \pm 1.0$ & $-17.6 \pm 1.0$ & 818 & $-16.9 \pm 1.0$ & $-16.9 \pm 1.0$ \\
\hline 691 & $6.7 \pm 1.0$ & - & 756 & $-17.5 \pm 1.0$ & $-17.5 \pm 1.0$ & 819 & $-16.3 \pm 1.0$ & $-16.3 \pm 1.0$ \\
\hline 692 & $4.3 \pm 1.0$ & - & 758 & $-17.9 \pm 1.0$ & $-17.9 \pm 1.0$ & 820 & $-17.9 \pm 1.0$ & $-17.9 \pm 1.0$ \\
\hline 693 & $3.0 \pm 1.0$ & - & 762 & $-18.8 \pm 1.0$ & $-18.8 \pm 1.0$ & 821 & $-16.0 \pm 1.0$ & $-16.0 \pm 1.0$ \\
\hline 694 & $1.8 \pm 1.0$ & - & 763 & $-16.5 \pm 1.0$ & $-16.5 \pm 1.0$ & 822 & $-17.1 \pm 1.0$ & $-17.1 \pm 1.0$ \\
\hline 695 & $-0.4 \pm 1.0$ & $-0.4 \pm 1.0$ & 770 & $-16.0 \pm 1.0$ & $-16.0 \pm 1.0$ & 823 & $-18.0 \pm 1.0$ & $-18.0 \pm 1.0$ \\
\hline 696 & $-2.4 \pm 1.0$ & $-2.4 \pm 1.0$ & 771 & $-18.6 \pm 1.0$ & $-18.6 \pm 1.0$ & 825 & $-19.2 \pm 1.0$ & $-19.2 \pm 1.0$ \\
\hline 697 & $-4.3 \pm 1.0$ & $-4.3 \pm 1.0$ & 772 & $-18.2 \pm 1.0$ & $-18.2 \pm 1.0$ & 829 & $-18.1 \pm 1.0$ & $-18.1 \pm 1.0$ \\
\hline 698 & $-6.6 \pm 1.0$ & $-6.6 \pm 1.0$ & 774 & $-17.8 \pm 1.0$ & $-17.8 \pm 1.0$ & 832 & $-19.2 \pm 1.0$ & $-19.2 \pm 1.0$ \\
\hline 699 & $-8.4 \pm 1.0$ & $-8.4 \pm 1.0$ & 776 & $-18.2 \pm 1.0$ & $-18.2 \pm 1.0$ & 833 & $-16.9 \pm 1.0$ & $-16.9 \pm 1.0$ \\
\hline 700 & $-10.0 \pm 1.0$ & $-10.0 \pm 1.0$ & 779 & $-15.8 \pm 1.0$ & $-15.8 \pm 1.0$ & 834 & $-19.8 \pm 1.0$ & $-19.8 \pm 1.0$ \\
\hline 701 & $-11.7 \pm 1.0$ & $-11.7 \pm 1.0$ & 782 & $-16.9 \pm 1.0$ & $-16.9 \pm 1.0$ & 835 & $-21.4 \pm 1.0$ & $-21.4 \pm 1.0$ \\
\hline 702 & $-13.1 \pm 1.0$ & $-13.1 \pm 1.0$ & 785 & $-16.6 \pm 1.0$ & $-16.6 \pm 1.0$ & 836 & $-16.9 \pm 1.0$ & $-16.9 \pm 1.0$ \\
\hline 703 & $-15.2 \pm 1.0$ & $-15.2 \pm 1.0$ & 786 & $-16.4 \pm 1.0$ & $-16.4 \pm 1.0$ & 841 & $-19.9 \pm 1.0$ & $-19.9 \pm 1.0$ \\
\hline 704 & $-17.6 \pm 1.0$ & $-17.6 \pm 1.0$ & 788 & $-16.7 \pm 1.0$ & $-16.7 \pm 1.0$ & 850 & $-19.6 \pm 1.0$ & $-19.6 \pm 1.0$ \\
\hline 705 & $-18.2 \pm 1.0$ & $-18.2 \pm 1.0$ & 789 & $-20.8 \pm 1.0$ & $-20.8 \pm 1.0$ & 851 & $-20.3 \pm 1.0$ & $-20.3 \pm 1.0$ \\
\hline 706 & $-18.9 \pm 1.0$ & $-18.9 \pm 1.0$ & 790 & $-20.1 \pm 1.0$ & $-20.1 \pm 1.0$ & 852 & $-18.3 \pm 1.0$ & $-18.3 \pm 1.0$ \\
\hline 707 & $-21.3 \pm 1.0$ & $-21.3 \pm 1.0$ & 791 & $-16.6 \pm 1.0$ & $-16.6 \pm 1.0$ & 854 & $-19.7 \pm 1.0$ & $-19.7 \pm 1.0$ \\
\hline 708 & $-23.2 \pm 1.0$ & $-23.2 \pm 1.0$ & 792 & $-18.3 \pm 1.0$ & $-18.3 \pm 1.0$ & 855 & $-17.4 \pm 1.0$ & $-17.4 \pm 1.0$ \\
\hline 709 & $-22.8 \pm 1.0$ & $-22.8 \pm 1.0$ & 794 & $-18.8 \pm 1.0$ & $-18.8 \pm 1.0$ & 856 & $-16.6 \pm 1.0$ & $-16.6 \pm 1.0$ \\
\hline 710 & $-23.8 \pm 1.0$ & $-23.8 \pm 1.0$ & 795 & $-17.8 \pm 1.0$ & $-17.8 \pm 1.0$ & & & \\
\hline
\end{tabular}


Table S5. TMD-CT NMR structure calculation and refinement statistics.

\begin{tabular}{|c|c|c|c|}
\hline NMR distance, dihedral and plane restraints ${ }^{a}$ & $\begin{array}{c}\text { TMD } \\
(677-710)\end{array}$ & $\begin{array}{c}C^{C^{\text {LLP2 }}} \\
(711-785)\end{array}$ & $\begin{array}{c}\text { CT }^{\text {LLP3-LLP1 }} \\
(786-856)\end{array}$ \\
\hline Distance restraints from $\mathrm{NOE}^{\mathrm{b}}$ & 510 & 549 & 156 \\
\hline Short-range intramolecular $(|i-j| \leq 4)$ & 459 & 465 & 69 \\
\hline Long-range intramolecular $(|i-j| \geq 5)$ & 0 & 24 & 45 \\
\hline Intermolecular & 51 & 60 & 42 \\
\hline Total plane restraints $^{c}$ & 96 & \multicolumn{2}{|c|}{195} \\
\hline Total dihedral angle restraints ${ }^{d}$ & & \multicolumn{2}{|c|}{852} \\
\hline$\Phi($ TALOS) & & \multicolumn{2}{|c|}{426} \\
\hline$\psi($ TALOS) & & \multicolumn{2}{|c|}{426} \\
\hline \multicolumn{4}{|l|}{ Structure statistics ${ }^{\text {e }}$} \\
\hline \multicolumn{4}{|l|}{ Violations (mean \pm s.d.) } \\
\hline Distance restraints $(\AA ̊)$ & & \multicolumn{2}{|c|}{$0.182 \pm 0.005$} \\
\hline Plane restraints $(\AA)$ & & \multicolumn{2}{|c|}{$0.095 \pm 0.041$} \\
\hline Dihedral angle restraints $\left({ }^{\circ}\right)$ & & \multicolumn{2}{|c|}{$1.066 \pm 0.041$} \\
\hline \multicolumn{4}{|l|}{ Deviations from idealized geometry } \\
\hline Bond lengths ( $(̊)$ & & \multicolumn{2}{|c|}{$0.009 \pm 0.000$} \\
\hline Bond angles $\left({ }^{\circ}\right)$ & & \multicolumn{2}{|c|}{$1.030 \pm 0.011$} \\
\hline Impropers $\left({ }^{\circ}\right)$ & & \multicolumn{2}{|c|}{$0.827 \pm 0.018$} \\
\hline \multicolumn{4}{|l|}{ Average pairwise r.m.s. deviation $(\AA)^{f}$} \\
\hline Heavy & & \multicolumn{2}{|c|}{1.804} \\
\hline Backbone & & \multicolumn{2}{|c|}{1.443} \\
\hline
\end{tabular}

a The numbers of restraints are summed over all three subunits.

${ }^{b}$ For residues 786-856, all NOE restraints were obtained in the current study. For residues 679-710 and 711-785, the NOE restraints were taken from our earlier NMR studies of the TMD and TMD-CT ${ }^{\text {LP2 }}$ ( refs $^{1,5}$ ).

${ }^{c}$ For residues 711-856, plane restraints were derived using $P R E_{a m p}$ values obtained in the current study. For residues 679-710, plane restraints were directly taken from our earlier NMR study of the TMD (internal calibration) ${ }^{1}$.

"Backbone $\phi$ and $\psi$ restraints and their respective uncertainties were obtained from the "GOOD" dihedrals generated by TALOS+ based on the backbone chemical shift values.

e Statistics are calculated and averaged over an ensemble of the 15 lowest energy structures out of 150 calculated structures.

${ }^{\mathrm{f}}$ The precision of the atomic coordinates is defined as the average r.m.s. difference between the 15 final structures and their mean coordinates (flexible residues $711-740$ have been omitted from the comparison). 
Table S6. MPER-TMD-CT NMR model calculation and refinement statistics.

\begin{tabular}{|c|c|c|c|c|}
\hline NMR distance, dihedral and plane restraints ${ }^{a}$ & $\begin{array}{c}\text { MPER-TMD } \\
(660-693)\end{array}$ & $\begin{array}{c}\text { TMD } \\
(694-710)\end{array}$ & $\begin{array}{c}C^{\text {LLP2 }} \\
(711-785)\end{array}$ & $\begin{array}{l}\text { CT }^{\text {LLP3-LLP1 }} \\
(786-856)\end{array}$ \\
\hline Distance restraints from $\mathrm{NOE}^{\mathrm{b}}$ & 333 & 429 & 549 & 156 \\
\hline Short-range intramolecular $(|i-j| \leq 4)$ & 303 & 387 & 465 & 69 \\
\hline Long-range intramolecular $(|i-j| \geq 5)$ & 6 & 0 & 24 & 45 \\
\hline Intermolecular & 24 & 42 & 60 & 42 \\
\hline Total plane restraints ${ }^{c}$ & 30 & 48 & \multicolumn{2}{|c|}{195} \\
\hline Total dihedral angle restraints ${ }^{d}$ & & \multicolumn{3}{|c|}{912} \\
\hline$\Phi($ TALOS) & & \multicolumn{3}{|c|}{456} \\
\hline$\psi($ TALOS) & & \multicolumn{3}{|c|}{456} \\
\hline \multicolumn{5}{|l|}{ Structure statistics ${ }^{\text {e }}$} \\
\hline \multicolumn{5}{|l|}{ Violations (mean \pm s.d.) } \\
\hline Distance restraints $(\AA ̊)$ & & \multicolumn{3}{|c|}{$0.152 \pm 0.004$} \\
\hline Plane restraints $(\AA)$ & & \multicolumn{3}{|c|}{$0.115 \pm 0.037$} \\
\hline Dihedral angle restraints $\left({ }^{\circ}\right)$ & & \multicolumn{3}{|c|}{$0.971 \pm 0.067$} \\
\hline \multicolumn{5}{|l|}{ Deviations from idealized geometry } \\
\hline Bond lengths $(\AA ̊)$ & & \multicolumn{3}{|c|}{$0.009 \pm 0.000$} \\
\hline Bond angles $\left({ }^{\circ}\right)$ & & \multicolumn{3}{|c|}{$1.013 \pm 0.013$} \\
\hline Impropers $\left({ }^{\circ}\right)$ & & \multicolumn{3}{|c|}{$0.781 \pm 0.019$} \\
\hline \multicolumn{5}{|l|}{ Average pairwise r.m.s. deviation $(\AA)^{f}$} \\
\hline Heavy & & \multicolumn{3}{|c|}{1.711} \\
\hline Backbone & & \multicolumn{3}{|c|}{1.120} \\
\hline
\end{tabular}

a The numbers of restraints are summed over all three subunits.

${ }^{b}$ For residues 786-856, all NOE restraints were obtained in the current study. For the MPER and first half of the TMD (residues 660-693), the NOE restraints were taken from our earlier NMR study of the MPER-TMD ${ }^{6}$; for the second half of the TMD (residues 694-710) and for the CT ${ }^{\text {LLP2 }}$ (residues 711-785), the NOE restraints were taken from our earlier NMR study of the TMD and TMD-CT ${ }^{\text {LLP2 }}$ (refs ${ }^{1,5}$ ).

${ }^{c}$ For the residues 711-856, plane restraints were derived using the $P R E_{a m p}$ values obtained in the current study. For the MPER and first half of the TMD (residues 660-693), plane restraints were derived using the $P R E_{a m p}$ values taken from our earlier NMR study of the MPER-TMD ${ }^{6}$; for the second half of the TMD (residues 694-710), plane restraints were directly taken from our earlier NMR study of the TMD (internal calibration) ${ }^{1}$.

d Backbone $\phi$ and $\psi$ restraints and their respective uncertainties were obtained from the "GOOD" dihedrals generated by TALOS+ based on the backbone chemical shift values. For residues 694-856, all dihedral angle restraints were obtained in the current study; for residues 660-693, all dihedral angle restraints were taken from our earlier NMR study of the MPER-TMD ${ }^{6}$.

e Statistics are calculated and averaged over an ensemble of the 15 lowest energy structures out of 150 calculated structures.

${ }^{f}$ The precision of the atomic coordinates is defined as the average r.m.s. difference between the 15 final structures and their mean coordinates (flexible residues 711-740 have been omitted from the comparison). 
a)

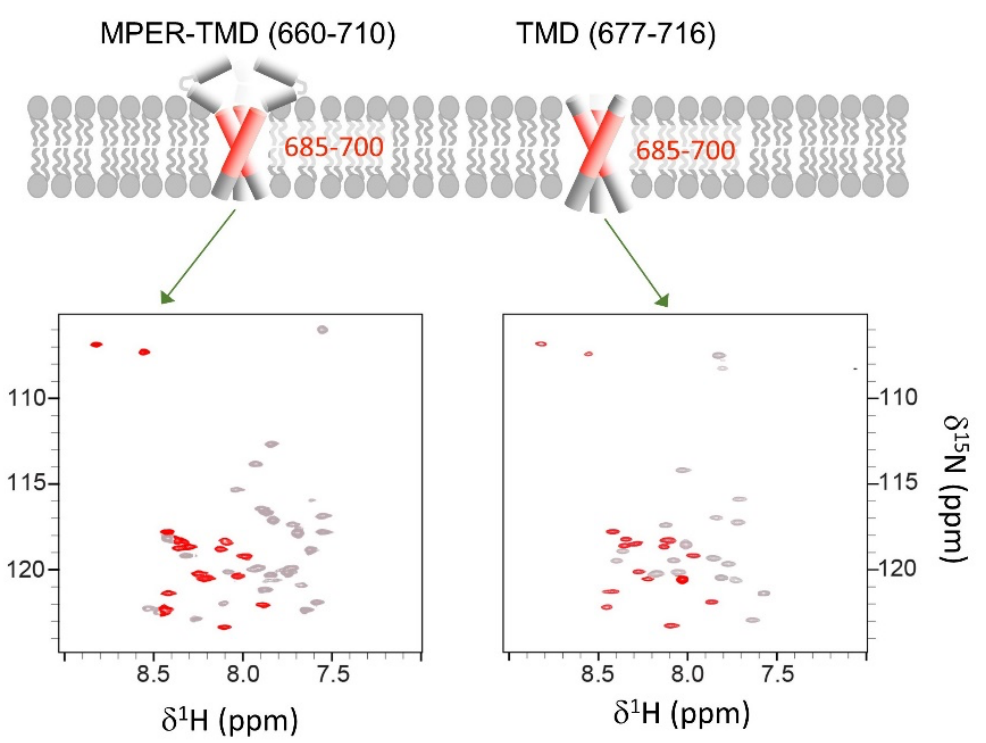

b)

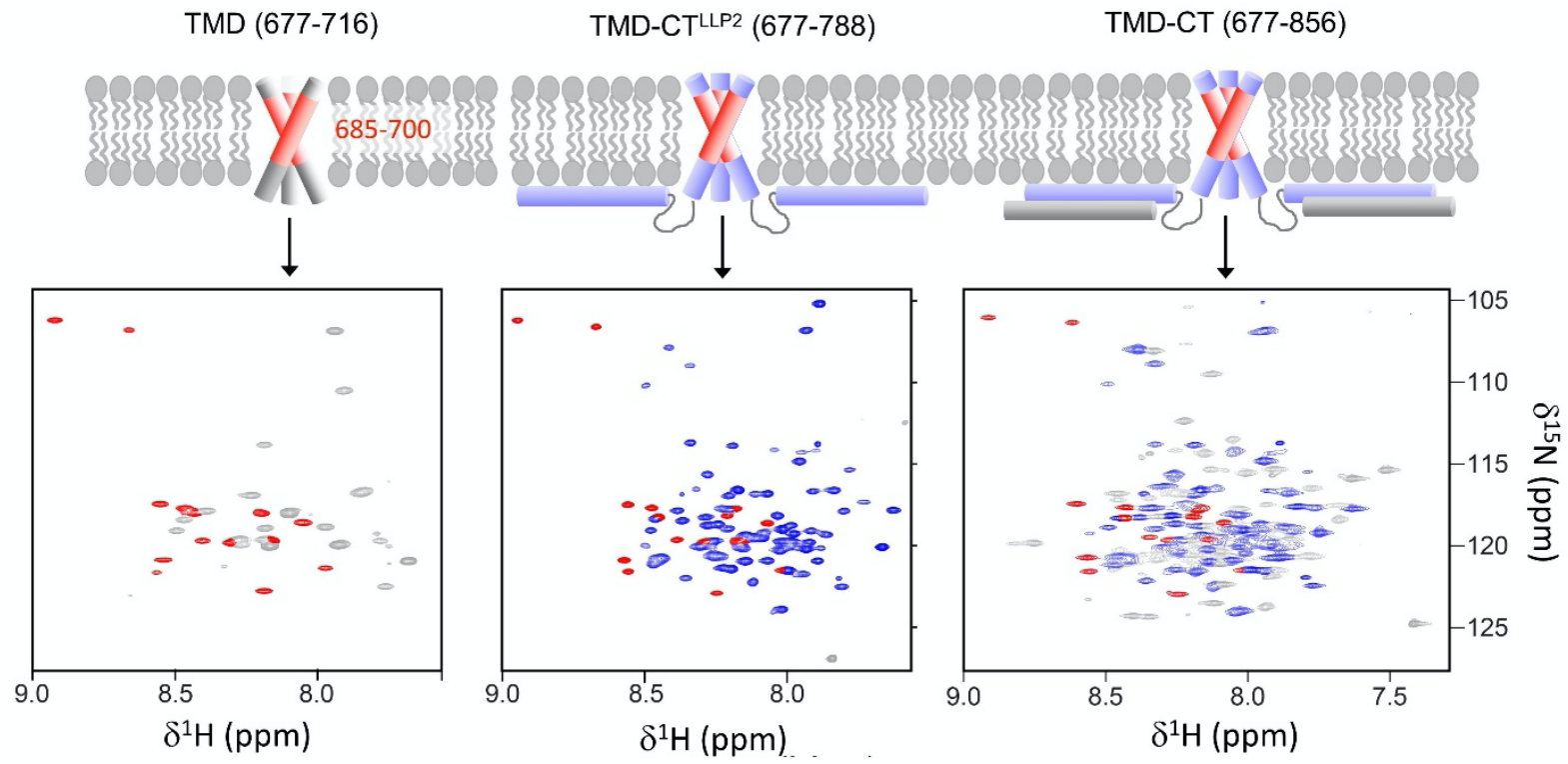

Figure S1. NMR spectra of the membrane components of HIV-1 Env in bicelles. (a) Comparison of the $2 \mathrm{D}{ }^{1} \mathrm{H}-{ }^{15} \mathrm{~N}$ TROSY-HSQC spectra of the MPER-TMD and TMD fragments defined in Fig. 1. The peaks from the TMD core (residues 685-700) are shown in red. (b) Comparison of the $2 \mathrm{D}{ }^{1} \mathrm{H}-{ }^{15} \mathrm{~N}$ TROSY-HSQC spectra of the TMD, TMD-CTLP2 and TMDCT fragments. The peaks from the TMD core and the $C T^{L L P 2}$ region are shown in red and blue, respectively. Note: the bicelles in (a) contains a small amount of $\mathrm{DH}_{7} \mathrm{PC}(\sim 10 \%$ of the short-chain detergent) needed for sample reconstitution $^{6}$, and this makes the bicelle conditions in (a) and (b) slightly different. 


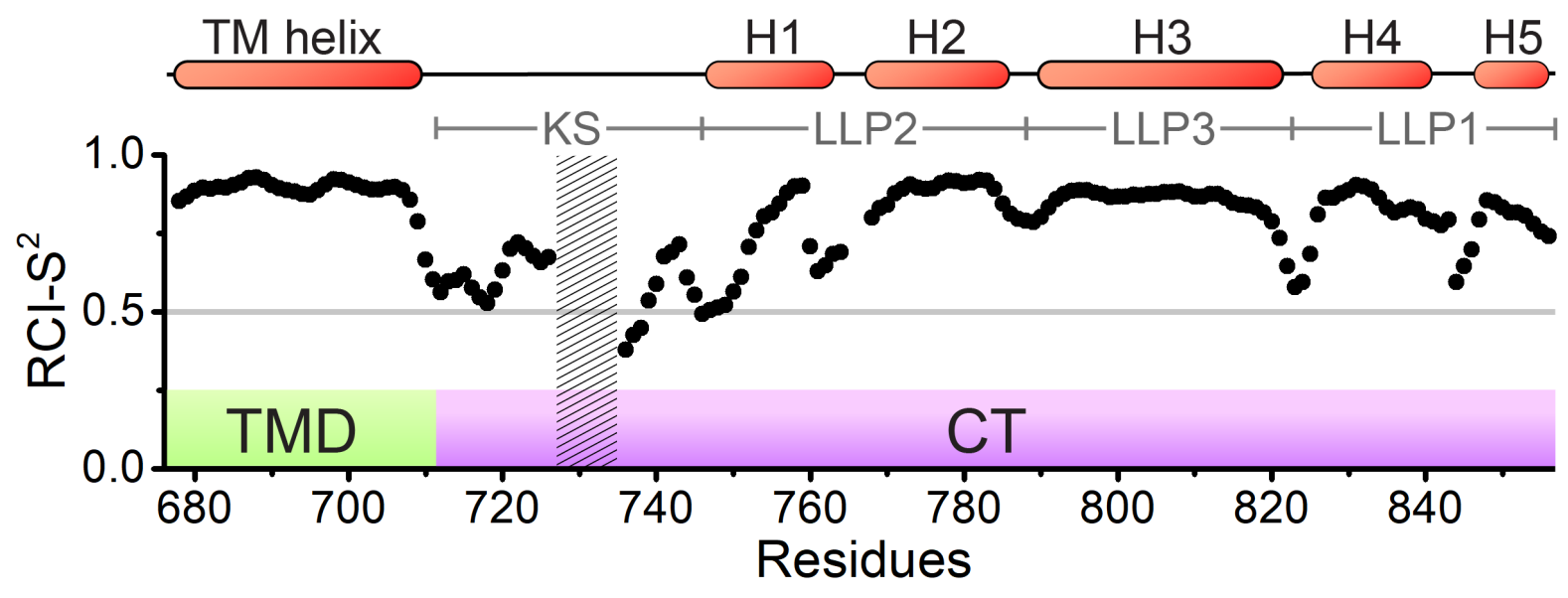

Figure S2. Secondary structure analysis of the TMD-CT. The random coil index (RCI)-based order parameter $\left(\mathrm{S}^{2}\right)$ calculated from experimental chemical shifts $\left({ }^{15} \mathrm{~N},{ }^{13} \mathrm{C}^{\alpha}\right.$ and $\left.{ }^{13} \mathrm{C}^{\prime}\right)$ using TALOS$+{ }^{12}$ is shown for assigned residues. High and low values indicate structured and unstructured regions, respectively. The striped box corresponds to residues 726-736 (deleted in the construct). The $\alpha$-helical segments along the protein sequence are indicated by red bars. 


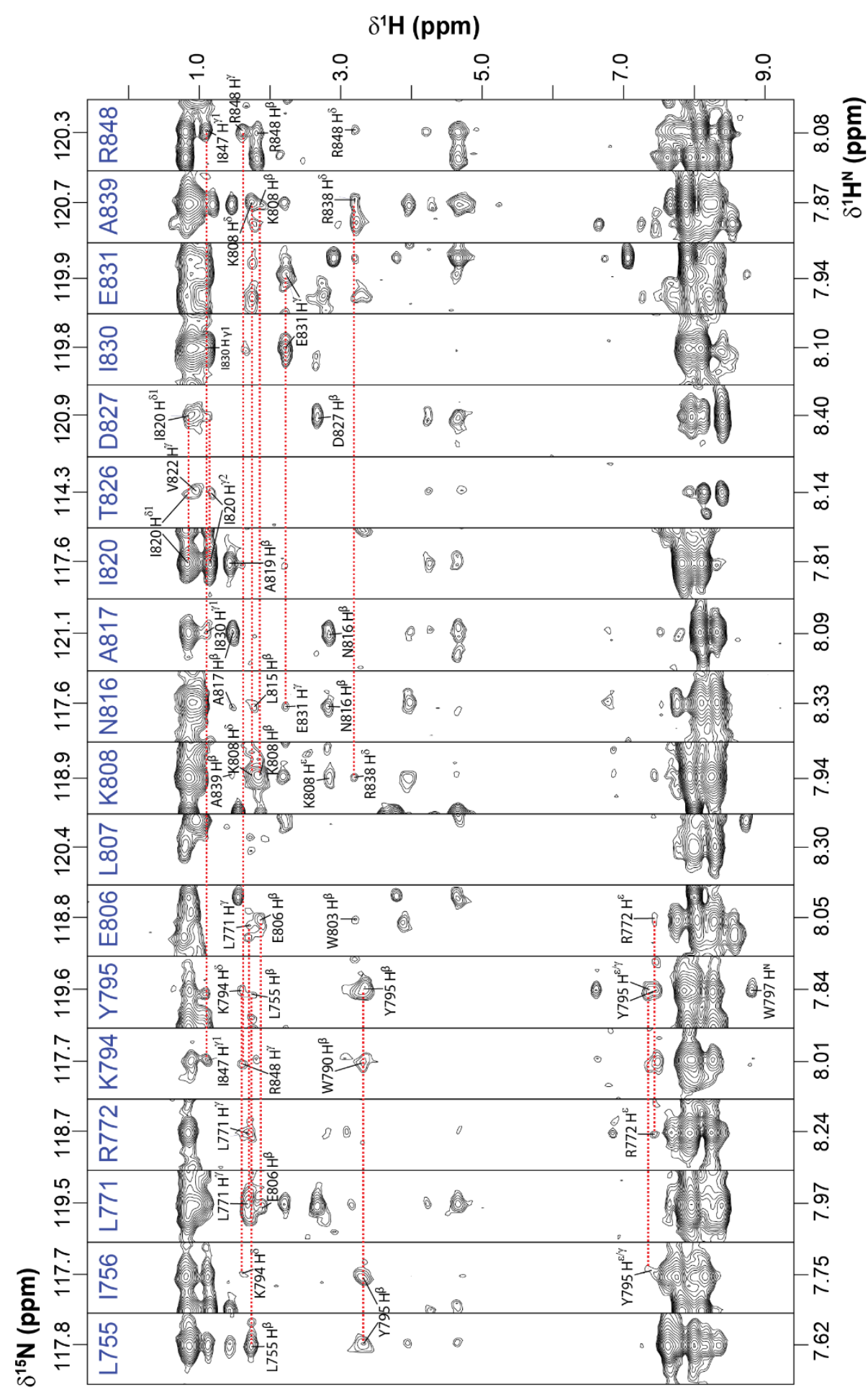

Figure S3. Examples of long-range NOEs used for determining the conformation of the CT ${ }^{\text {LP3-LLP1 }}$ region relative to the $C T^{L L P 2}$ region. Stripes shown are from the $3 \mathrm{D}^{15} \mathrm{~N}$-edited NOESY-TROSY-HSQC recorded at $21.1 \mathrm{~T}$ and $308 \mathrm{~K}$ with a NOE mixing time of $150 \mathrm{~ms}$. The red dashed lines indicate matching chemical shift between intra-residue and longrange NOEs. The spectrum was acquired using a $\sim 1.0 \mathrm{mM}\left({ }^{15} \mathrm{~N}, 85 \%{ }^{2} \mathrm{H}\right)$ - and (ILV) ${ }^{13} \mathrm{C}$-labeled TMD-CT sample reconstituted in bicelles with deuterated DMPC and DHPC acyl chains. 
a)

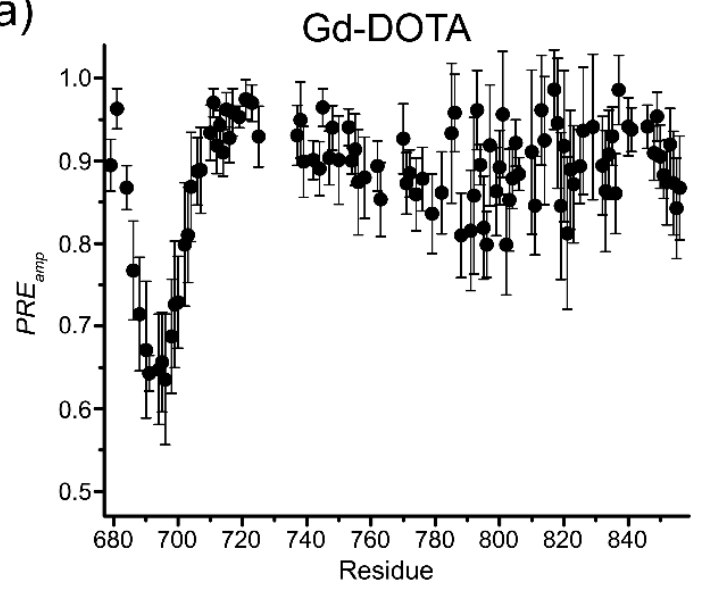

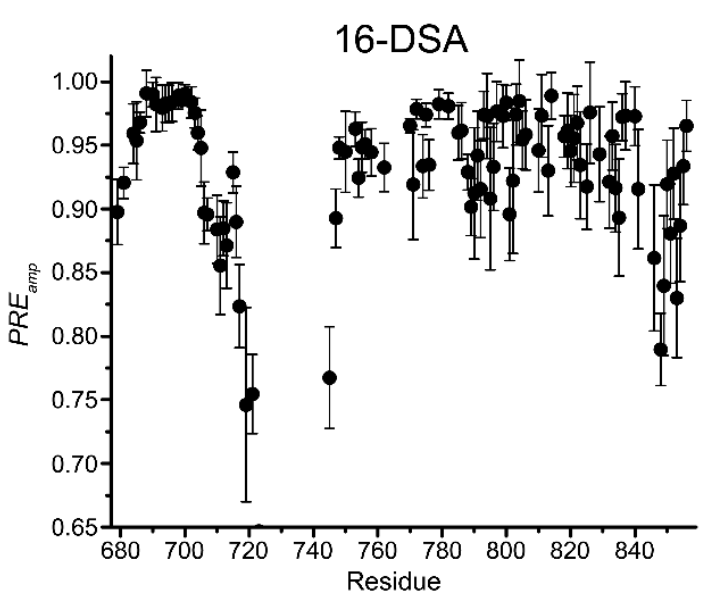

b)

TMD calibration (residues 679-710)
Plane restraints (residues 711-856)
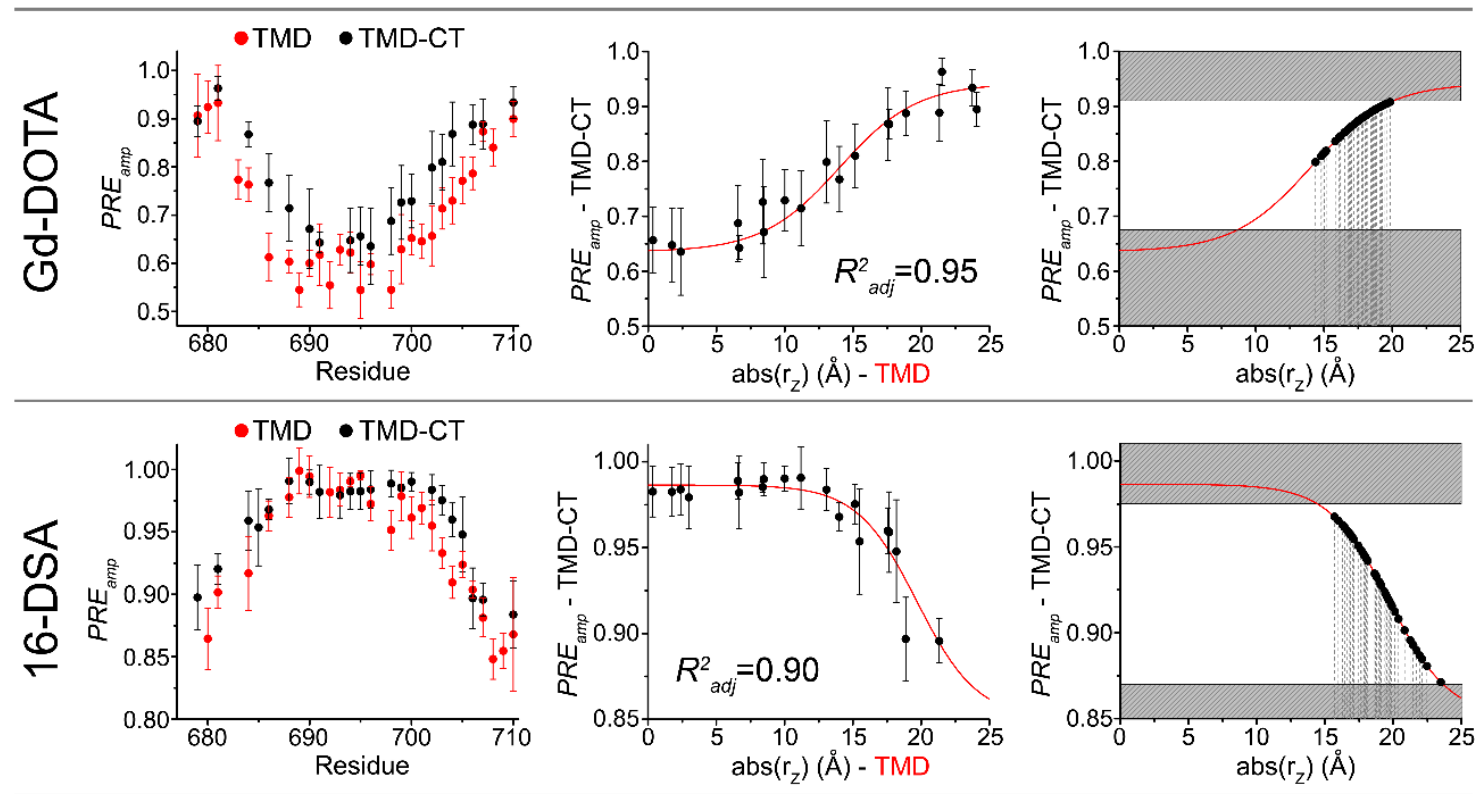

Figure S4. Derivation of the plane restraints used in the TMD-CT and MPER-TMD-CT structure calculation. (a) PREamp vs (residue number) plots for TMD-CT obtained from Gd-DOTA (left) and 16-DSA (right) titrations. Error bars represent the uncertainty derived from fitting error. Missing values are due to prolines, residues with overlapping cross-peaks or the deleted KS residues 726-736. (b) Assignment of plane restraints for residues 711-856 of the TMDCT using data from Gd-DOTA and 16-DSA titrations (top and bottom row, respectively). First, PRE amp vs (residue number) plots of residues 679-710 of the TMD-CT (black) and the TMD (red) were compared (left panel). Collectively, the two data sets indicate that residues 679-710 have the same membrane partition in both constructs. Second, the $P R E_{a m p}$ vs. (residue number) plot of the TMD-CT was converted to a $P R E_{a m p}$ vs. $r_{z}$ plot using the known TMD trimer structure (PDB ID: 5JYN). The PRE $E_{a m p}$ vs. $r_{z}$ profiles were then fitted to the sigmoidal function (Eq. S2) (middle panel). Third, the best-fit curves (red) were used to assign $r_{z}$ for residues 711-856 (right panel). Only $r_{z}$ in the PRE-sensitive regions of the curves were retained (white area). Finally, the calculated $r_{z}$ from Gd-DOTA and 16-DSA curves were averaged, merged into one single data set and used as plane restraints for the TMD-CT and MPER-TMD-CT structure calculation. 
a)
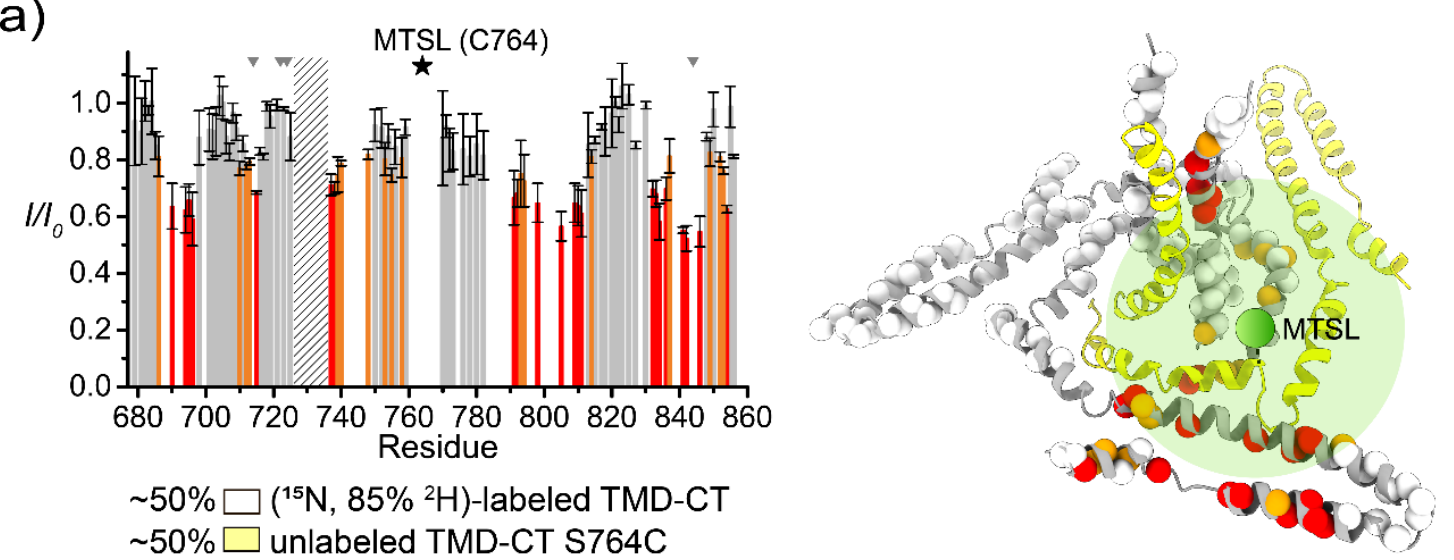

b)

$\sim 50 \% \square\left({ }^{15} \mathrm{~N}, 85 \%{ }^{2} \mathrm{H}\right)$-labeled TMD-CT

$\sim 50 \% \square$ unlabeled TMD-CT S764C

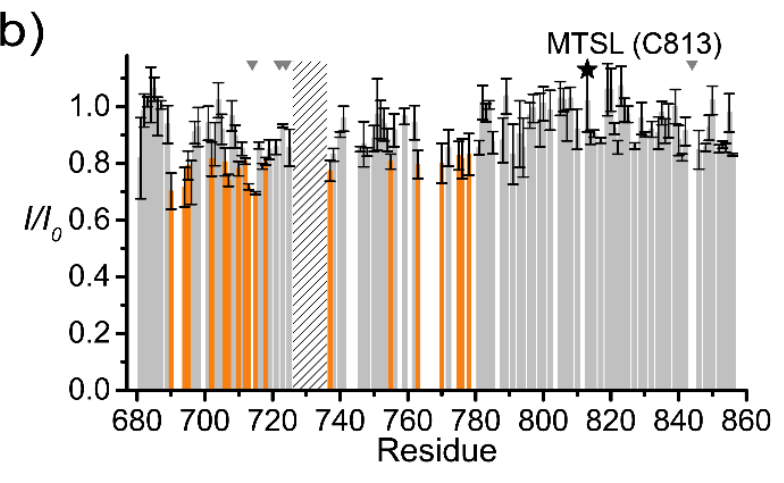

$\sim 50 \% \square\left({ }^{15} \mathrm{~N}, 85 \%{ }^{2} \mathrm{H}\right)$-labeled TMD-CT

$\sim 50 \% \square$ unlabeled TMD-CT S813C

c)
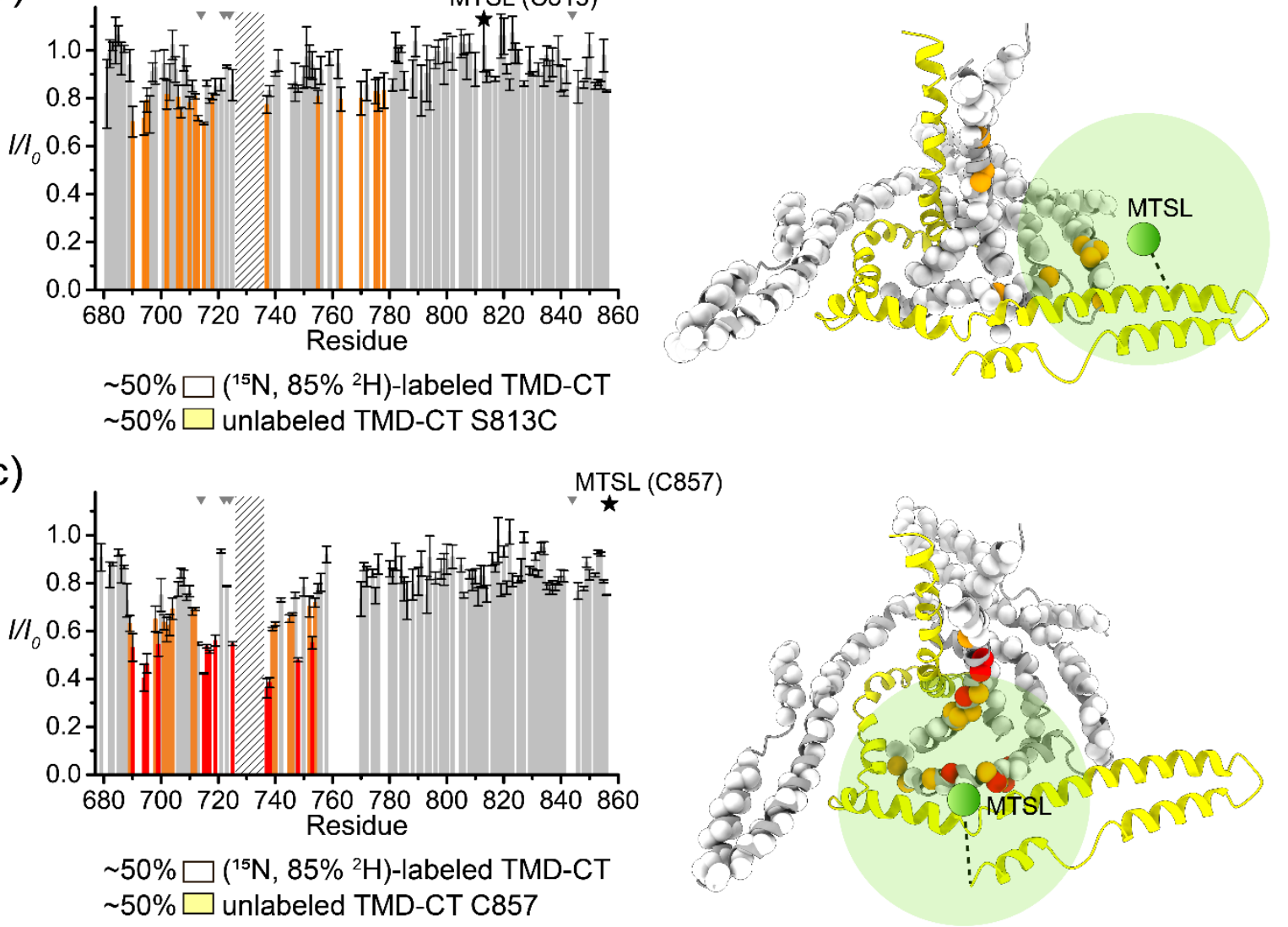

Figure S5. Validation of the TMD-CT structure by inter-chain PRE analysis. (a) Left: Residue-specific PRE $\left(I / I_{0}\right)$ plot of $\left({ }^{15} \mathrm{~N}, 85 \%{ }^{2} \mathrm{H}\right)$-labeled TMD-CT mixed with the TMD-CT S764C mutant MTSL-labeled at C764 at $\sim 1: 1$ molar ratio. Error bars represent the uncertainty derived from cross-peaks signal to noise. Missing bars are due to prolines or residues with overlapping peaks. The striped box corresponds to residues 726-736 (deleted in the construct), the grey triangles mark the proline residues and the asterisk indicates the position of the MTSL label. The three main PRE regimes are defined as strong (red), medium (orange) and weak (gray). On the right, the mapping of the PRE regimes onto the protein structure is shown. The unstructured KS (residues $711-736)$ is omitted for clarity. The $\left({ }^{15} \mathrm{~N}, 85 \%{ }^{2} \mathrm{H}\right)$ labeled TMD-CT and the MTSL-labeled S764C mutant are represented as gray and yellow ribbons, respectively. Amide protons for which accurate PRE measurement was feasible are shown as spheres and colored according to the PRE regimes defined above (red, orange and white). The green sphere represents the area affected by the paramagnetic tag. (b) Same as (a) but for MTSL labeled at C813. (c) Same as (a) but for MTSL labeled at C857. 
a)
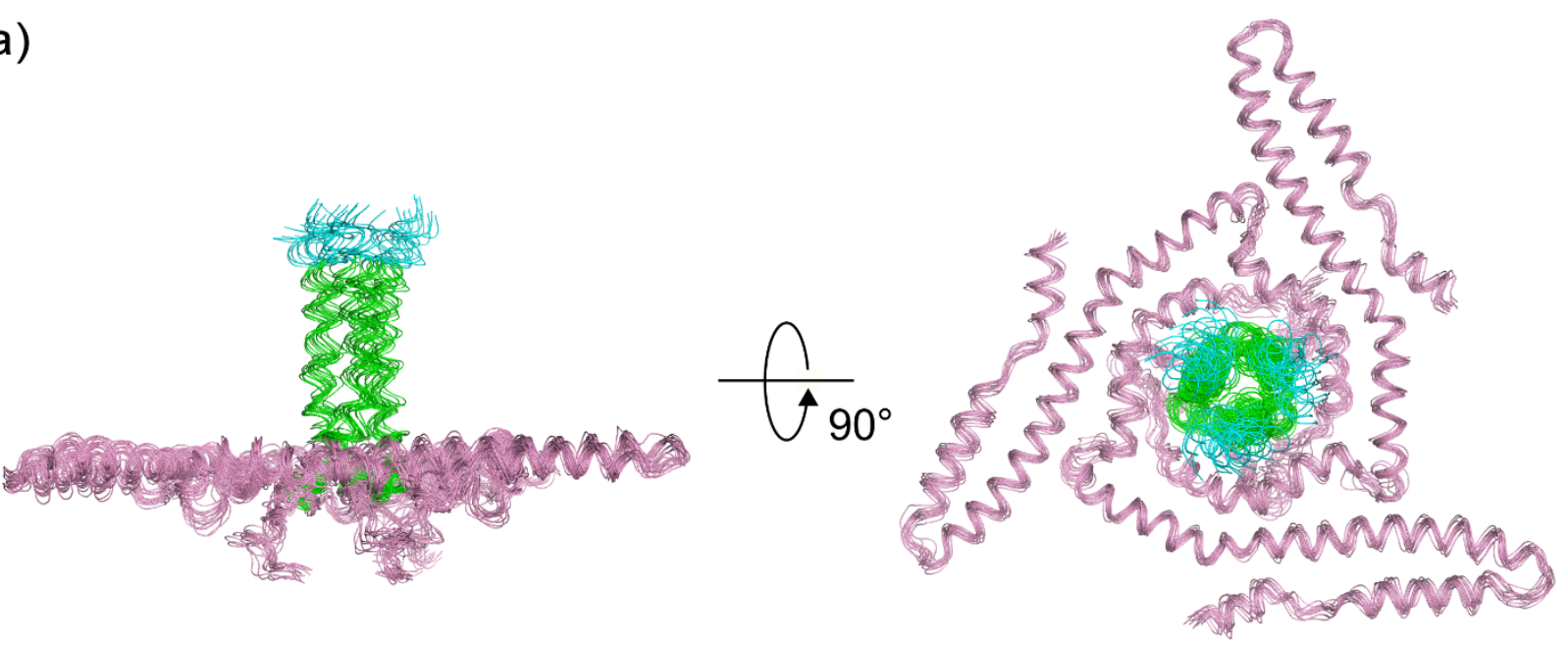

b)
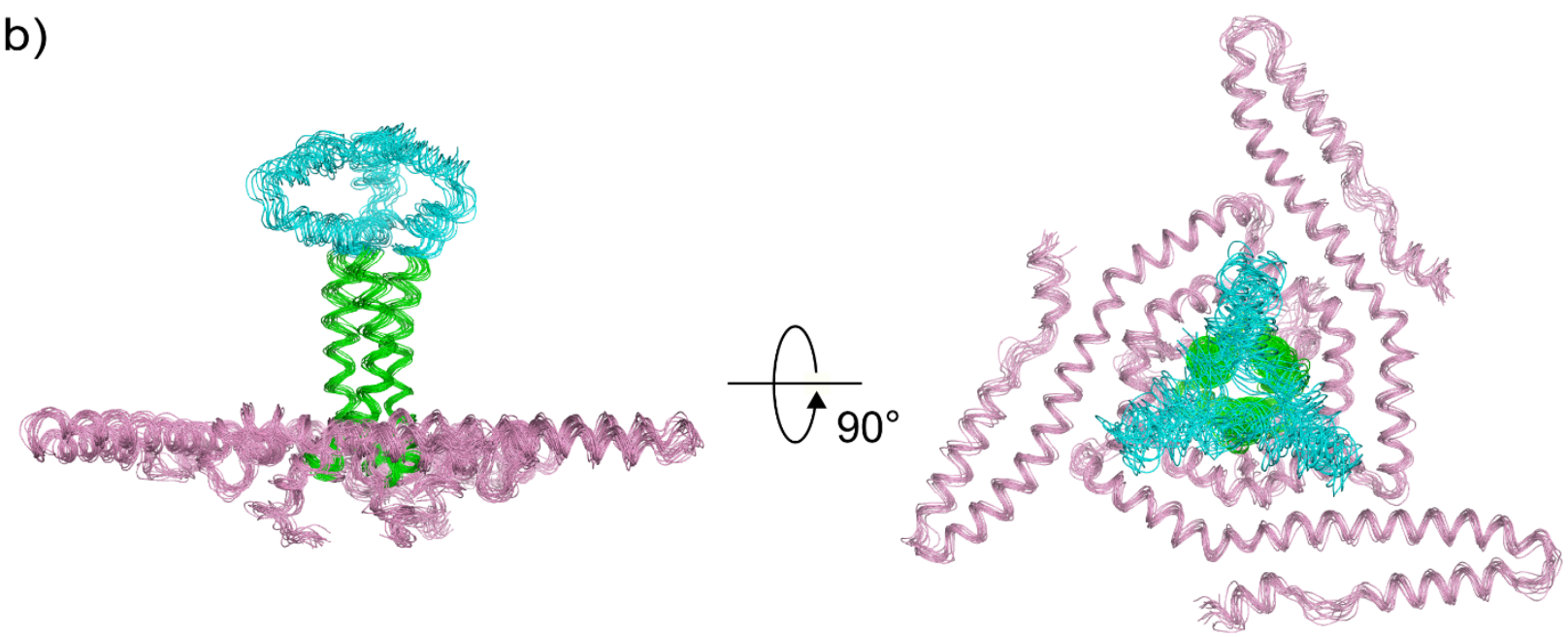

Figure S6. TMD-CT and MPER-TMD-CT structural ensembles. The MPER, TMD and CT are colored in cyan, green and pink, respectively. Structures are represented as thin ribbons and residues 726-736 have been omitted. (a) Side and top view of the NMR ensemble of the 15 lowest-energy TMD-CT structures calculated using NMR-derived structural restraints (Table S5). (b) Side and top view of the NMR ensemble of the 15 lowest-energy MPER-TMD-CT structures calculated using NMR-derived structural restraints (Table S6). 


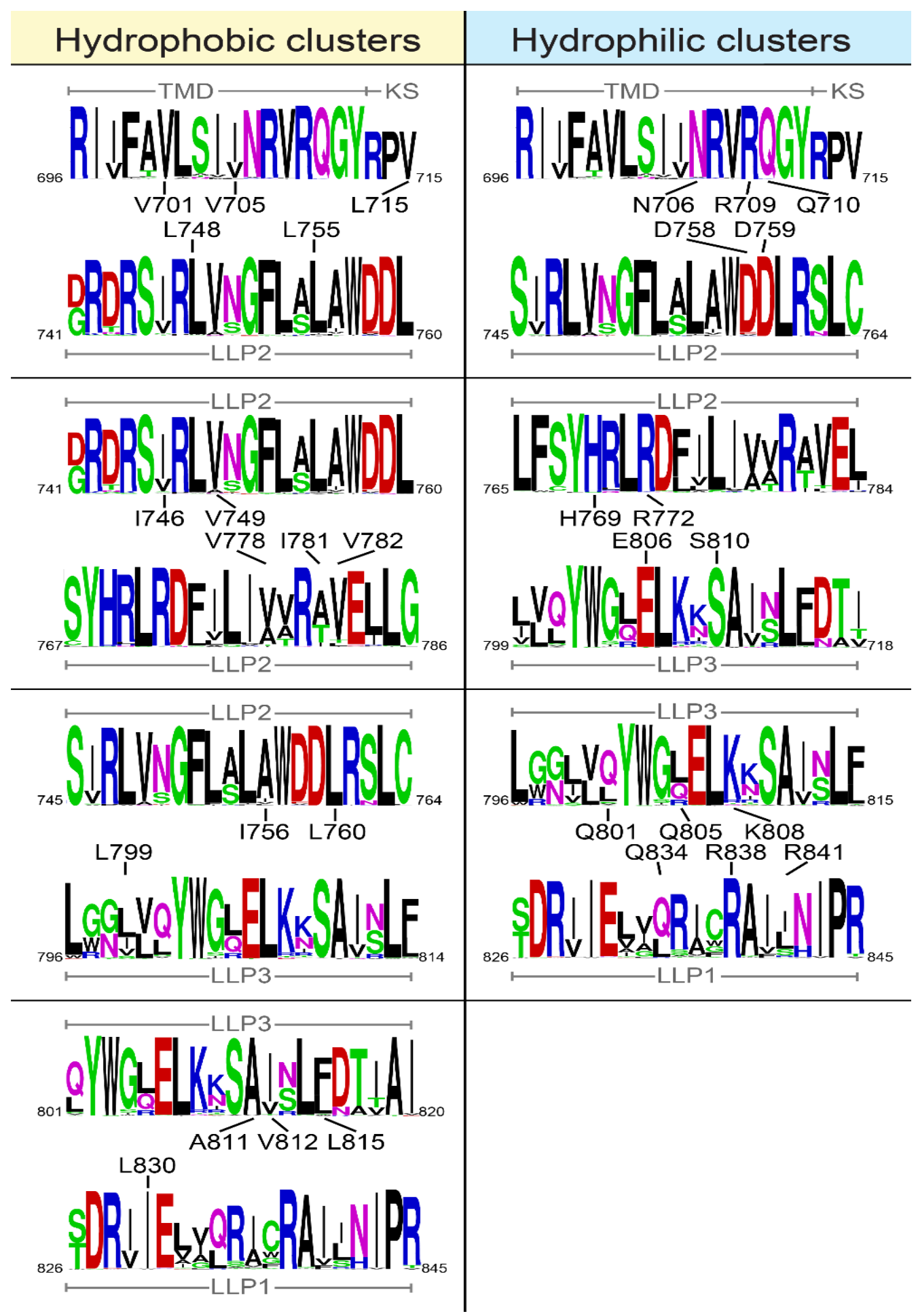

Figure S7. Conservation of residues forming the hydrophobic and hydrophilic clusters of the CT baseplate (Fig. 2c) across HIV-1 isolates. A total of 2,154 HIV-1 Env sequences from the $\mathrm{NBCl}$ database (https://blast.ncbi.nlm.nih.gov/Blast.cgi) were aligned and the resulting sequence logo was generated using WEBLOGO v2.8. $2^{28}$. Residues in the clusters are labeled. The result indicates that the polar/apolar nature of these clusters is highly conserved throughout HIV-1 variants. 
Rep 1
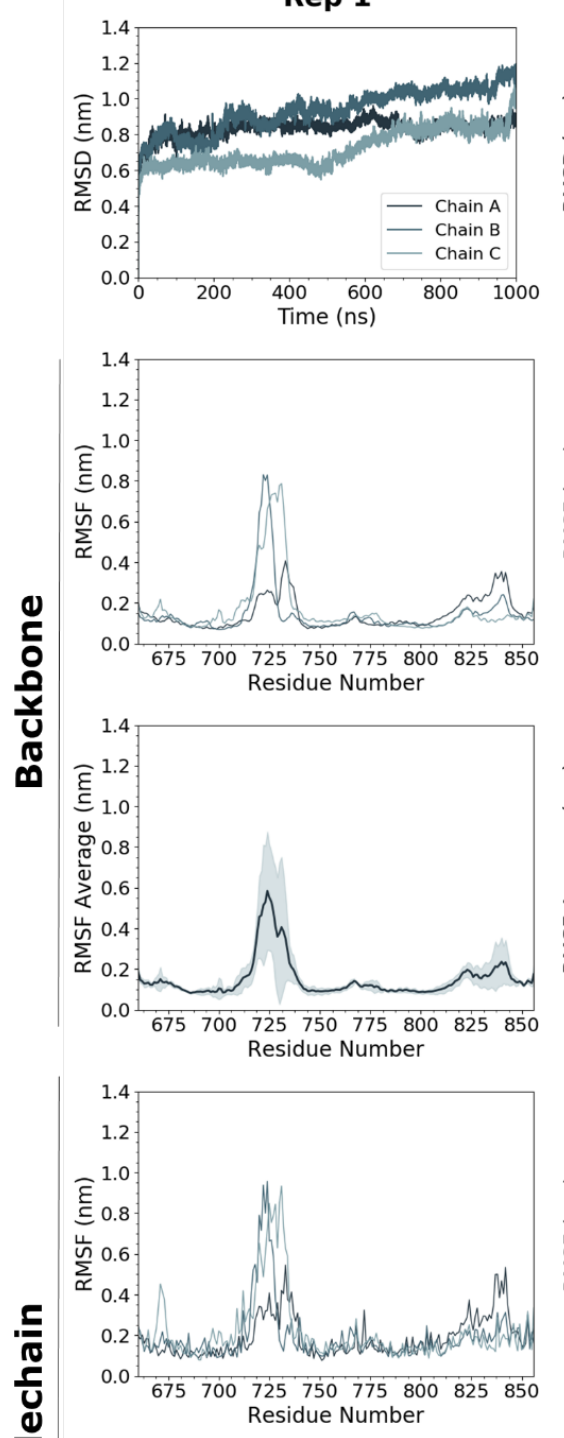

문

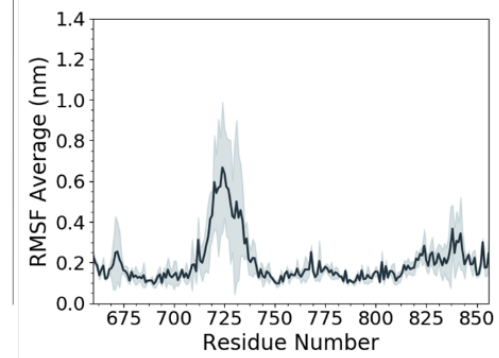

Rep 2
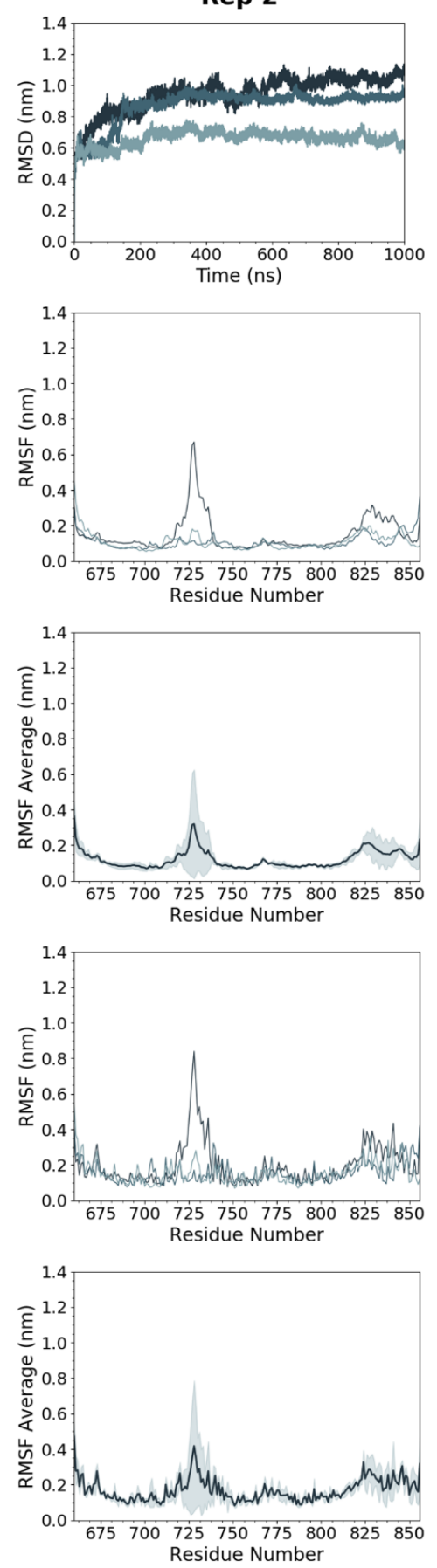

Rep 3
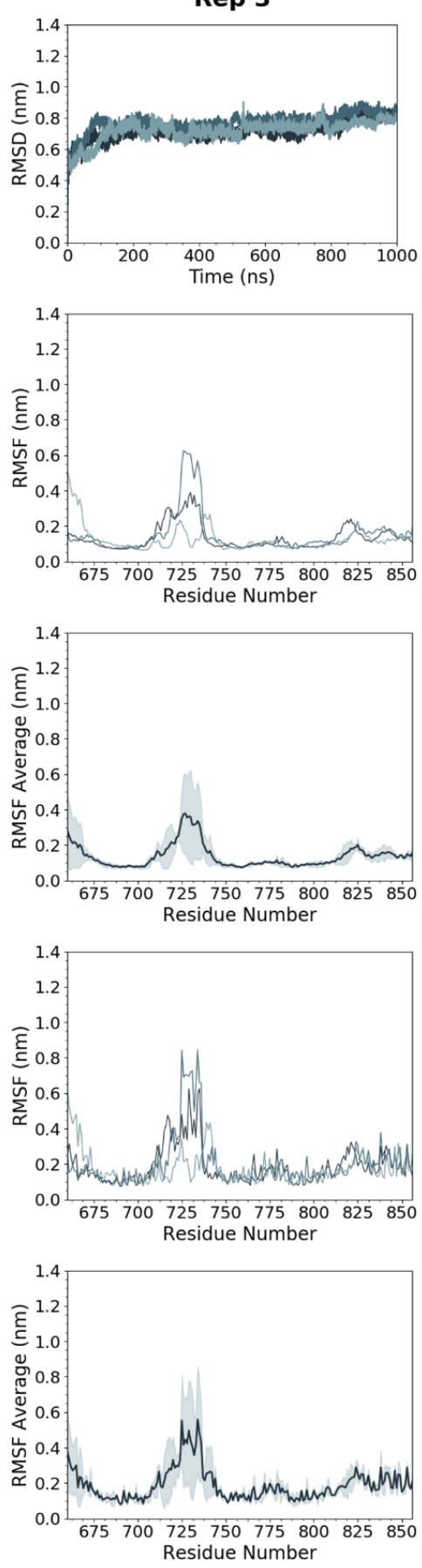

Figure S8. Root-mean-square deviation (RMSD) and root-mean-square fluctuation (RMSF) of backbone and sidechain atoms of gp41 MPER-TMD-CT. RMSD of backbone atoms is shown per chain, over the entire simulation time, for each replicate. Average per-residue RMSF over the last $100 \mathrm{~ns}$ of simulation time for both backbone and sidechain atoms is shown as both raw data per chain and as an average \pm standard deviation over all chains per replicate. 

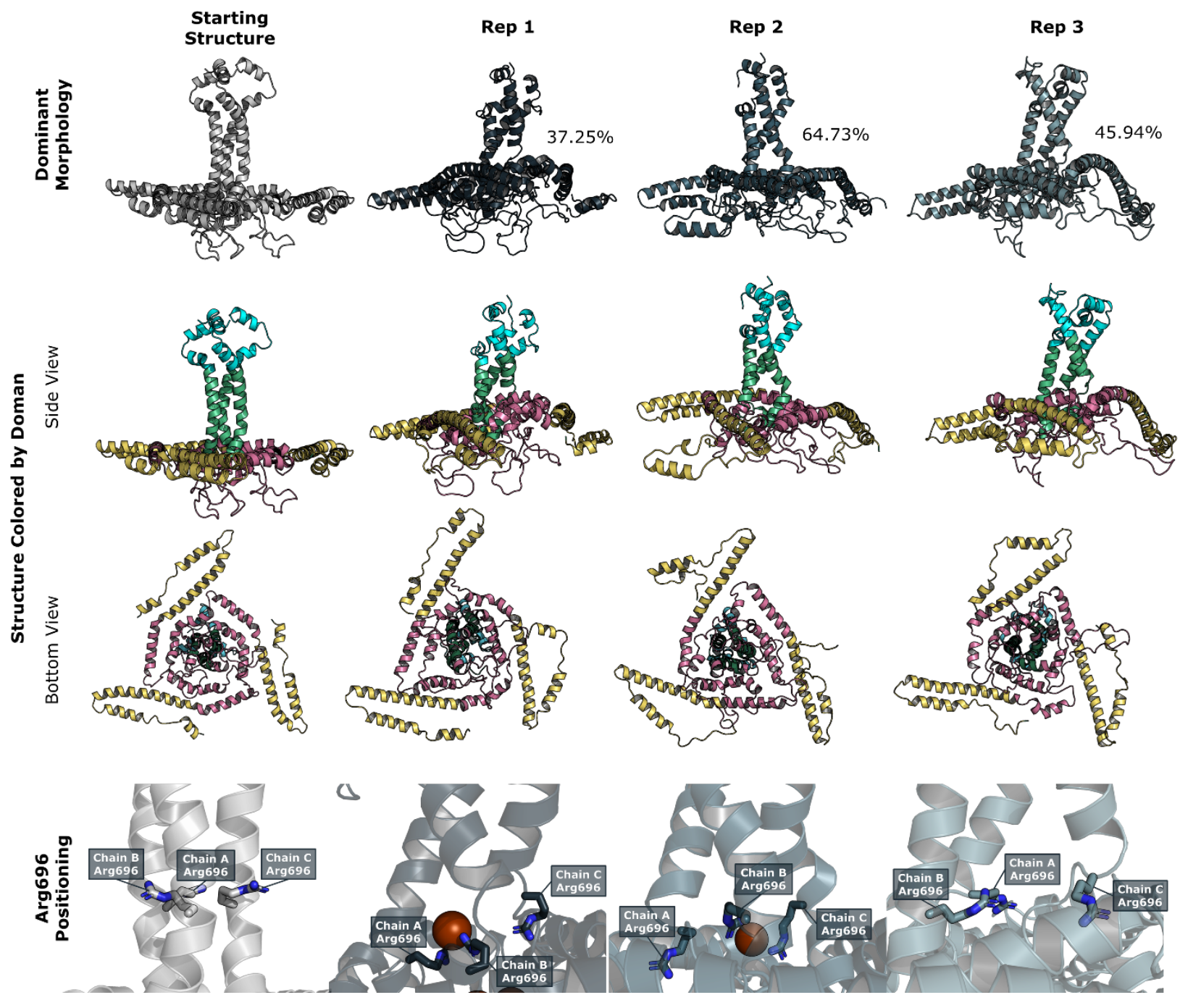

Figure S9. Dominant morphologies of gp41 MPER-TMD-CT trimer. Starting structure and each replicate dominant morphology, selected as the center structure of the largest cluster is shown, with associated percentage of cluster size. Clustering was performed over the last $100 \mathrm{~ns}$ with a backbone RMSD cut-off of $0.2 \mathrm{~nm}$. In the top row, MPERTMD-CT is colored by replicate. In the center, the gp41 MPER-TMD-CT trimer is colored based on domain and rotated in two viewpoints - cyan (MPER), green (TMD), pink (CT ${ }^{\text {LP2 }}$ ), and yellow (CT ${ }^{\text {LP3-LP1 }}$ ). Finally, a zoom-in of R696 positioning in the TMD with R696 shown as sticks colored by atom type. Chlorine ion represented as orange sphere. 

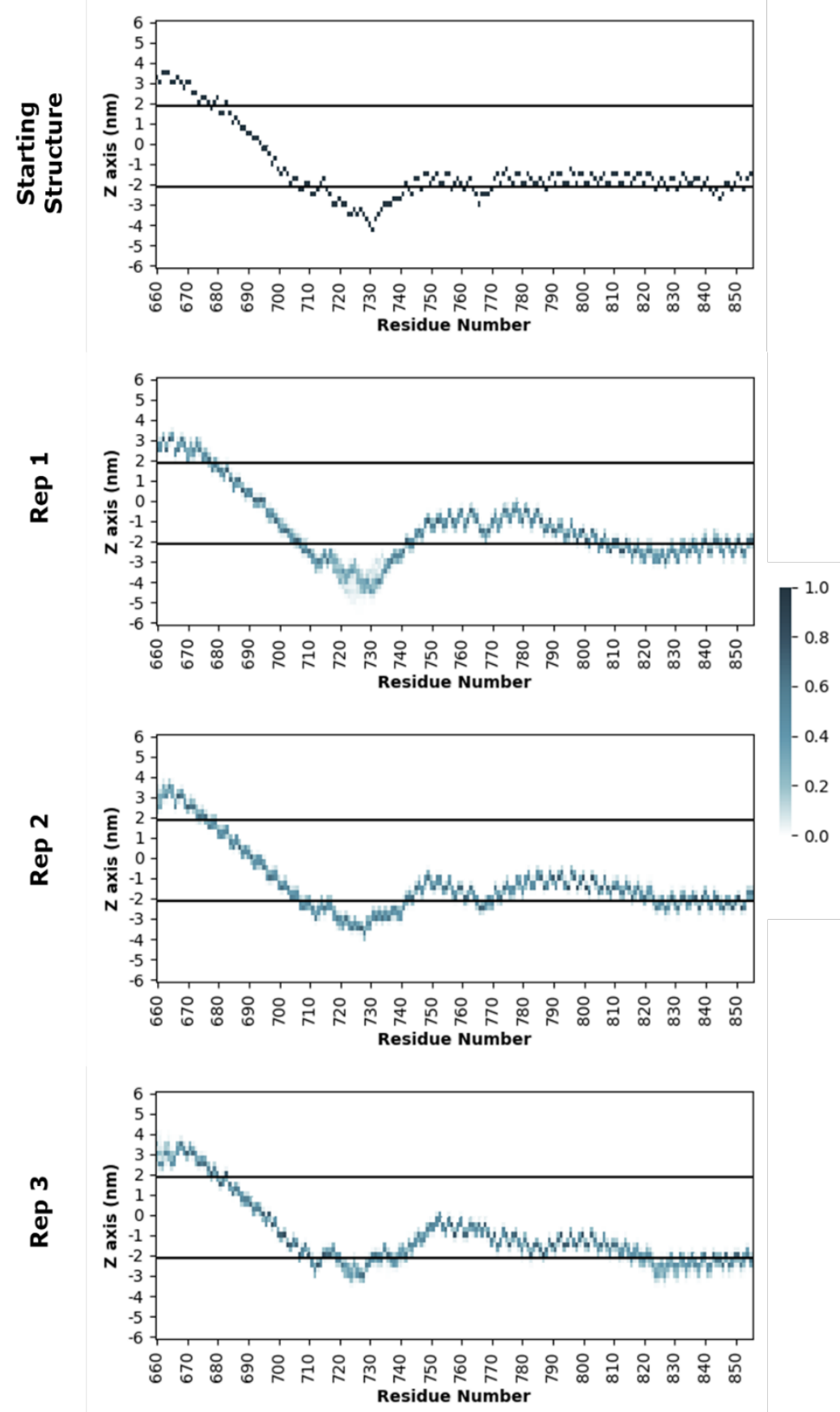

Figure S10. Center of mass (COM) positioning of gp41 MPER-TMD-CT trimer residues relevant to the membrane COM. Per-residue position averaged over all chains shown as a probability density (legend) in the z-coordinate over the last $100 \mathrm{~ns}$ of simulation. Black bars are provided at the approximate positions of the headgroups of the exofacial $(2 \mathrm{~nm})$ and cytofacial leaflet $(-2 \mathrm{~nm})$. 

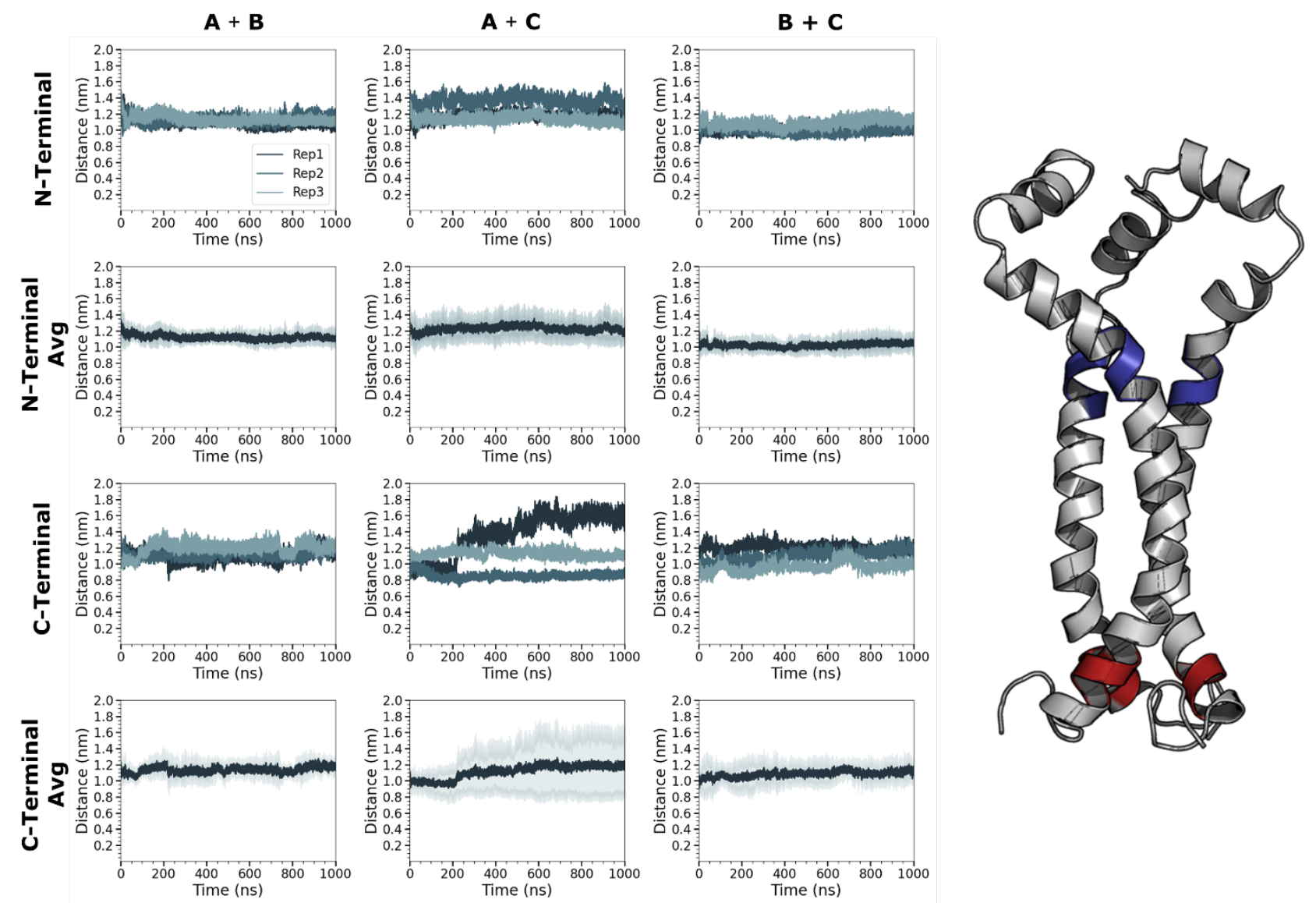

Figure S11. gp41 MPER-TMD-CT intra-trimer relative chain positioning over time for residues positioned at the headgroup of the exofacial leaflet ( $\mathrm{N}$-terminal) and cytofacial (C-terminal) leaflet. Data is shown as both raw timeseries data per replicate (replicates colored various shades of blue and average \pm standard deviation). N-terminal domains consisted of residues R683-1686 and the C-terminal domain consisted of residues L704-R707. Distance between each helix ( $A, B, C$ ) was calculated using the COM of each residue region relevant to the other. 

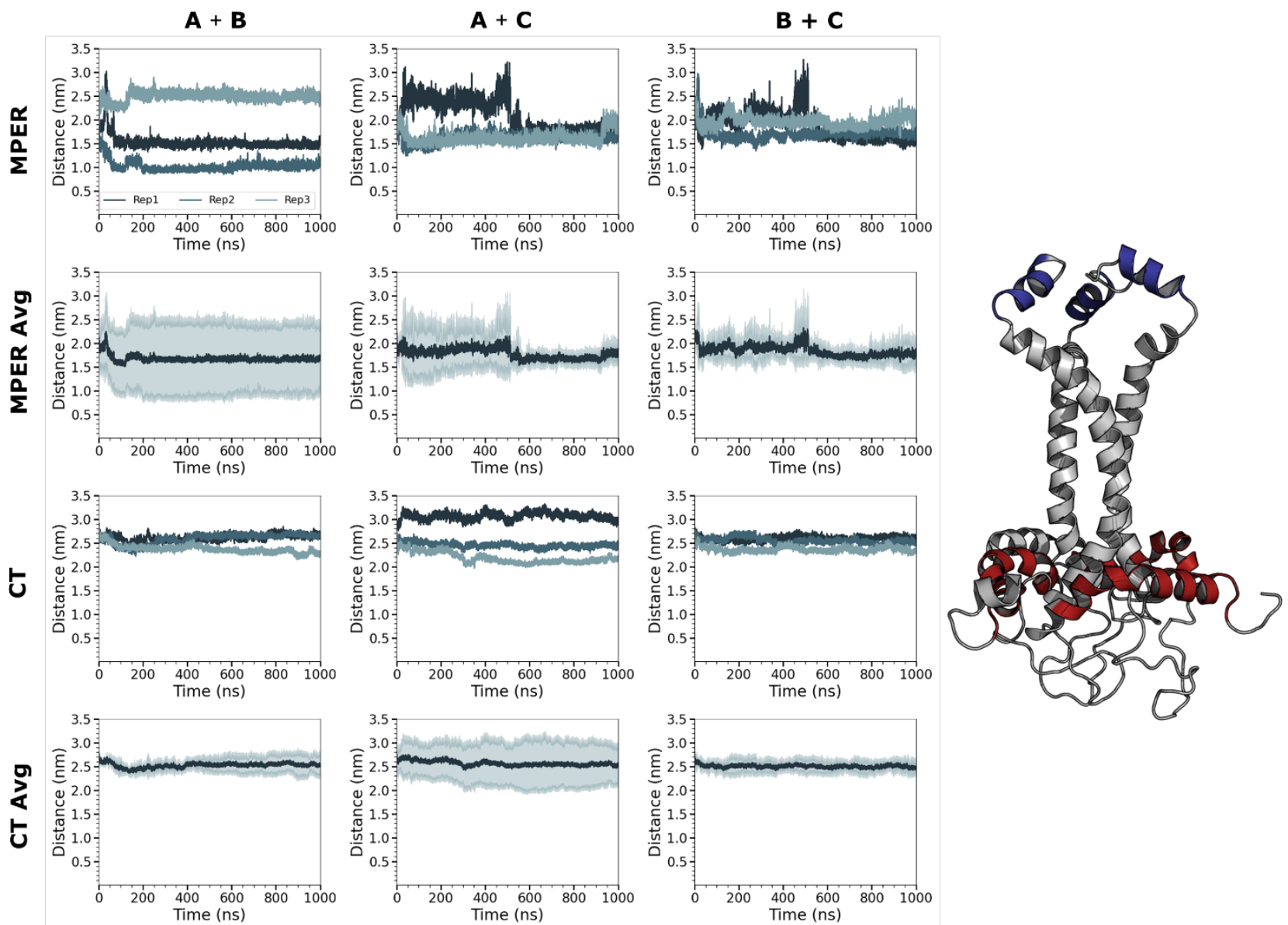

Figure S12. gp41 MPER-TMD-CT intra-trimer relative chain positioning over time for representative residues positioned in MPER and CT ${ }^{\mathrm{LP} 2}$ regions. Data is shown as both raw time-series data per replicate (replicates colored various shades of blue and average \pm standard deviation). Selected MPER region consisted of residues D664-W672 and selected $C T^{\text {LP2 }}$ region consists of residues D473-L765. Distance between each helix (A, B, C) was calculated using the COM of each residue region relevant to the other. 


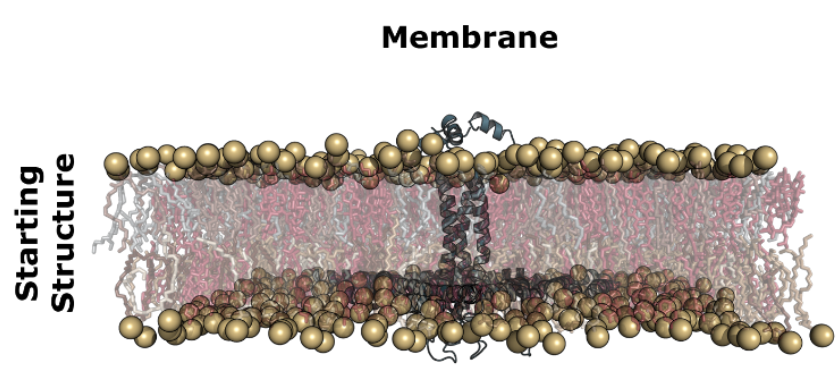

Water and Ions

within $<3.5 \AA$ of protein
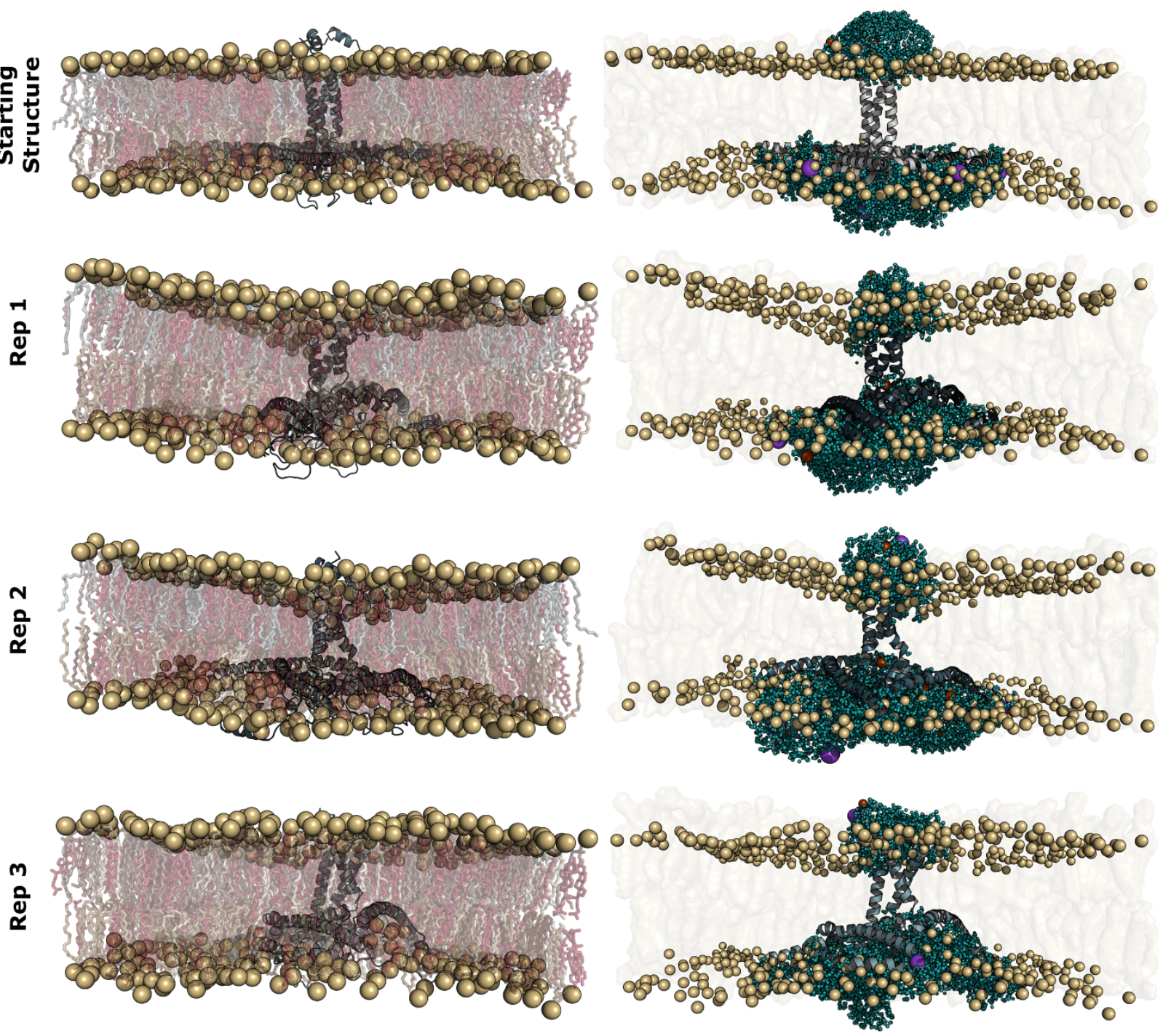

Figure S13. Membrane lipid, water, and ion placement for dominant morphologies of gp41 MPER-TMD-CT trimer. Dominant morphologies are calculated from RMSD clustering shown in Fig. S9. gp41 MPER-TMD-CT trimer is shown as cartoon, with membrane shown as stick (left) or surface(right). Phosphate atoms of lipid headgroups are shown as gold spheres. Water (teal spheres) and ions ( $\mathrm{K}^{+}$ions - purple spheres, $\mathrm{Cl}^{-}-$orange spheres) within $3.5 \AA$ of the MPER-TMD-CT trimer are shown (right). 

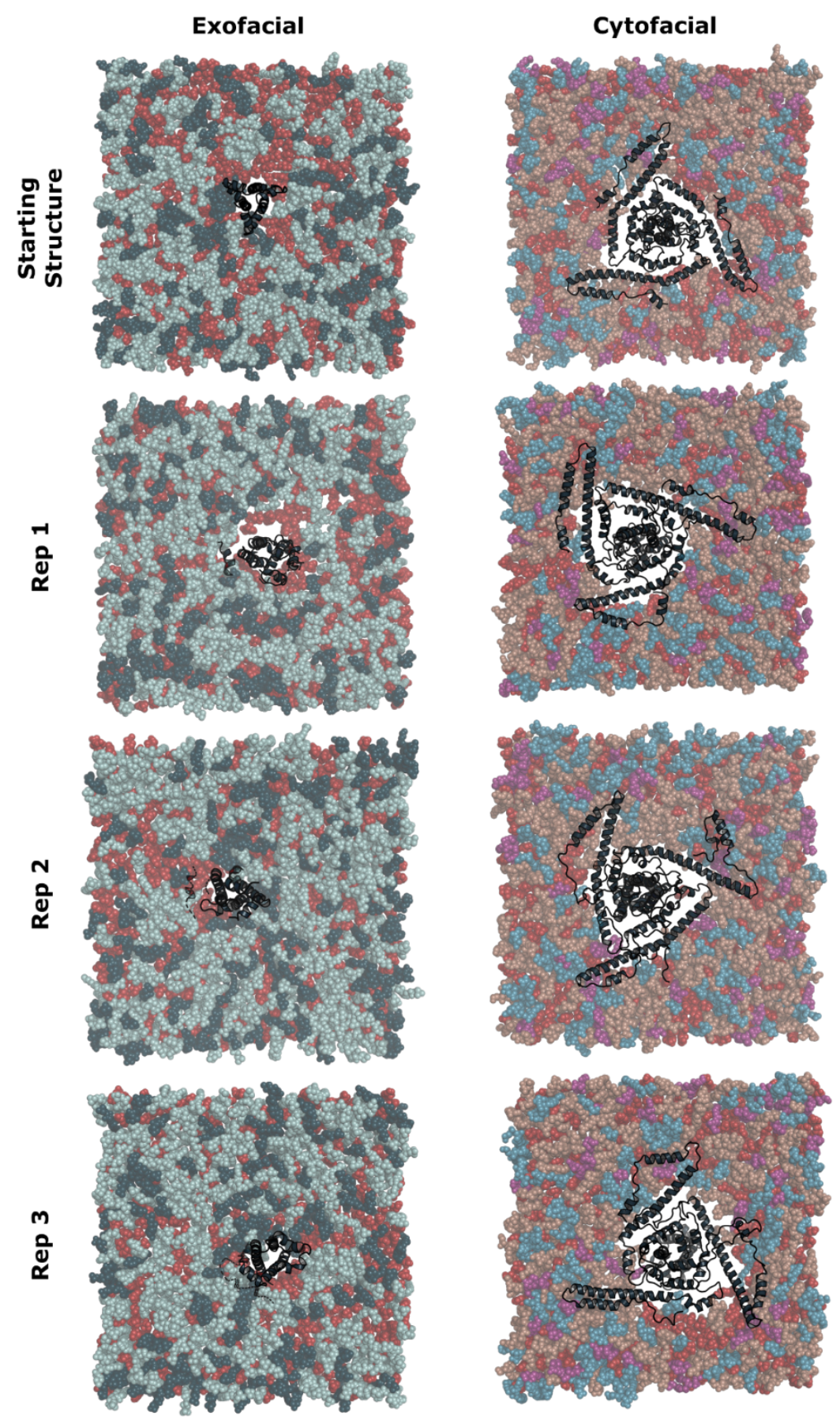

Figure S14. Distribution of exofacial and cytofacial lipids around the gp41 MPER-TMD-CT trimer for starting structure and each replicate dominant morphology. Dominant morphologies of each gp41 MPER-TMD-CT trimer are shown for each replicate and calculated as described in Fig. S9. Starting structure represents the energy minimized conformation of the system prior to equilibration. Each view is shown as a top-down of each leaflet. MPER-TMD-CT is shown as black cartoon and lipids are shown as spheres colored by lipid type (CHOL - red, dark teal - POPC, PSM - light teal, POPE - purple, SDPE - tan, SOPS - blue) 

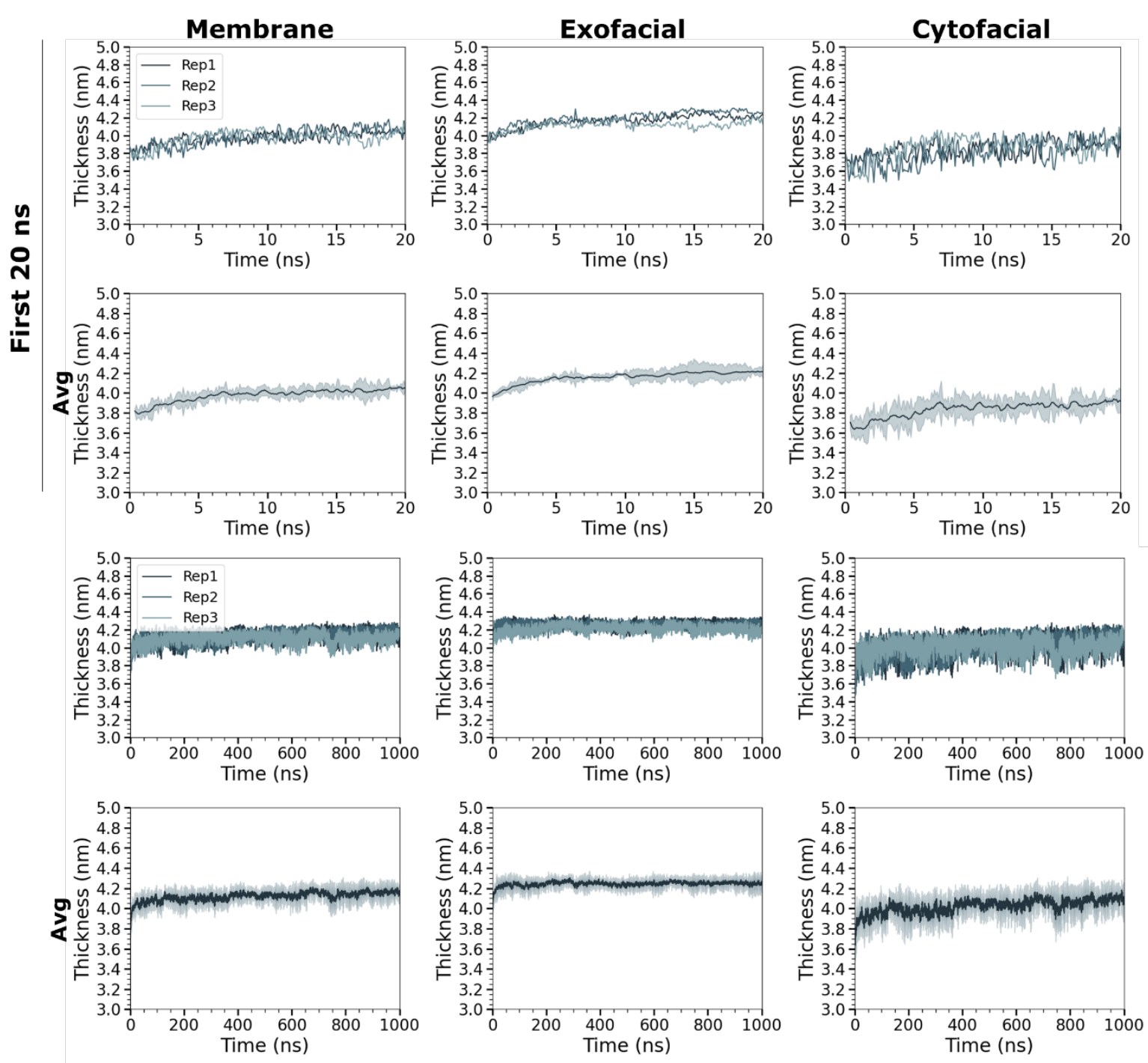

Figure S15. Membrane thickness over time per replicate. Full membrane, cytofacial leaflet, and exofacial leaflet thickness shown as raw data and as an average, calculated using FATSLiM thickness feature ${ }^{29}$, which estimates thickness for every lipid and averages values for both membrane and individual leaflets. 

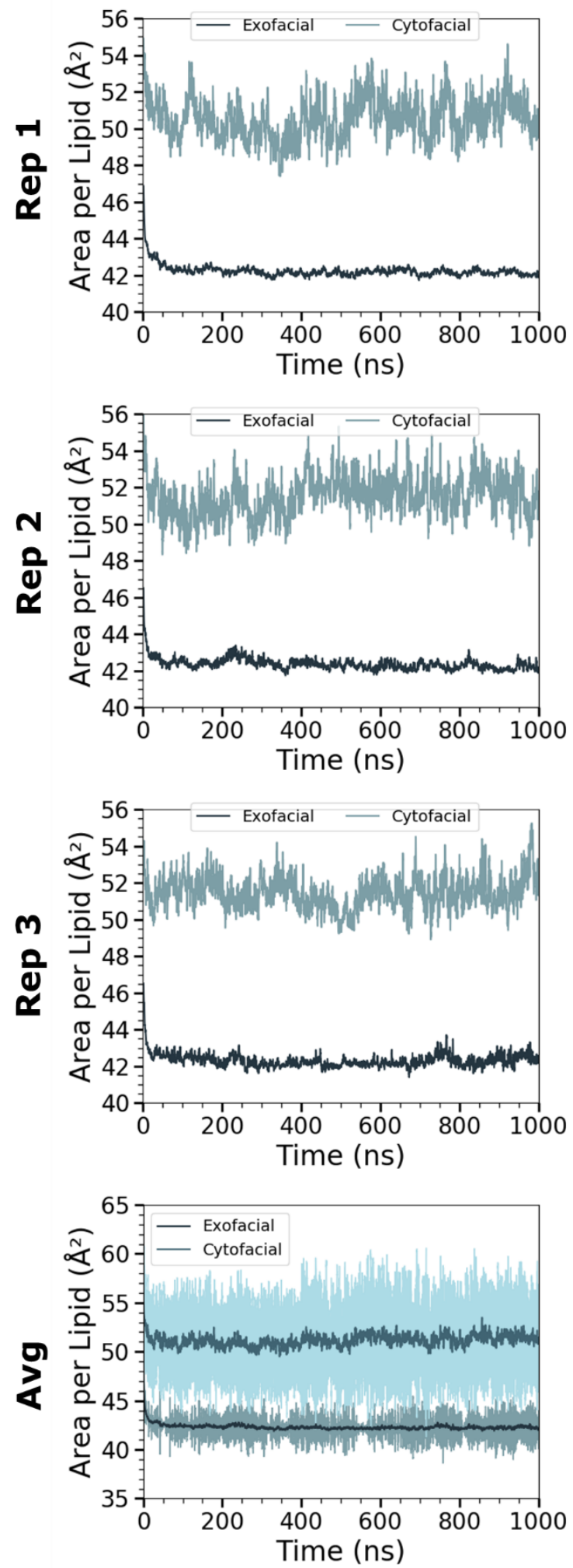

Figure S16. Area per Lipid (APL) over time for exofacial and cytofacial membrane leaflets. Data is shown per replicate and as an average \pm standard deviation over all replicates. 

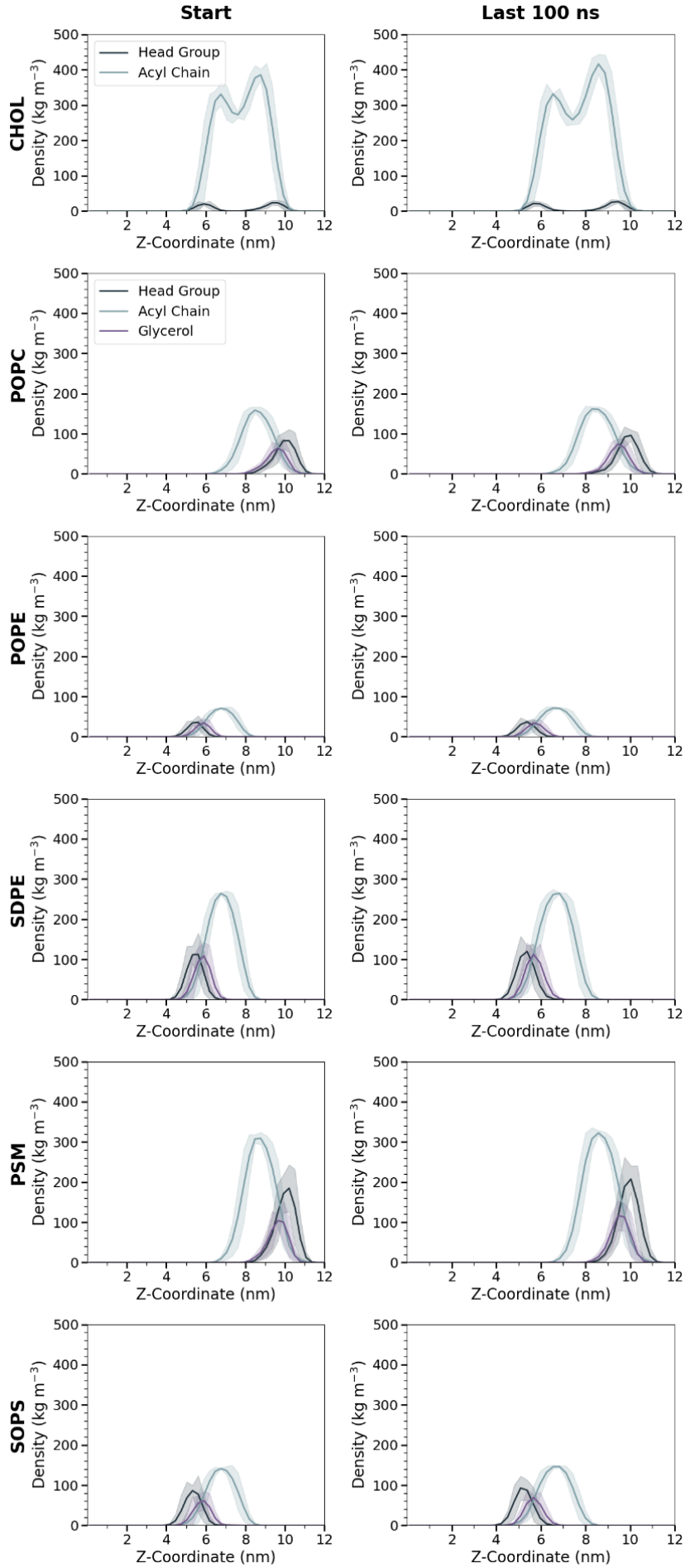

Figure S17. Lipid density in the z-axis. Each lipid was analyzed by their headgroup, glycerol, or acyl tail density position in the z-axis at the start of simulation (averaged over the first $20 \mathrm{~ns}$ ) or over the last $100 \mathrm{~ns}$ of simulation time. Each lipid atom group is averaged across three replicates with standard deviation shown. Exofacial lipid composition includes $\mathrm{CHOL}$, POPC, and PSM. Cytofacial lipid composition includes CHOL, POPE, SOPS, and SDPE. See methods for abbreviations. 

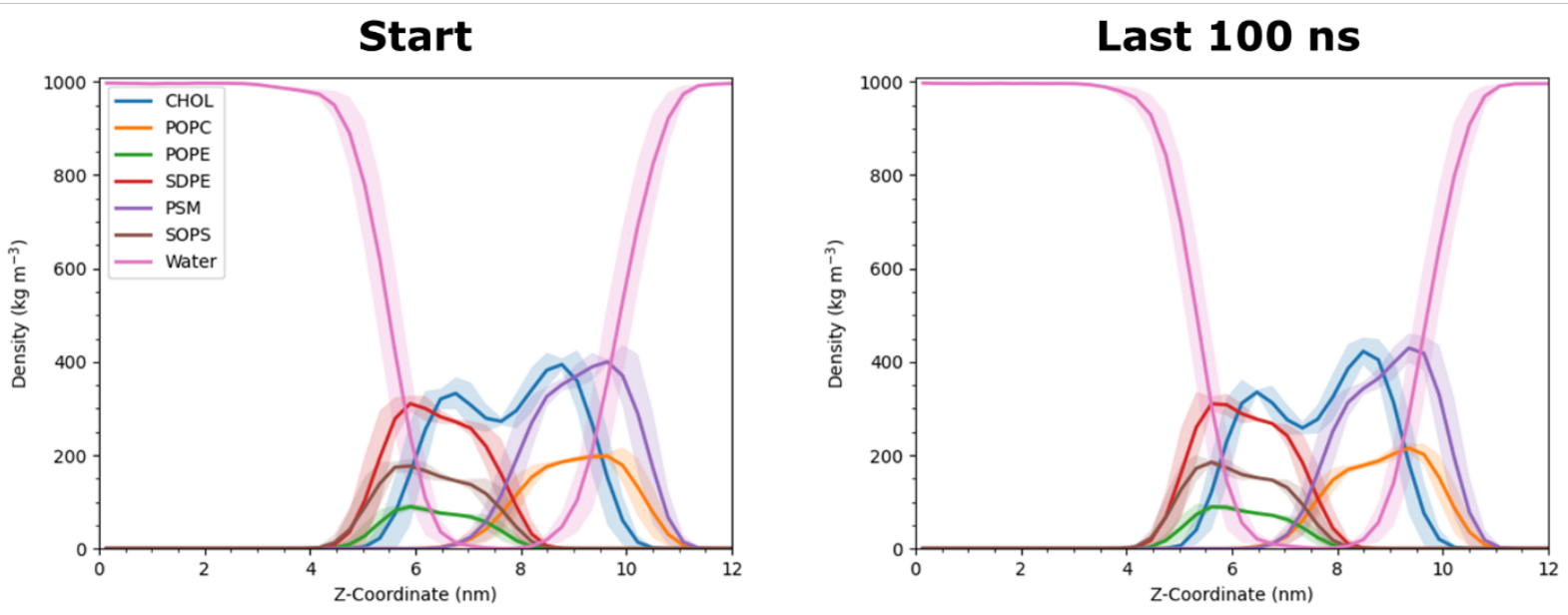

Figure S18. Lipid density in the z-axis. Each full lipid structure and water was analyzed for density position in the zaxis at the start of simulation (averaged over the first $20 \mathrm{~ns}$ ) or over the last $100 \mathrm{~ns}$ of simulation time. Each lipid atom group is averaged across three replicates with standard deviation shown. Exofacial lipid composition includes CHOL, POPC, and PSM. Cytofacial lipid composition includes CHOL, POPE, SOPS, and SDPE. See Table S1 for abbreviations. 

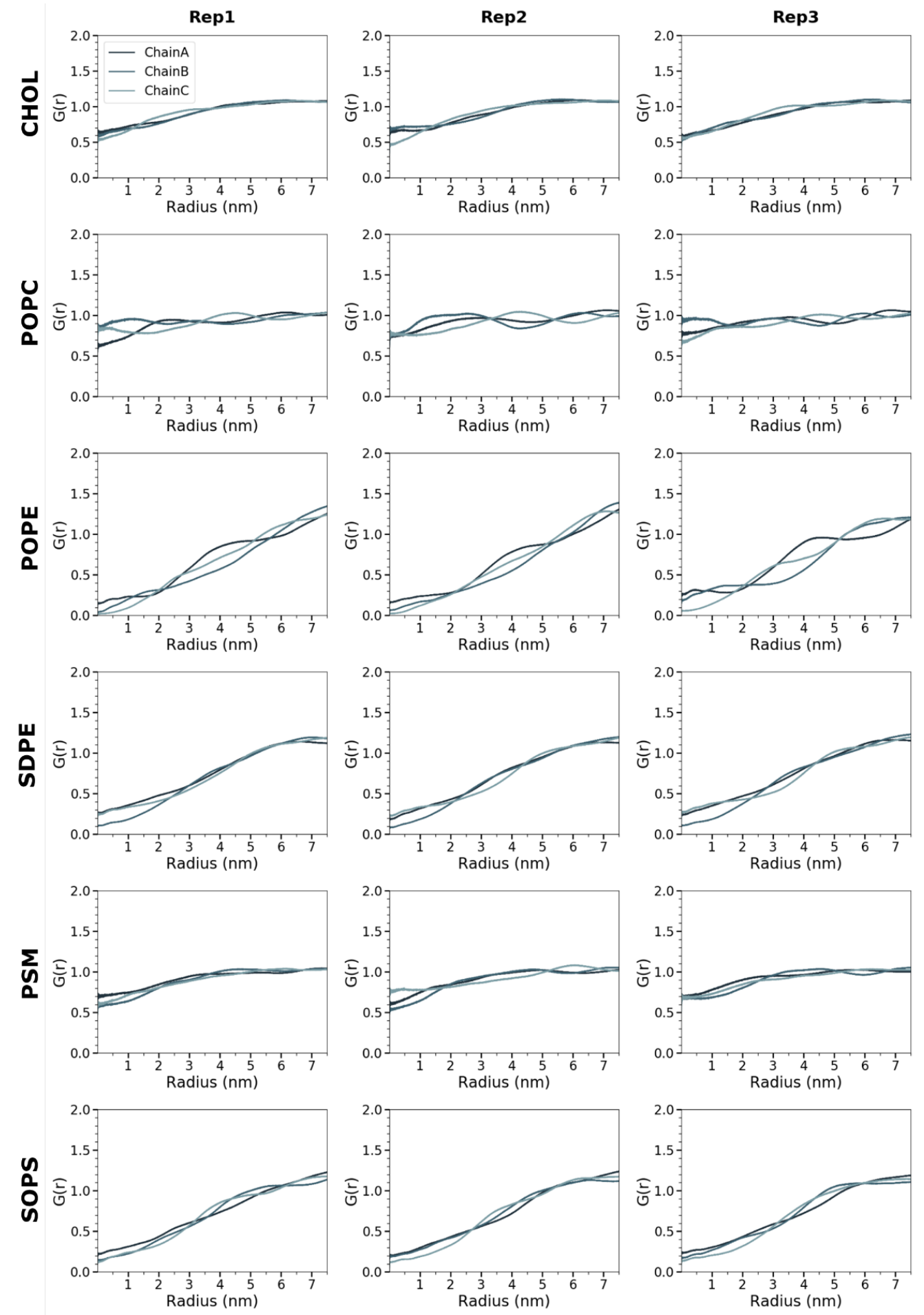

Figure S19. Radial distribution function (RDF) lipid analysis over the first $100 \mathrm{~ns}$ of gp41 MPER-TMD-CT simulation time. RDF of each lipid type is shown relative to each protein chain (colored shades of blue - A, B, C). Exofacial lipid composition includes $\mathrm{CHOL}$, POPC, PSM. Cytofacial lipid composition includes CHOL, POPE, SOPS, SDPE. 

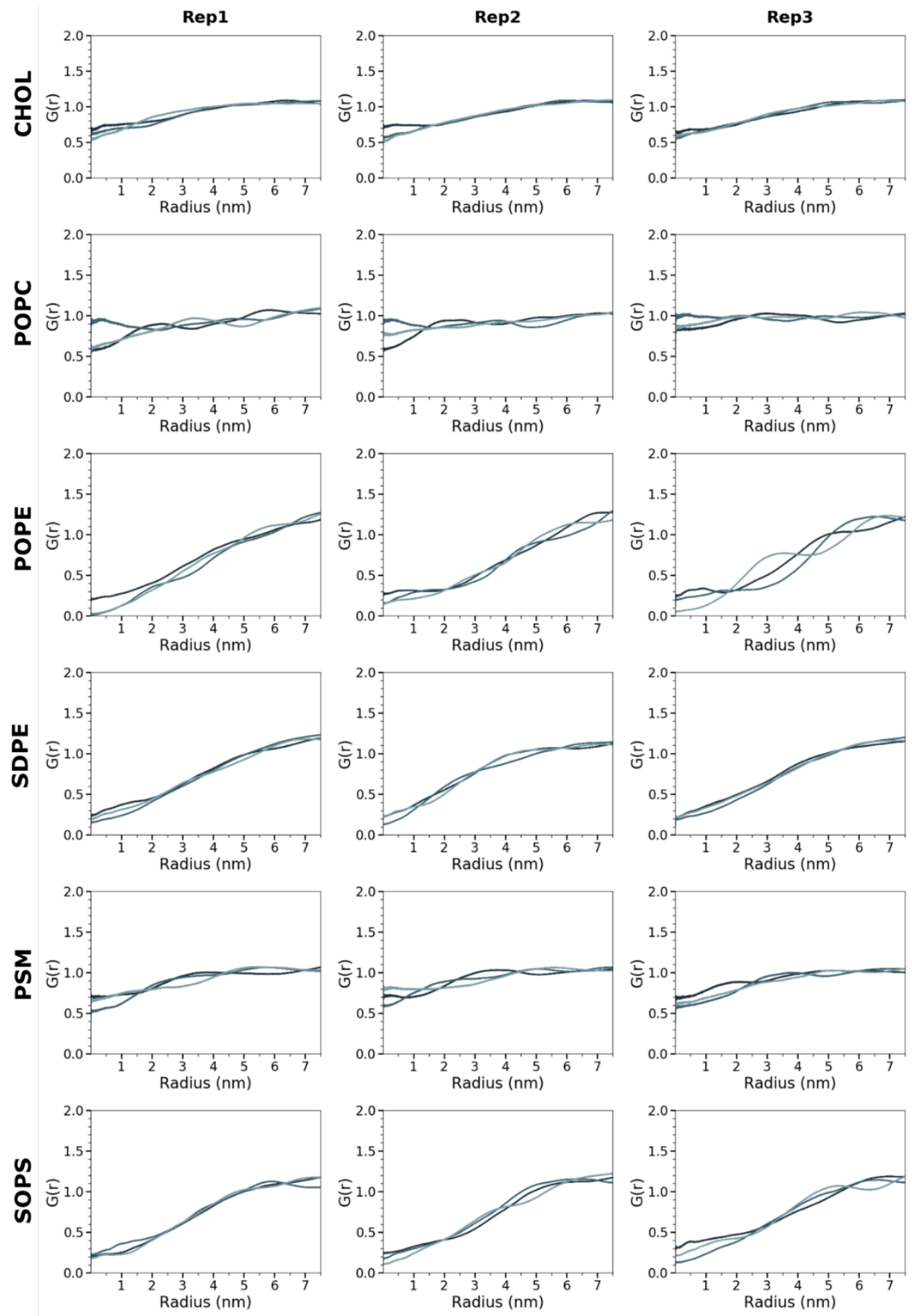

Figure S20. RDF lipid analysis over the last $100 \mathrm{~ns}$ of gp41 MPER-TMD-CT simulation time. RDF of each lipid type is shown relative to each protein chain (colored shades of blue - A, B, C). Exofacial lipid composition includes CHOL, POPC, PSM. Cytofacial lipid composition includes CHOL, POPE, SOPS, SDPE. 

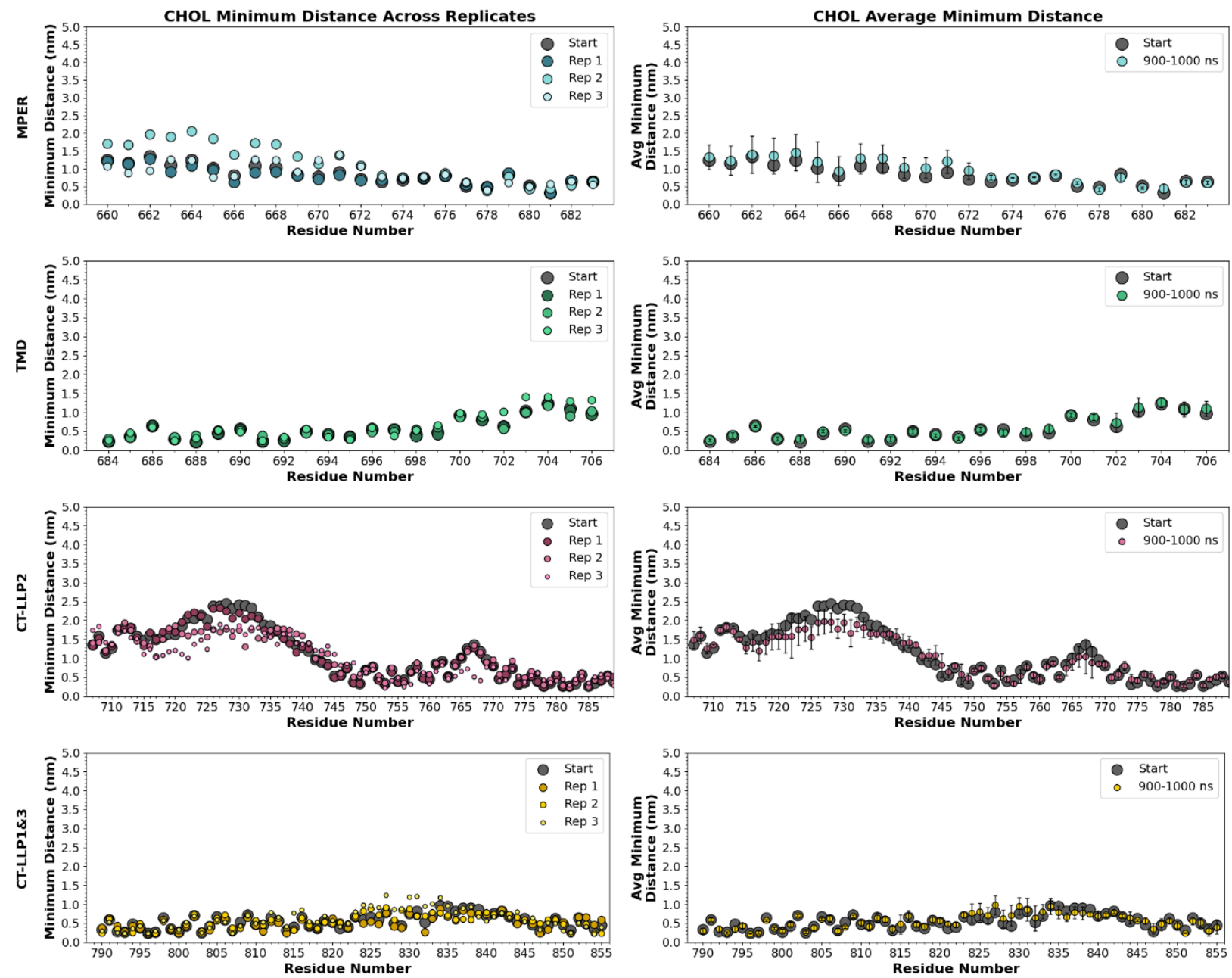

Figure S21. Average minimum distance per gp41 MPER-TMD-CT residue with cholesterol. Distances are an average of position of each atom of each residue for each helix over the last $100 \mathrm{~ns}$ of simulation time. Start distance and individual replicate data is shown (left) with average \pm standard deviation across three replicates is also shown (right). Each graph is colored based on gp41 trimer domain (cyan - MPER, green $-\mathrm{TMD}$, pink $-\mathrm{CT}^{\text {LLP2 }}$, yellow $-\mathrm{CT}^{\mathrm{LP} 3-}$ ${ }^{\text {LP1 } 1) .}$ 

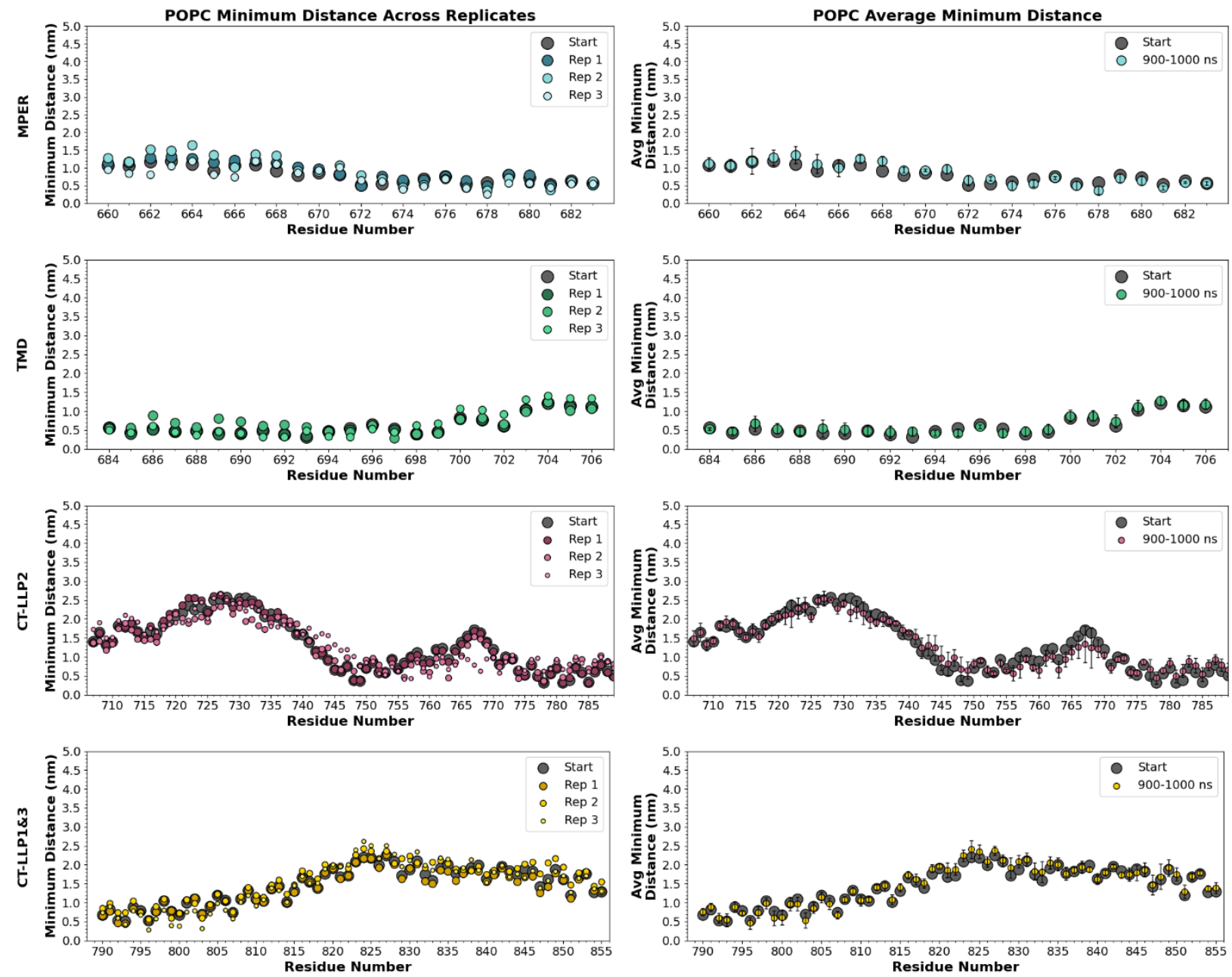

Figure S22. Average minimum distance per gp41 MPER-TMD-CT residue with POPC. Distances are an average of position of each atom of each residue for each helix over the last $100 \mathrm{~ns}$ of simulation time. Start distance and individual replicate data is shown (left) with average \pm standard deviation across three replicates is also shown (right). Each graph is colored based on gp41 trimer domain (cyan - MPER, green $-\mathrm{TMD}$, pink $-\mathrm{CT}^{\mathrm{LP} 2}$, yellow $-\mathrm{CT}^{\mathrm{LP} 3-}$ ${ }^{\text {LP1 } 1 \text { ). }}$ 

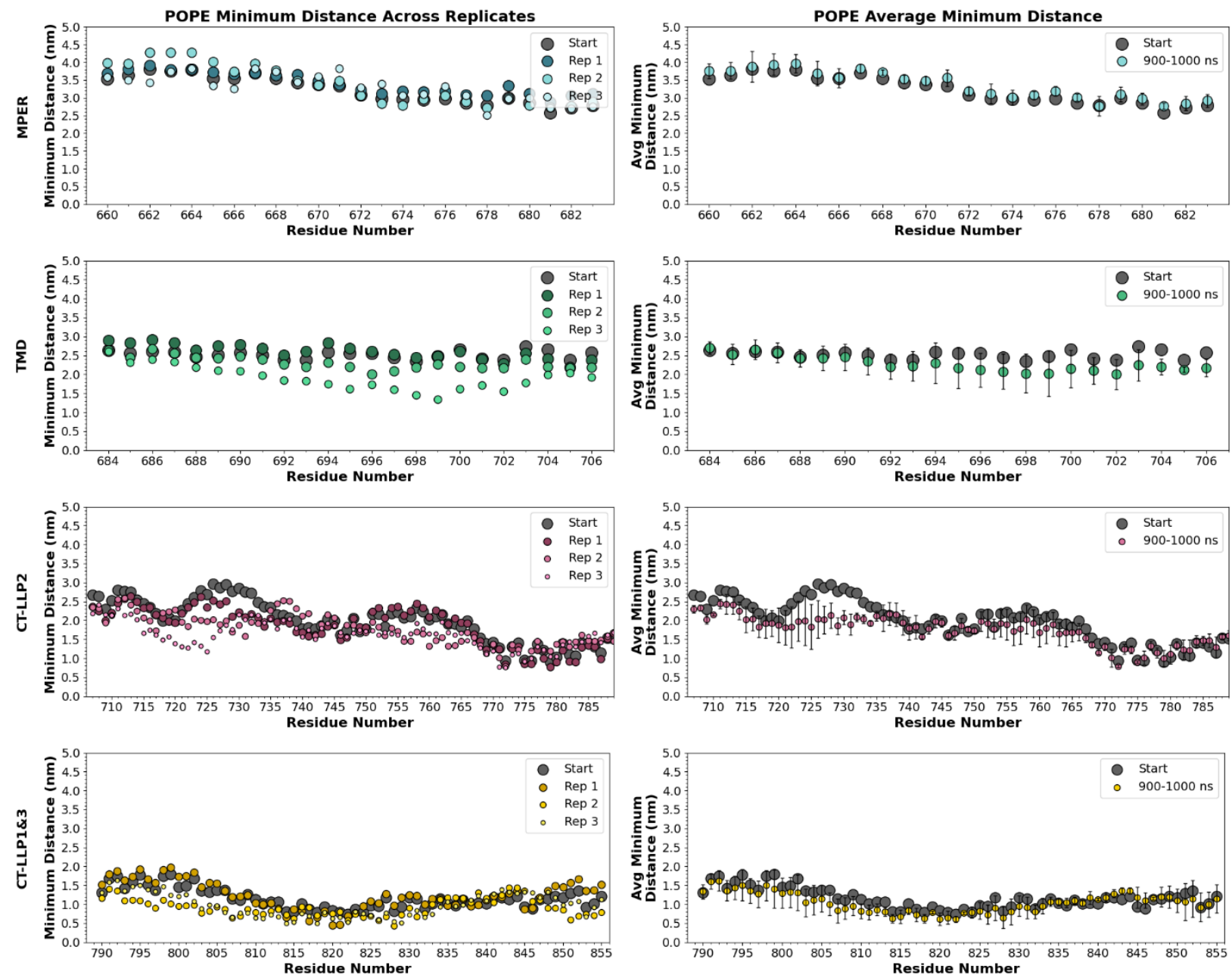

Figure S23. Average minimum distance per gp41 MPER-TMD-CT residue with POPE. Distances are an average of position of each atom of each residue for each helix over the last $100 \mathrm{~ns}$ of simulation time. Start distance and individual replicate data is shown (left) with average \pm standard deviation across three replicates is also shown (right). Each graph is colored based on gp41 trimer domain (cyan - MPER, green $-\mathrm{TMD}$, pink $-\mathrm{CT}^{\mathrm{LP} 2}$, yellow $-\mathrm{CT}^{\mathrm{LP} 3-}$ ${ }^{\text {LP1 } 1) .}$ 

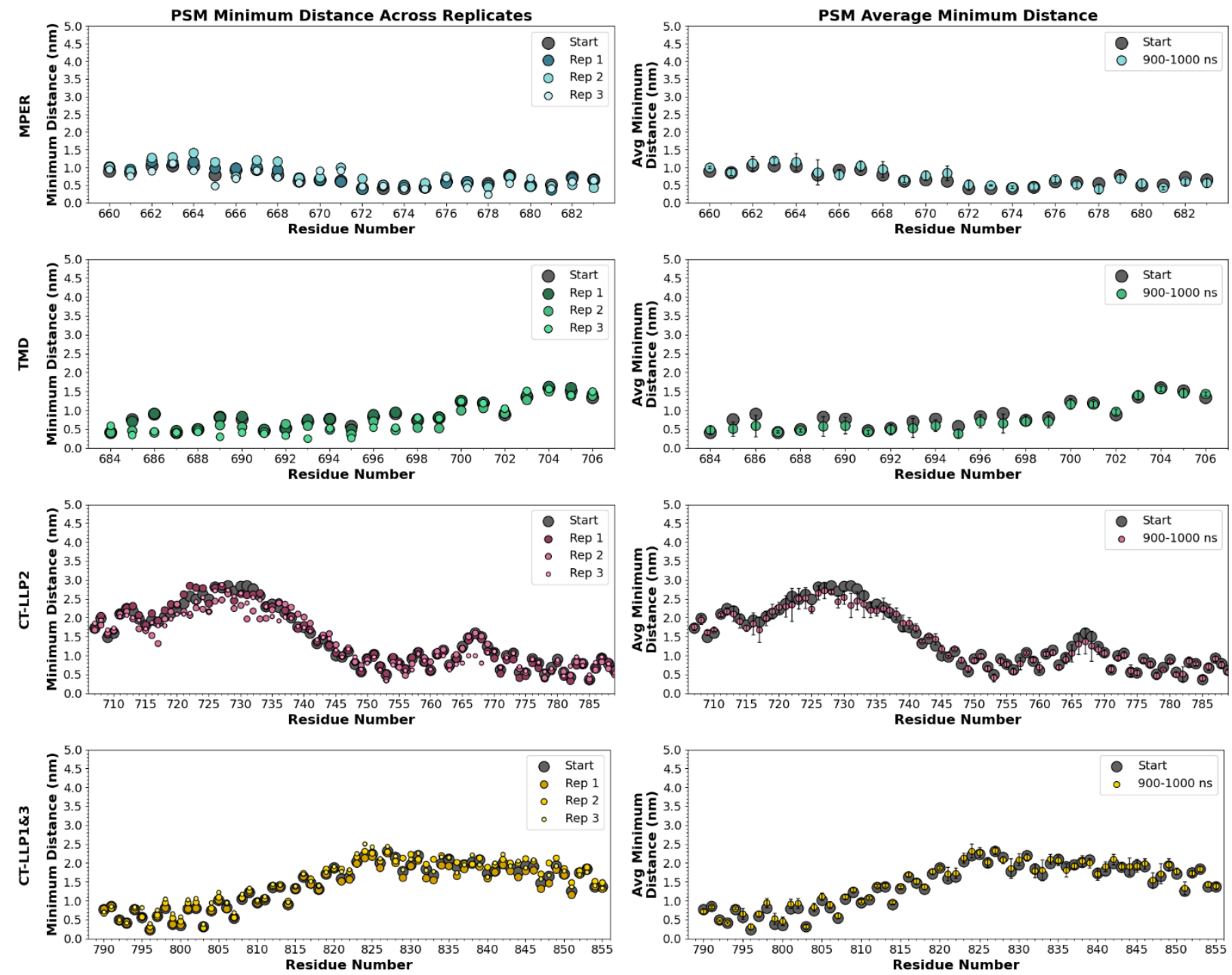

Figure S24. Average minimum distance per gp41 MPER-TMD-CT residue with PSM. Distances are an average of position of each atom of each residue for each helix over the last 100 ns of simulation time. Start distance and individual replicate data is shown (left) with average \pm standard deviation across three replicates is also shown (right). Each graph is colored based on gp41 trimer domain (cyan - MPER, green - TMD, pink $-\mathrm{CT}^{\mathrm{LLP2}}$, yellow $-\mathrm{CT}^{\mathrm{LLP} 3-}$ ${ }^{\mathrm{LLP}}{ }^{\text {). }}$. 

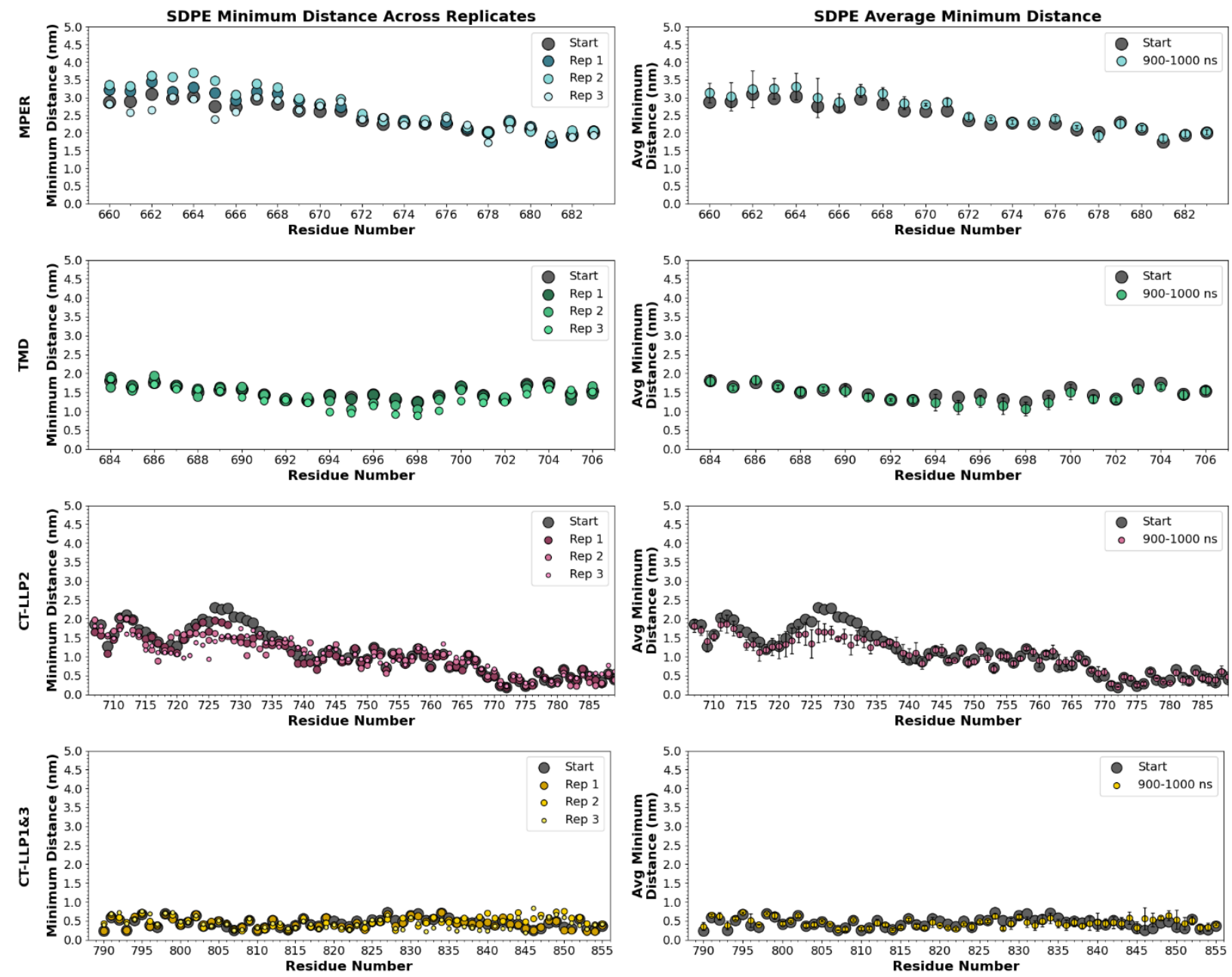

Figure S25. Average minimum distance per gp41 MPER-TMD-CT residue with SDPE. Distances are an average of position of each atom of each residue for each helix over the last $100 \mathrm{~ns}$ of simulation time. Start distance and individual replicate data is shown (left) with average \pm standard deviation across three replicates is also shown (right). Each graph is colored based on gp41 trimer domain (cyan - MPER, green $-\mathrm{TMD}$, pink $-\mathrm{CT}^{\mathrm{LP} 2}$, yellow $-\mathrm{CT}^{\mathrm{LP} 3-}$ ${ }^{\text {LP1 } 1 \text { ). }}$ 

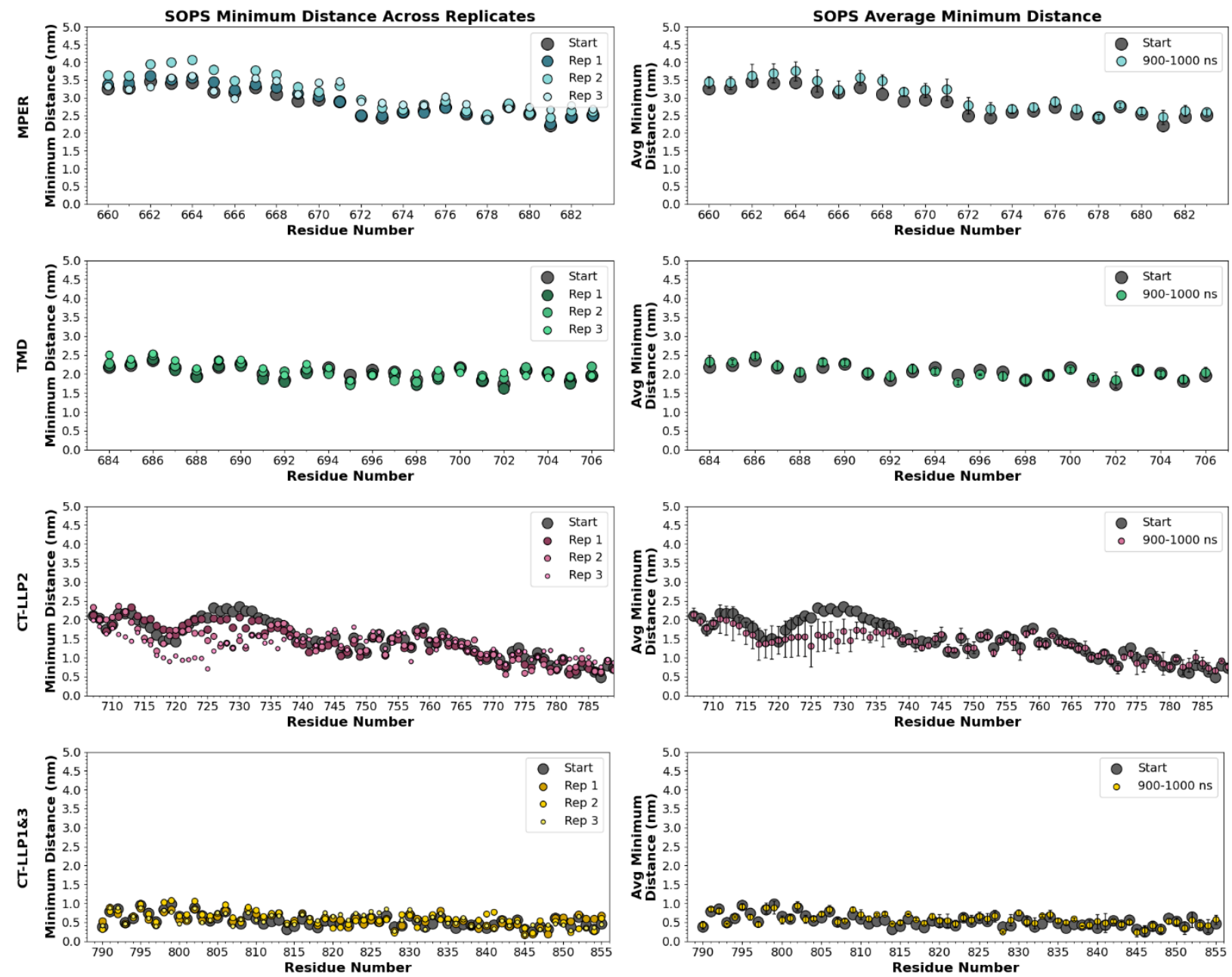

Figure S26. Average minimum distance per gp41 MPER-TMD-CT residue with SOPS. Distances are an average of position of each atom of each residue for each helix over the last $100 \mathrm{~ns}$ of simulation time. Start distance and individual replicate data is shown (left) with average \pm standard deviation across three replicates is also shown (right). Each graph is colored based on gp41 trimer domain (cyan - MPER, green - TMD, pink $-\mathrm{CT}^{\mathrm{LLP2}}$, yellow $-\mathrm{CT}^{\mathrm{LP} 3-}$ ${ }^{\text {LP1 } 1) .}$ 

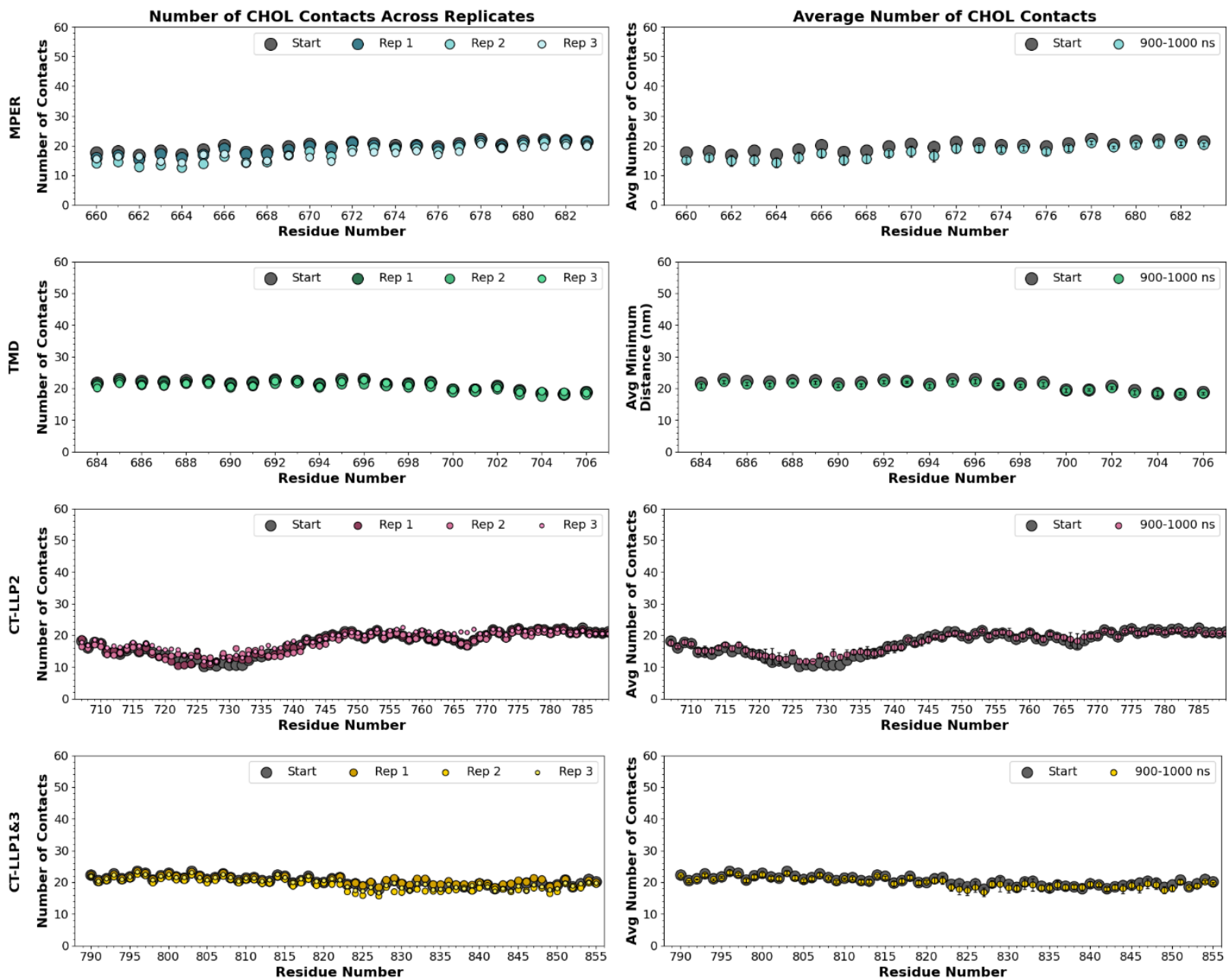

Figure S27. Normalized count of residue-cholesterol interactions over the last 100 ns of simulation time. Contacts were normalized by dividing by the total number of cholesterol lipids in the system. Start distance and individual replicate data is shown (left) with average \pm standard deviation across three replicates is also shown (right). Each graph is colored based on the MPER-TMD-CT trimer domain (cyan - MPER, green - TMD, pink $-\mathrm{CT}^{\mathrm{LLP2}}$, yellow - CT ${ }^{\mathrm{LLP} 3-}$ ${ }^{\mathrm{LLP}}{ }^{\text {). }}$ 

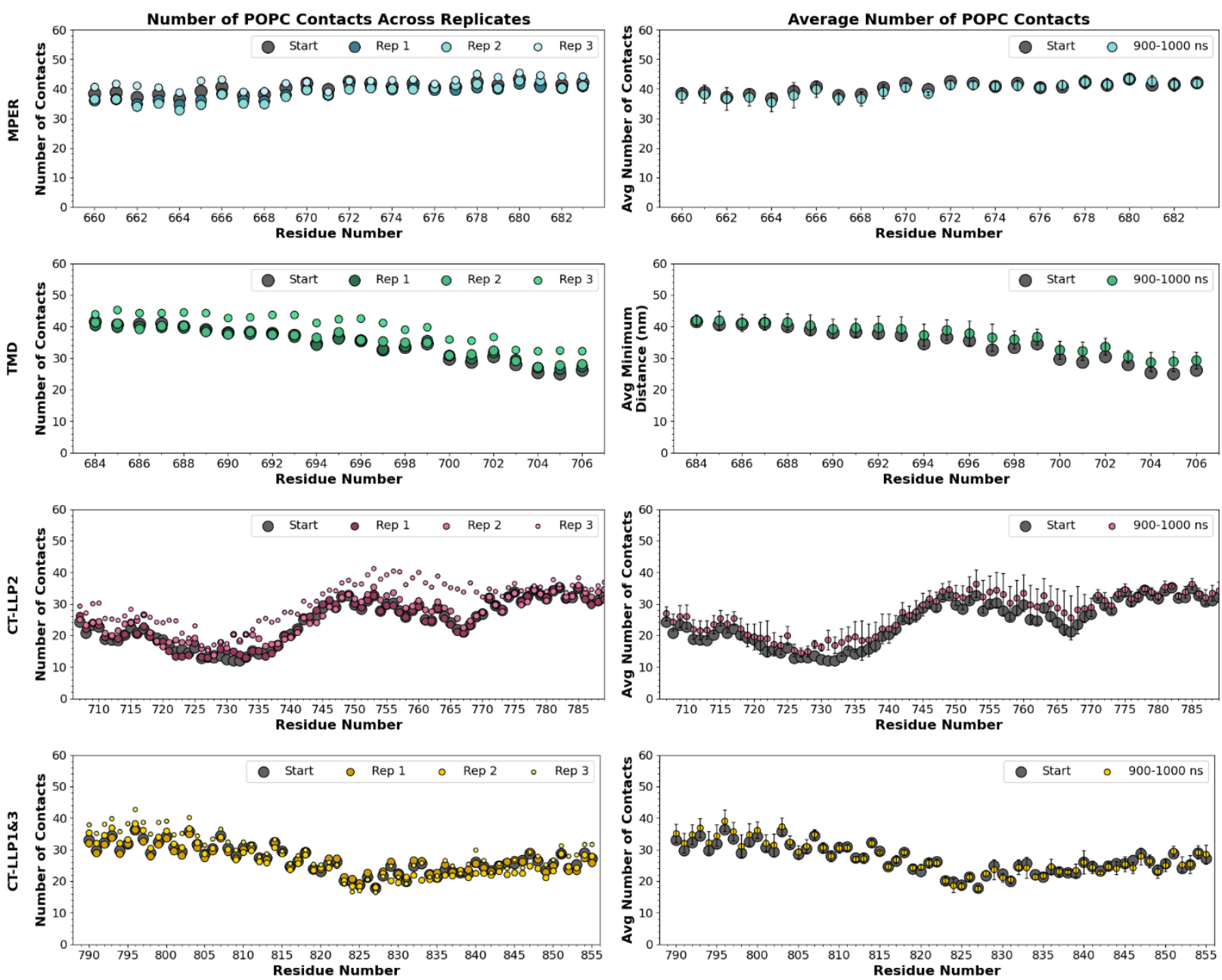

Figure S28. Normalized count of residue-POPC interactions over the last $100 \mathrm{~ns}$ of simulation time. Contacts were normalized by dividing by the total number of cholesterol lipids in the system. Start distance and individual replicate data is shown (left) with average \pm standard deviation across three replicates is also shown (right). Each graph is colored based on the MPER-TMD-CT trimer domain (cyan - MPER, green - TMD, pink $-\mathrm{CT}^{\mathrm{LLP} 2}$, yellow $-\mathrm{CT}^{\mathrm{LP} 3-\mathrm{LP} 1}$ ). 

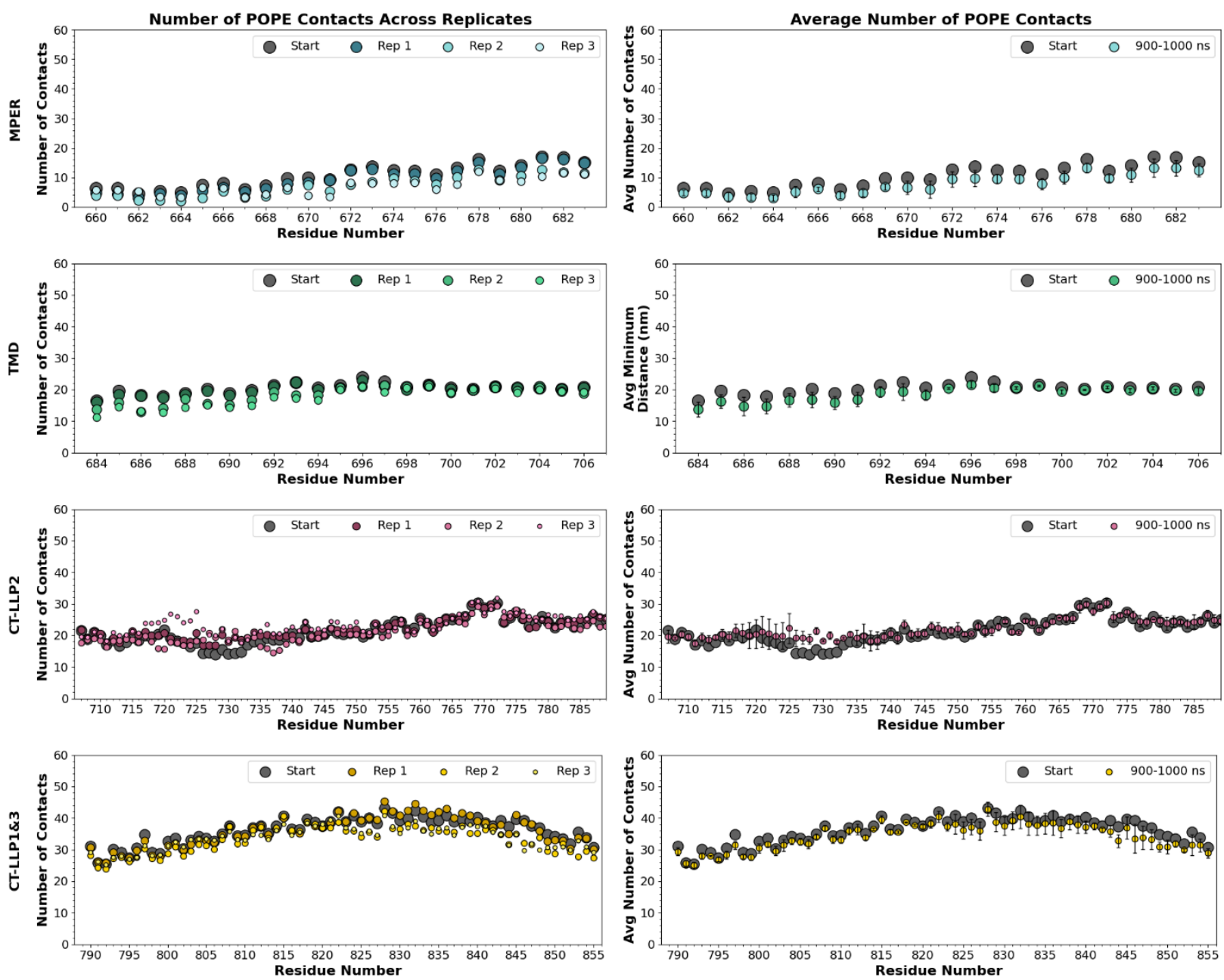

Figure S29. Normalized count of residue-POPE interactions over the last 100 ns of simulation time. Contacts were normalized by dividing by the total number of cholesterol lipids in the system. Start distance and individual replicate data is shown (left) with average \pm standard deviation across three replicates is also shown (right). Each graph is colored based on the MPER-TMD-CT trimer domain (cyan - MPER, green - TMD, pink - CT ${ }^{\text {LP2 }}$, yellow - CT ${ }^{\text {LP3-LLP1 }}$ ). 

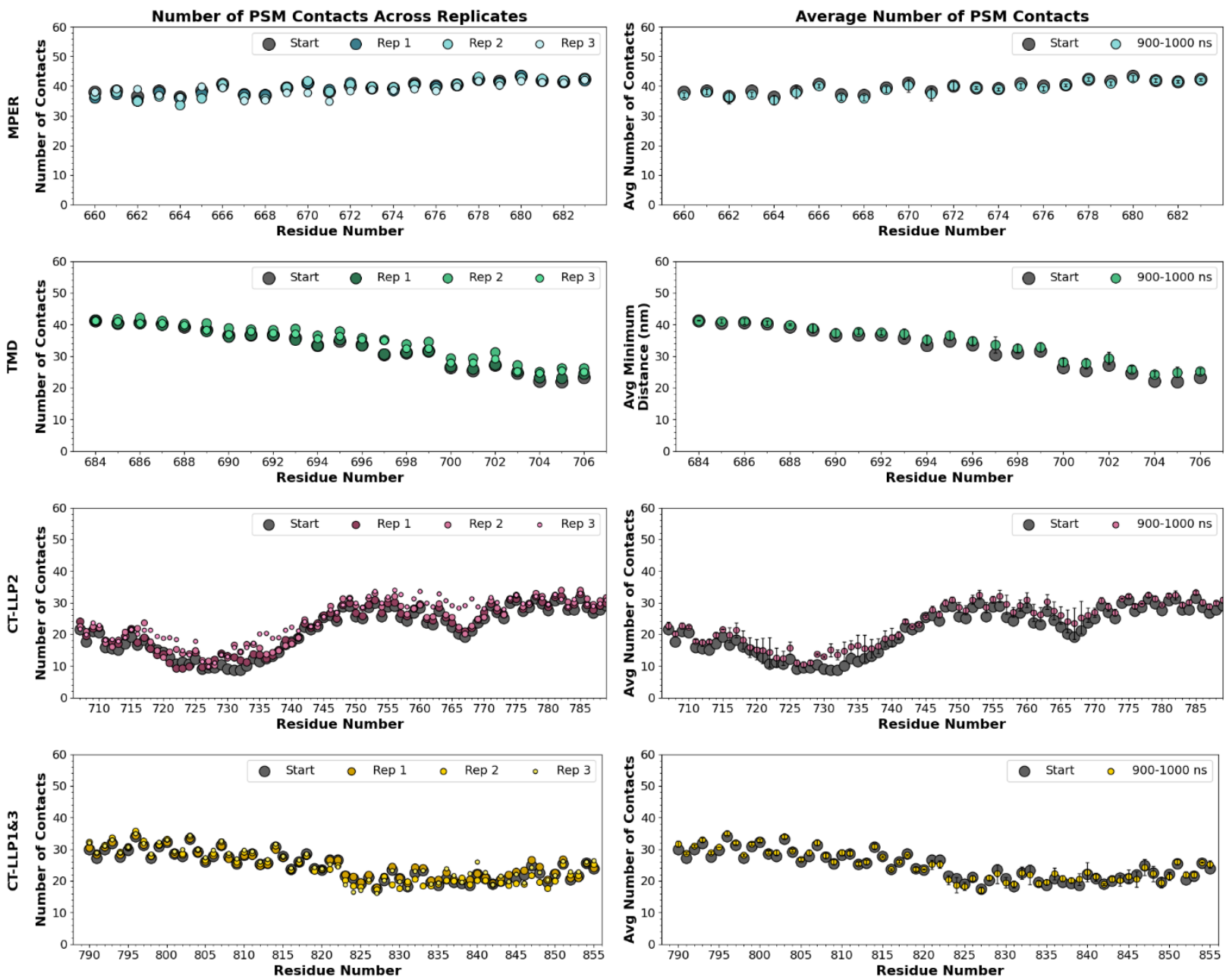

Figure S30. Normalized count of residue-PSM interactions over the last $100 \mathrm{~ns}$ of simulation time. Contacts were normalized by dividing by the total number of cholesterol lipids in the system. Start distance and individual replicate data is shown (left) with average \pm standard deviation across three replicates is also shown (right). Each graph is colored based on the MPER-TMD-CT trimer domain (cyan - MPER, green - TMD, pink - CT ${ }^{\text {LLP2 }}$, yellow - CT ${ }^{\text {LLP3-LLP1 }}$ ). 

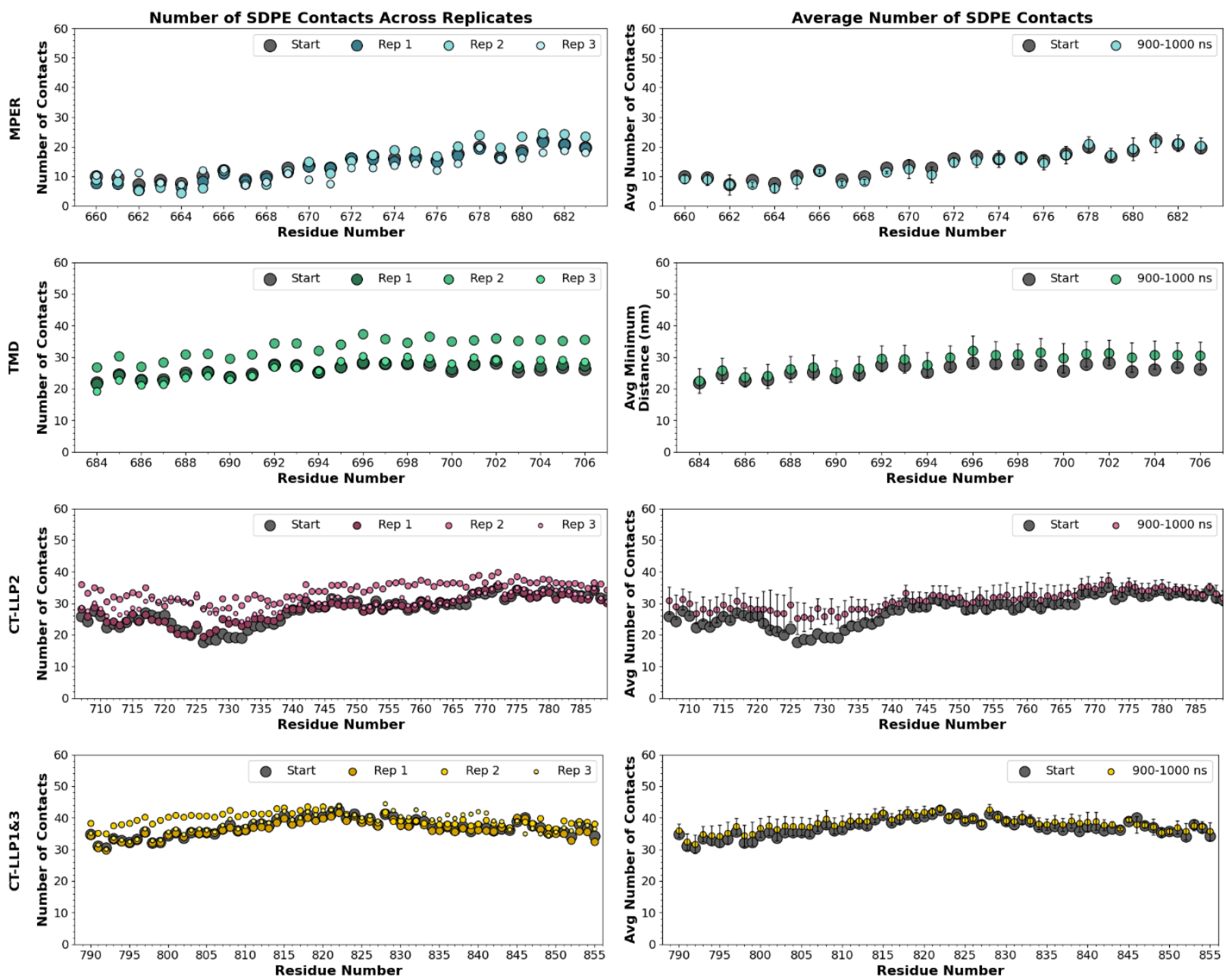

Figure S31. Normalized count of residue-SDPE interactions over the last $100 \mathrm{~ns}$ of simulation time. Contacts were normalized by dividing by the total number of cholesterol lipids in the system. Start distance and individual replicate data is shown (left) with average \pm standard deviation across three replicates is also shown (right). Each graph is colored based on the MPER-TMD-CT trimer domain (cyan - MPER, green - TMD, pink - CT ${ }^{\text {LP2 }}$, yellow - CT ${ }^{\text {LP3-LLP1 }}$ ). 

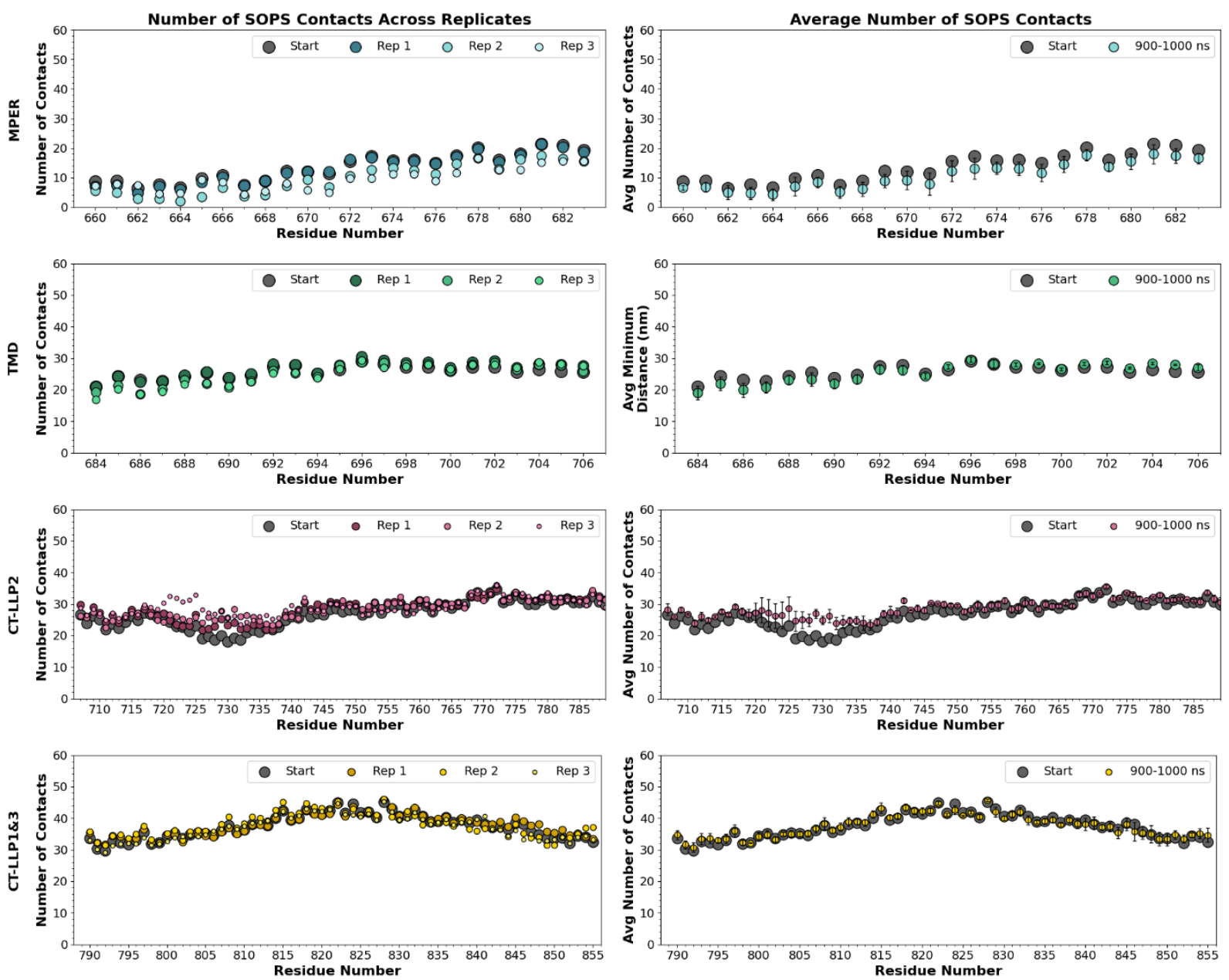

Figure S32. Normalized count of residue-SOPS interactions over the last $100 \mathrm{~ns}$ of simulation time. Contacts were normalized by dividing by the total number of cholesterol lipids in the system. Start distance and individual replicate data is shown (left) with average \pm standard deviation across three replicates is also shown (right). Each graph is

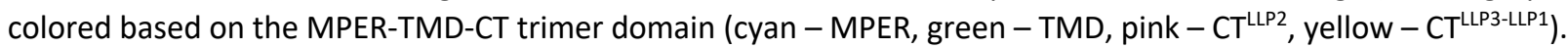



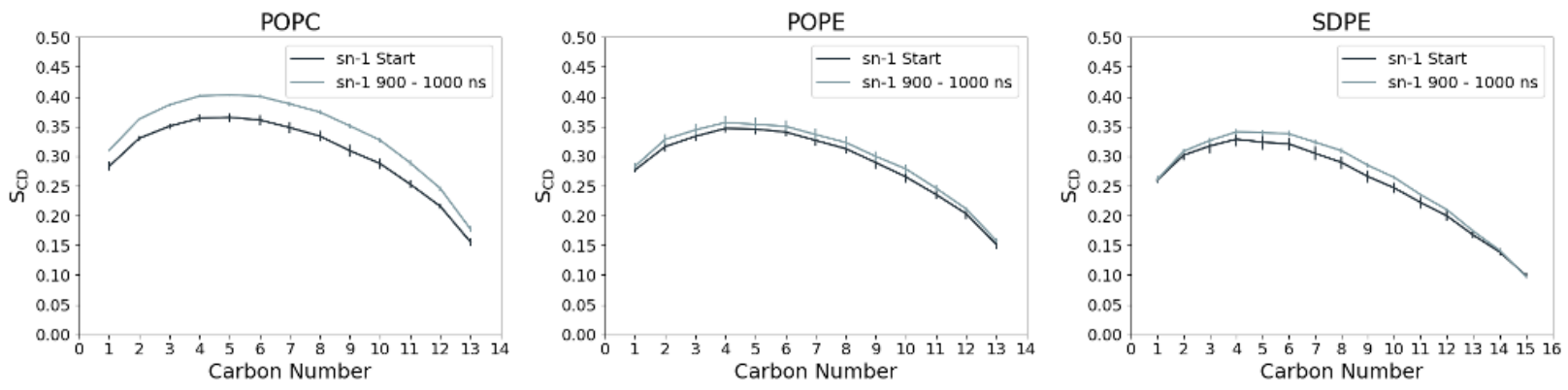

Figure S33. Deuterium order parameters on the sn-1 chain of POPC, POPE, and SDPE. Order parameters were calculated for each acyl tail carbon atom for the first $20 \mathrm{~ns}$ (start) and as an average over the last $100 \mathrm{~ns}$ of simulation. Parameters were also analyzed on the sn- 2 chain and showed similar trends. Average over replicates and standard deviation is shown. 

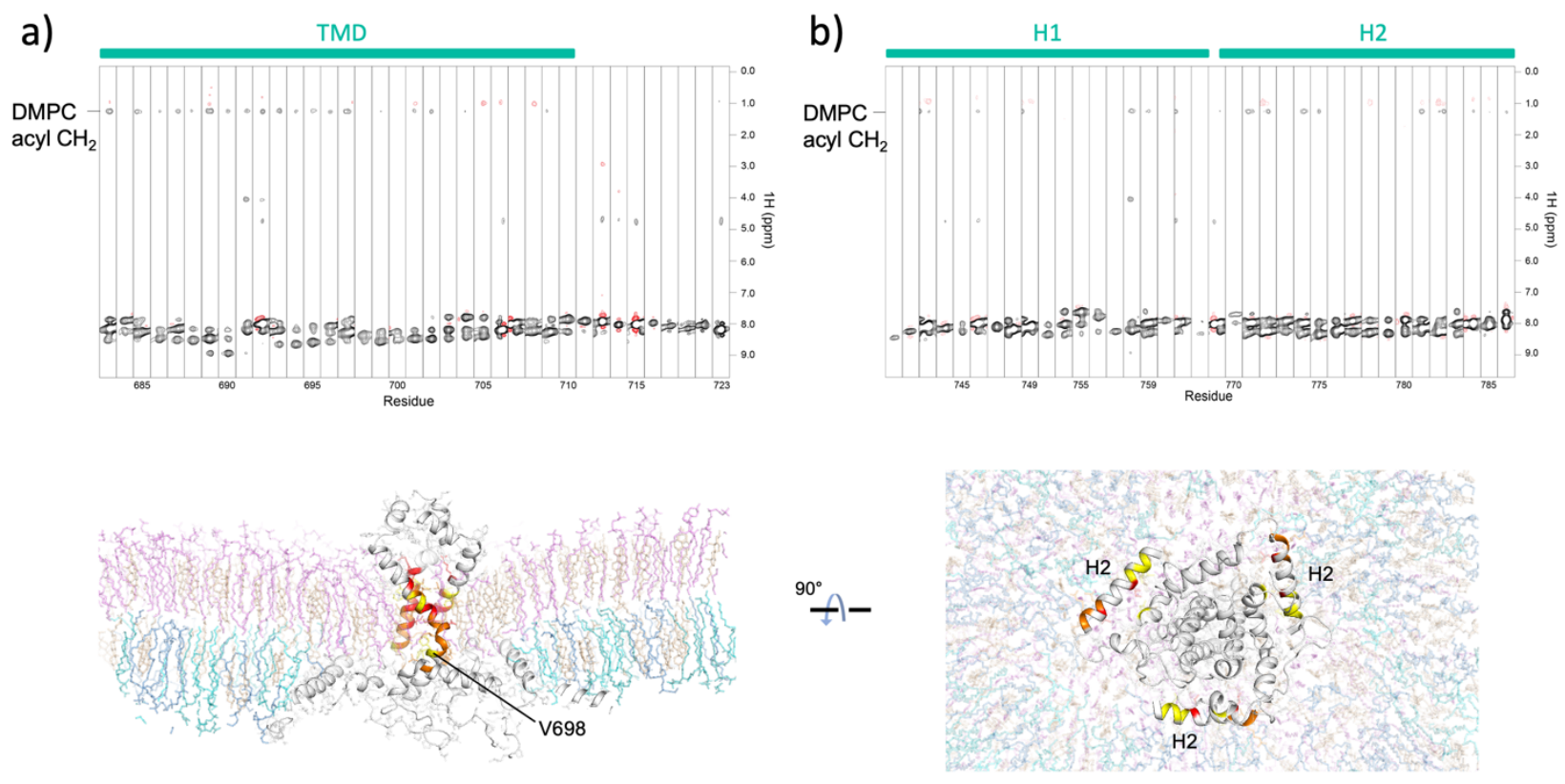

Figure S34. Protein-lipid NOE analysis for examining lipid exclusion by the CT baseplate. A 3D $J_{\mathrm{CH}}-$ modulated NOE experiment ${ }^{2}$ was recorded using a $\left({ }^{15} \mathrm{~N},{ }^{13} \mathrm{C}, 85 \%{ }^{2} \mathrm{H}\right.$ )-labeled TMD-CT ${ }^{\text {LLP2 }}$ sample reconstituted in $q=0.5$ bicelles (see Table S2 for the NMR acquisition parameters). The $J_{\mathrm{CH}^{-}}$-modulated NOE experiment is based on the regular $3 \mathrm{D}{ }^{15} \mathrm{~N}$ edited NOESY-TROSY-HSQC, in which the ${ }^{1} \mathrm{H}$ evolution period before the NOE mixing is changed to a 'mixed constanttime' evolution to introduce $8 \mathrm{~ms}$ of ${ }^{1} \mathrm{H}-{ }^{13} \mathrm{C} J$ evolutions. Consequently, the NOE peaks from the residual aliphatic protons of the $\left({ }^{15} \mathrm{~N},{ }^{13} \mathrm{C}, 85 \%{ }^{2} \mathrm{H}\right.$ )-labeled protein are negative (red) and those from the lipids are positive (black). (a) Stripes from the 3D NOE spectrum for the TMD showing residue-specific lipid NOEs (top); the NOEs are mapped onto the structure after 1000 ns of MD simulation (bottom) with NOE intensities coded by red (strong), orange (medium), and yellow (weak). (b) The same as in (a) but for the $\mathrm{H} 1$ and $\mathrm{H} 2$ helices of the LLP2 domain. 


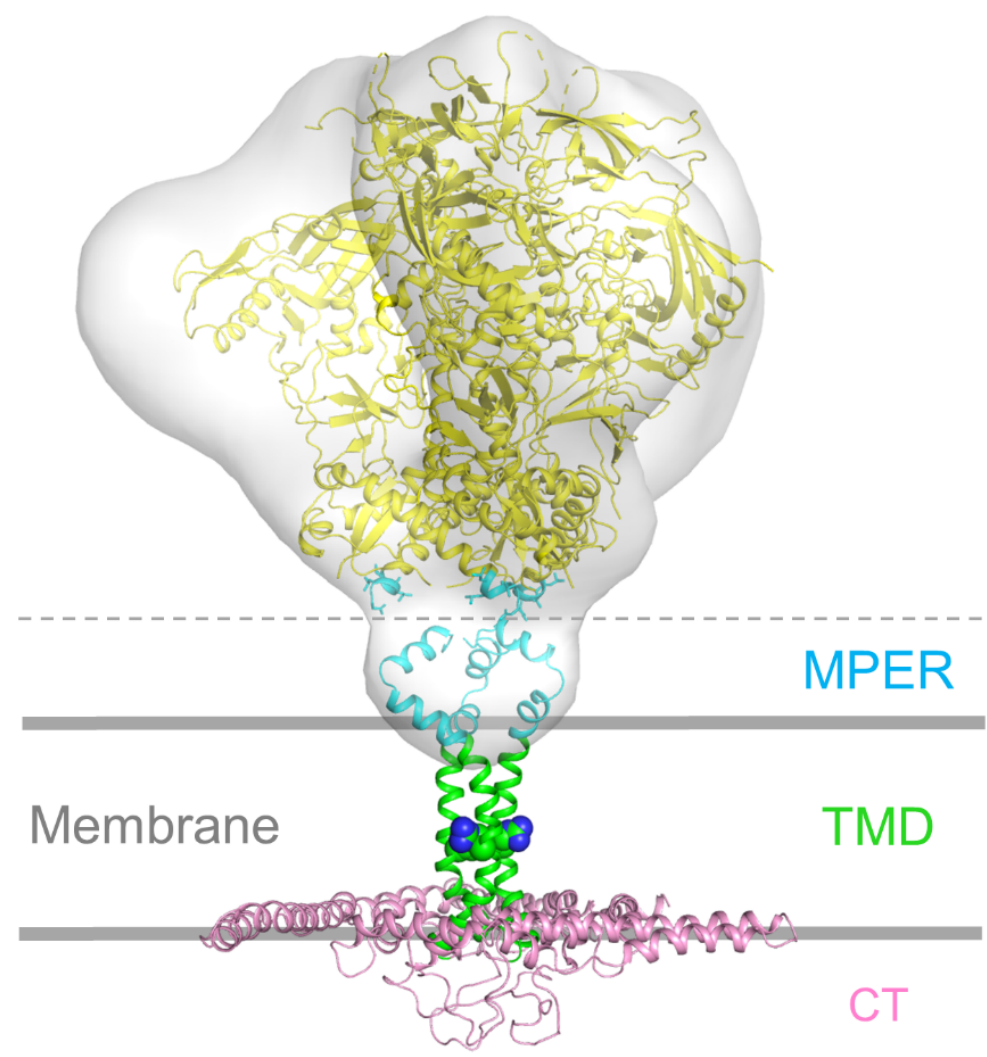

Figure S35. Fit of the trimeric MPER-TMD-CT NMR structure to the EM density of the HIV-1 Env trimer [EMDB ID: EMD-5019; 22 Å resolution] on the surface of virion derived from cryo- $\mathrm{ET}^{30}$. 


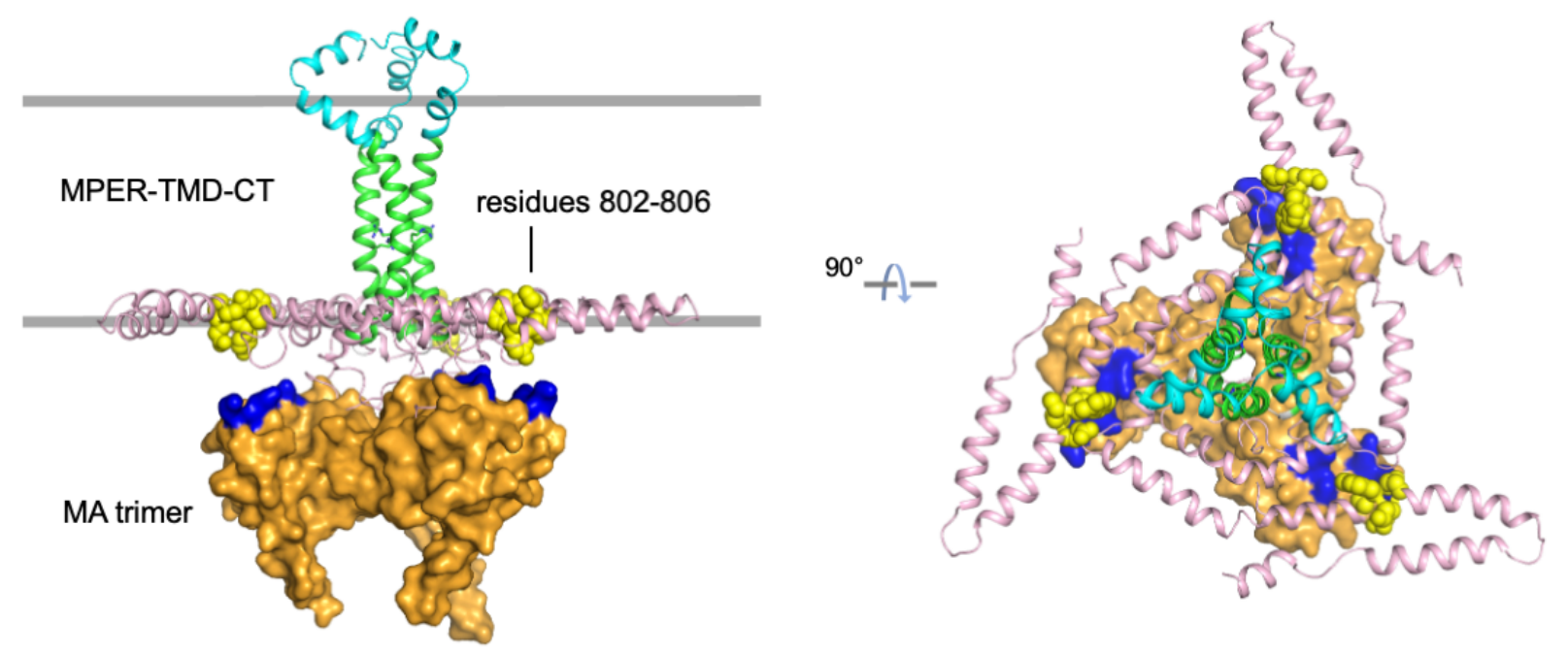

Figures S36. Evaluation of possible interaction between the Env CT and the matrix domain (MA) of the Gag polyprotein in the context of known NMR and crystal structures. Residues 802-806 of the Env CT (Y802, W803, S804, Q805, E806), shown as yellow spheres, have been suggested by previous genetic studies to be involved in the interaction between Env CT and Gag MA ${ }^{31}$. The basic residues of Gag MA (K26, K27, K30, K32), highlighted in blue, have been shown to significantly affect viral assembly ${ }^{32}$. The crystal structure of MA (PDB ID: 1 HIW; orange surface representation) ${ }^{33}$ is placed against the NMR structure of the CT baseplate (pink) such that residues $802-806$ of the $\mathrm{CT}$ and basic residues of the MA in the two trimeric complexes are aligned. The modeling suggests that a direct interaction between Env CT and Gag MA is structurally plausible. 


\section{References}

1. Piai, A.; Fu, Q.; Cai, Y.; Ghantous, F.; Xiao, T.; Shaik, M. M.; Peng, H.; Rits-Volloch, S.; Chen, W.; Seaman, M. S.; Chen, B.; Chou, J. J., Structural basis of transmembrane coupling of the HIV-1 envelope glycoprotein. Nat Commun 2020, 11 (1), 2317.

2. Fu, Q.; Piai, A.; Chen, W.; Xia, K.; Chou, J. J., Structure determination protocol for transmembrane domain oligomers. Nat Protoc 2019, 14 (8), 2483-2520.

3. Delaglio, F.; Grzesiek, S.; Vuister, G. W.; Zhu, G.; Pfeifer, J.; Bax, A., NMRPipe: A multidimensional spectral processing system based on UNIX pipes. Journal of Biomolecular NMR 1995, 6 (3), 277-293.

4. Bartels, C.; Xia, T. H.; Billeter, M.; Guntert, P.; Wuthrich, K., The program XEASY for computer-supported NMR spectral analysis of biological macromolecules. J Biomol NMR 1995, 6 (1), 1-10.

5. Dev, J.; Park, D.; Fu, Q.; Chen, J.; Ha, H. J.; Ghantous, F.; Herrmann, T.; Chang, W.; Liu, Z.; Frey, G.; Seaman, M. S.; Chen, B.; Chou, J. J., Structural basis for membrane anchoring of HIV-1 envelope spike. Science 2016, 353 (6295), 172-175.

6. Fu, Q.; Shaik, M. M.; Cai, Y.; Ghantous, F.; Piai, A.; Peng, H.; Rits-Volloch, S.; Liu, Z.; Harrison, S. C.; Seaman, M. S.; Chen, B.; Chou, J. J., Structure of the membrane proximal external region of HIV-1 envelope glycoprotein. Proc Natl Acad Sci U S A 2018, 115 (38), E8892-E8899.

7. Kay, L. E.; Ikura, M.; Tschudin, R.; Bax, A., Three-dimensional triple-resonance NMR Spectroscopy of isotopically enriched proteins. 1990. J Magn Reson 1990, 213 (2), 423-41.

8. Salzmann, M.; Wider, G.; Pervushin, K.; Senn, H.; Wuthrich, K., TROSY-type triple-resonance experiments for sequential NMR assignments of large proteins. Journal of the American Chemical Society 1999, 121 (4), $844-848$.

9. Piai, A.; Fu, Q.; Dev, J.; Chou, J. J., Optimal Bicelle Size q for Solution NMR Studies of the Protein Transmembrane Partition. Chemistry 2017, 23 (6), 1361-1367.

10. Piai, A.; Dev, J.; Fu, Q.; Chou, J. J., Stability and Water Accessibility of the Trimeric Membrane Anchors of the HIV-1 Envelope Spikes. J Am Chem Soc 2017, 139 (51), 18432-18435.

11. Schwieters, C. D.; Kuszewski, J.; Tjandra, N.; Clore, G. M., The Xplor-NIH NMR molecular structure determination package. J. Magn. Reson. 2002, 160, 66-74.

12. Shen, Y.; Delaglio, F.; Cornilescu, G.; Bax, A., TALOS+: a hybrid method for predicting protein backbone torsion angles from NMR chemical shifts. Journal of Biomolecular NMR 2009, 44 (4), 213-23.

13. Lee, J.; Cheng, X.; Swails, J. M.; Yeom, M. S.; Eastman, P. K.; Lemkul, J. A.; Wei, S.; Buckner, J.; Jeong, J. C.; Qi, Y.; Jo, S.; Pande, V. S.; Case, D. A.; Brooks, C. L., 3rd; MacKerell, A. D., Jr.; Klauda, J. B.; Im, W., CHARMM-GUI Input Generator for NAMD, GROMACS, AMBER, OpenMM, and CHARMM/OpenMM Simulations Using the CHARMM36 Additive Force Field. J Chem Theory Comput 2016, 12 (1), 405-13.

14. Markidis, S.; Laure, E., Solving Software Challenges for Exascale : International Conference on Exascale Applications and Software, EASC 2014, Stockholm, Sweden, April 2-3, 2014, Revised Selected Papers. In Theoretical Computer Science and General Issues 8759 [Online] 1st ed.; Springer International Publishing : Imprint: Springer,: Cham, 2015; pp. 1 online resource (VIII, 149 pages 61 illustrations).

15. Abraham, M. J.; Murtola, T.; Schulz, R.; Pall, S.; Smith, J. C.; Hess, B.; Lindahl, E., GROMACS: High performance molecular simulations through multi-level parallelism from laptops to supercomputers. SoftwareX 2015, 1-2, 19-25.

16. Hollingsworth, L. R. t.; Lemkul, J. A.; Bevan, D. R.; Brown, A. M., HIV-1 Env gp41 Transmembrane Domain Dynamics Are Modulated by Lipid, Water, and Ion Interactions. Biophys J 2018, 115 (1), 84-94.

17. Brugger, B.; Glass, B.; Haberkant, P.; Leibrecht, I.; Wieland, F. T.; Krausslich, H. G., The HIV lipidome: a raft with an unusual composition. Proc Natl Acad Sci U S A 2006, 103 (8), 2641-6.

18. Huang, J.; Rauscher, S.; Nawrocki, G.; Ran, T.; Feig, M.; de Groot, B. L.; Grubmuller, H.; MacKerell, A. D., Jr., CHARMM36m: an improved force field for folded and intrinsically disordered proteins. Nat Methods 2017, 14 (1), 71-73.

19. Jorgensen, W. L.; Chandrasekhar, J.; Madura, J. D.; Impey, R. W.; Klein, M. L., Comparison of simple potential functions for simulating liquid water. J. Chem. Phys 1983, 79 (2), 926-935.

20. Luo, Y.; Roux, B., Simulation of Osmotic Pressure in Concentrated Aqueous Salt Solutions. J. Phys. Chem. Lett. 2010, 1 (1), 183-189.

21. Berendsen, H. J. C.; Postma, J. P. M.; van Gunsteren, W. F.; DiNola, A.; Haak, J. R., Molecular dynamics with coupling to an external bath. J. Chem. Phys. 1984, 81. 
22. Hoover, W. G., Canonical dynamics: Equilibrium phase-space distributions. Phys Rev A Gen Phys 1985, 31 (3), 1695-1697.

23. Nose, S.; Klein, M. L., Constant pressure molecular dynamics for molecular systems. Mol. Phys 1983, 50.

24. Parrinello, M.; Rahman, A., Polymorphic transitions in single crystals: A new molecular dynamics method. J. Appl. Phys 1981, 52 (12), 7182-7190.

25. Hess, B., P-LINCS: A Parallel Linear Constraint Solver for Molecular Simulation. J Chem Theory Comput 2008, 4 (1), 116-22.

26. Darden, T.; York, D.; Pedersen, L., Particle mesh Ewald: An N·log(N) method for Ewald sums in large systems. J. Chem. Phys 1993, 98.

27. Essmann, U.; Perera, L.; Berkowitz, M. L., A smooth particle mesh Ewald method. J. Chem. Phys 1995, 103.

28. Crooks, G. E.; Hon, G.; Chandonia, J. M.; Brenner, S. E., WebLogo: a sequence logo generator. Genome Res 2004, $14(6), 1188-90$.

29. Buchoux, S., FATSLiM: a fast and robust software to analyze MD simulations of membranes. Bioinformatics 2017, 33 (1), 133-134.

30. Liu, J.; Bartesaghi, A.; Borgnia, M. J.; Sapiro, G.; Subramaniam, S., Molecular architecture of native HIV-1 gp120 trimers. Nature 2008, 455 (7209), 109-13.

31. Murakami, T.; Freed, E. O., Genetic evidence for an interaction between human immunodeficiency virus type 1 matrix and alpha-helix 2 of the gp41 cytoplasmic tail. $J$ Virol 2000, 74 (8), 3548-54.

32. Freed, E. O.; Englund, G.; Martin, M. A., Role of the basic domain of human immunodeficiency virus type 1 matrix in macrophage infection. J Virol 1995, 69 (6), 3949-54.

33. Hill, C. P.; Worthylake, D.; Bancroft, D. P.; Christensen, A. M.; Sundquist, W. I., Crystal structures of the trimeric human immunodeficiency virus type 1 matrix protein: implications for membrane association and assembly. Proc Natl Acad Sci U S A 1996, 93 (7), 3099-104. 